\title{
WestVirginiaUniversity
}

THE RESEARCH REPOSITORY @ WVU

Graduate Theses, Dissertations, and Problem Reports

2014

\section{Group III-nitride devices and applications}

Joshua L. Justice

West Virginia University

Follow this and additional works at: https://researchrepository.wvu.edu/etd

\section{Recommended Citation}

Justice, Joshua L., "Group III-nitride devices and applications" (2014). Graduate Theses, Dissertations, and Problem Reports. 465.

https://researchrepository.wvu.edu/etd/465

This Thesis is protected by copyright and/or related rights. It has been brought to you by the The Research Repository @ WVU with permission from the rights-holder(s). You are free to use this Thesis in any way that is permitted by the copyright and related rights legislation that applies to your use. For other uses you must obtain permission from the rights-holder(s) directly, unless additional rights are indicated by a Creative Commons license in the record and/ or on the work itself. This Thesis has been accepted for inclusion in WVU Graduate Theses, Dissertations, and Problem Reports collection by an authorized administrator of The Research Repository @ WVU. For more information, please contact researchrepository@mail.wvu.edu. 


\title{
GROUP III-NITRIDE DEVICES AND APPLICATIONS
}

by

\author{
Joshua L. Justice
}

BSEE and BSCpE

A thesis submitted to the

\section{Benjamin M. Statler College of Engineering and Mineral Resources \\ at \\ WEST VIRGINIA UNIVERSITY}

in partial fulfillment for the degree of

Master of Science

in

\section{Electrical Engineering}

Committee members:

Dr. Dimitris Korakakis, Committee Chairperson

Dr. Lawrence A. Hornak

Dr. Jeremy M. Dawson

Lane Department of Computer Science and Electrical Engineering Morgantown, WV 26506

Keywords: Group III-Nitrides, Surface Acoustic Wave Devices, High Electron Mobility Transistors, Metalorganic Chemical Vapor Deposition

April 7, 2014

(C)2014 Joshua L. Justice 



\title{
ABSTRACT
}

\section{Group III-Nitride Devices and Applications}

\author{
Joshua L. Justice
}

The group III-nitride system of materials has had considerable commercial success in recent years in the solid state lighting (SSL) and power electronics markets. The need for high efficient general lighting applications has driven research into InGaN based blue light emitting diodes (LEDs), and demand for more efficient power electronics for telecommunications has driven research into AlGaN based high electron mobility transistors (HEMTs). However, the group III-nitrides material properties make them attractive for several other applications that have not received as much attention. This work focuses on developing group III-nitride based devices for novel applications.

$\mathrm{GaN}$ is a robust, chemically inert, piezoelectric material, making it an ideal candidate for surface acoustic wave (SAW) devices designed for high temperature and/or harsh environment sensors. In this work, SAW devices based on GaN are developed for use in high temperature gas or chemical sensor applications. To increase device sensitivity, while maintaining a simple one-step photolithography fabrication process, devices were designed to operate at high harmonic frequencies. This allows for $\mathrm{GHz}$ regime operation without sub-micron fabrication. One potential market for this technology is continuous emissions monitoring of combustion gas vehicles.

In addition to SAW devices, high electron mobility transistors (HEMTs) were developed. The epitaxial structure was characterized and the 2-D electron gas concentrations were simulated and compared to experimental results. Device fabrication processes were developed and are outlined. Fabricated devices were electrically measured and device performance is discussed. 



\section{Acknowledgements}

Above all, I would like to thank my wife, Jackie, whose patience, loving support, and commitment to our family allowed me to pursue this degree. She deserves as much, if not more credit for her willingness to leave all her family and friends behind in San Diego, move to Morgantown, WV with me, and raise our son while I attended school.

I would like to thank my son, Joshua, who even at a young age, showed interest and enthusiasm in my work. His questions helped me think about this work in ways I would not have otherwise.

I would like to thank my advisor, Dr. Dimitris Korakakis, for taking the chance, and giving me the opportunity to conduct this research. His time, discussions, guidance and mentoring were invaluable. Not only for progressing this work, but for helping me establish a fundamental skill set and a confidence in my abilities that will allow me to be successful in my career far beyond grad school.

I would like to thank the committee members, Dr. Larry Hornak and Dr. Jeremy Dawson. Both of whom made themselves available and were always willing to help when I had questions. Specifically, Dr. Hornak's help in device design to suppress the electromagnetic feedthrough of the SAW devices and Dr. Dawson's help in optimizing the fabrication processes in the cleanroom.

I would like to thank the following WVU faculty for their valuable discussion and insight into this work: Dr. Osama Mukdadi, Dr. David Graham, Dr. Charter Stinespring, Dr. Nick Wu, Dr. Mark Jerabek, Dr. Natalia Schmid, Dr. Yuxin Liu and Dr. Alan Bristow.

I would like to thank the following WVU Shared Research Facilities members for all the time they spent helping me with the equipment: Dr. Kolin Brown, Dr. Weiqiang Ding and Harley Hart.

Last of all, but certainly not least, I would like to thank all my labmates. Without their help, I never would have been able to get anything accomplished. Anand Kadiyala, Vishal Narang, Vamsi Kumbham, Dr. Sridhar Kuchibhatla, Justin Peacock, Kevin Oresick, Kenneth Hite, Dr. Kyoungnae Lee, Dr. Lee Rodak, Dr. Ronak Rahimi, Bashar Hamza, Srinitya Musunuru, Lekha Kuchipudi, and Sujan Kasani. 



\section{Table of Contents}

List of Figures $\quad$ ix

List of Tables $\quad$ xi

List of Symbols $\quad$ xiii

List of Acronyms $\quad$ Xv

Chapter 1 Introduction 1

1.1 Statement of the Problem and Scope of Work . . . . . . . . . . . . . 1

1.2 Outline . . . . . . . . . . . . . . . . . . 2

Chapter 2 Background 5

2.1 Review of Surface Acoustic Wave Devices . . . . . . . . . . . . . . . 5

2.2 Review of Group III-Nitride Materials . . . . . . . . . . . . . . 8

Chapter 3 Materials and Devices $\quad 11$

3.1 Group III-Nitride Materials Growth . . . . . . . . . . . . . . . . . . . . 11

3.1 .1 Metal Organic Vapor Phase Epitaxy . . . . . . . . . . . . . . . 12

3.1.2 GaN for SAW Devices . . . . . . . . . . . . . . . . . 13

3.1.3 $\mathrm{Al}_{x} \mathrm{Ga}_{1-x} \mathrm{~N} / \mathrm{GaN}$ Heterojunctions for HEMTs . . . . . . . . . 13

3.2 Device Fabrication . . . . . . . . . . . . . . . . . . 16

3.2.1 SAW Device Fabrication . . . . . . . . . . . . . . . . 17

3.2 .2 HEMT Fabrication . . . . . . . . . . . . . . . . . . 17

3.3 Electro-Acoustic Characterization . . . . . . . . . . . . . . . 22

Chapter 4 Surface Acoustic Wave Devices 25

4.1 Introduction . . . . . . . . . . . . . . . . . 25

4.1.1 Interdigital Transducer Design . . . . . . . . . . . . . . . 25

4.1.2 SAW Devices as Chemical Sensors . . . . . . . . . . . . . . . 28

4.2 GaN Based Surface Acoustic Wave Devices . . . . . . . . . . . . . . . . . 29

4.2.1 Frequency Response of SAW Devices on GaN . . . . . . . . . . . 30

4.2.2 Non-Rayleigh SAW Modes in GaN . . . . . . . . . . . . . . . . . 31

4.3 Harmonic SAW Devices . . . . . . . . . . . . . . . . . . . 33

4.3.1 Harmonic SAW Device Design . . . . . . . . . . . . . . 33

4.3.2 Harmonic SAW Device Frequency Response . . . . . . . . . . . . . 35

4.4 Substrate Effects on Propagation of SAW in GaN . . . . . . . . . . . . . 38

4.5 GaN Based SAW Devices for High Temperature Applications . . . . . . . 40

4.6 GaN Based SAW Devices for Continous Emissions Monitoring . . . . . . 44

4.7 Electromagnetic Feedthrough . . . . . . . . . . . . . 50 
Chapter 5 High Electron Mobility Transistors 53

5.1 Introduction . . . . . . . . . . . . . . . . . 53

5.2 Theoretical Results . . . . . . . . . . . . . . . . . 57

5.3 Experimental Results . . . . . . . . . . . . . . . 63

$\begin{array}{lll}\text { Chapter } 6 & \text { Conclusions } & 65\end{array}$

Appendix A Common Second Order SAW Effects and Design Solutions $\quad 71$

$\begin{array}{lll}\text { Appendix B SAW Device Fabrication Procedures } & 73\end{array}$

$\begin{array}{lll}\text { Appendix C HEMT Fabrication Procedures } & 75\end{array}$

$\begin{array}{lll}\text { Appendix D VNA Calibration and Operation } & 79\end{array}$

$\begin{array}{ll}\text { Bibliography } & 81\end{array}$ 


\section{List of Figures}

2.1 Group III-Nitride Family Bandgaps and Lattice Constants . . . . . . . . 9

3.1 Picture of the MOVPE System . . . . . . . . . . . . . . . 11

3.2 Schematic of HEMT Structure . . . . . . . . . . . . . . . . . . . . . . 14

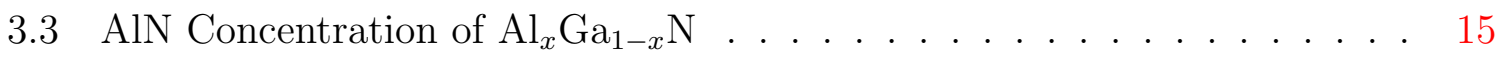

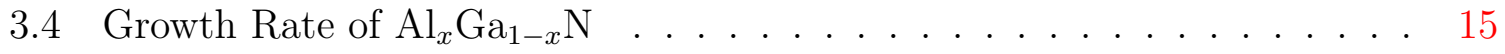

3.5 ICP Etch Rate of $\mathrm{Al}_{x} \mathrm{Ga}_{1-x} \mathrm{~N} \ldots \ldots \ldots$

3.6 Specific Contact Resistance From TLM Measurement . . . . . . . . . . . 20

3.7 HEMT Contact Optimization . . . . . . . . . . . . . . . . . . 21

3.8 Optical Image of Fabricated HEMT Device . . . . . . . . . . . . . . . . . 21

4.1 Basic Geomerty of an Interdigital Transducer . . . . . . . . . . . . 26

4.2 SAW Device as a Sensor . . . . . . . . . . . . . . . . 28

4.3 Schematic Showing Cross Sectional View of SAW in GaN Thin Film . . . 29

4.4 Comparison of Rayleigh Mode SAW on Different Materials . . . . . . . . 30

4.5 Non-Rayliegh Mode SAWs in GaN Thin Films . . . . . . . . . . . . . . . 32

4.6 Harmonic SAW Device Design . . . . . . . . . . . . . . . . . . . . 34

4.7 Harmonic SAW Device Wideband Frequency Response . . . . . . . . . . 36

4.8 Harmonic SAW Devices, Local Frequency Response Comparison . . . . . 37

4.9 SAW Dispersion in GaN Thin Films . . . . . . . . . . . . . . . 38

4.10 Substrate Effect on SAWs Travelling in GaN Thin Films . . . . . . . . . 39

4.11 AFM on Thermally Cycled GaN . . . . . . . . . . . . . . . . . . 41

4.12 EDS Measurements of Thermally Cycled GaN . . . . . . . . . . . . . . . 42

4.13 Frequency Response of Thermally Cycled GaN SAW Device . . . . . . . 43

4.14 Thermal Cycling Effects on SAW Device Performance . . . . . . . . . . . 44

4.15 Real World Exhaust Engine Test Setup . . . . . . . . . . . . . . . . . . . 46

4.16 Sample Mounting for Real World Exhaust Test . . . . . . . . . . . . . . 47

4.17 SAW for CEM - Typical Engine RPM Profile . . . . . . . . . . . . . . . 47

4.18 Local Response of 7th Harmonic Device Used in CEM Testing . . . . . . 48

4.19 Real World Exhaust Testing of GaN SAW Devices . . . . . . . . . . . . . 49

4.20 Comparison of Two Ground Electrode Gemoetries . . . . . . . . . . . . . 51

4.21 Harmonic SAW Responses with Different Ground Electrode Geometries . 51

5.1 Schematic of Modulation Doped Superlattice . . . . . . . . . . . . . 53

5.2 Carrier Densities in the Mod-Doped GaAs/ $\mathrm{Al}_{x} \mathrm{Ga}_{1-x} \mathrm{As}$ Heterostructure . 55

5.3 Conduction Band Edge for the nAlGaN/GaN MODFET Structure . . . . 56

5.4 Polarization Fields in AlGaN / GaN Heterojunction . . . . . . . . . . . . 58

5.5 Calculated Polarization Sheet Charge Density in strained $\mathrm{Al}_{x} \mathrm{Ga}_{1-x} \mathrm{~N}$. . 60

5.6 Calculated Polarization Induced Sheet Charge Concentration in strained $\mathrm{Al}_{x} \mathrm{Ga}_{1-x} \mathrm{~N} \ldots \ldots \ldots \ldots \ldots$. . . . . . . . . . . . . . . . . . . 61 
5.7 HEMT 2DEG Mobility . . . . . . . . . . . . . . . 61

5.8 Experimental Results for 2DEG Sheet Charge Density . . . . . . . . . 62

5.9 HEMT Drain Sweep . . . . . . . . . . . . . . . . . . 63

5.10 HEMT Gate Sweep . . . . . . . . . . . . . . . . . . . . . 64 


\section{List of Tables}

2.1 Surface Acoustic Wave Device Applications . . . . . . . . . . . . . . 7

4.1 Design Parameters of Harmonic SAW Devices . . . . . . . . . . . . . 35

4.2 SAW for CEM - Engine Exhaust Test Parameters . . . . . . . . . . . . . 47 



\section{List of Symbols}

$\begin{array}{ll}f & \text { Frequency } \\ f_{0} & \text { Characteristic Frequency } \\ f_{O p} & \text { Operating Frequency } \\ \lambda & \text { Wavelength } \\ \lambda_{0} & \text { Characteristic Wavelength } \\ \lambda_{e f f} & \text { Effective Wavelength } \\ \eta & \text { Metalization Ratio } \\ S_{i j} & \text { Scattering Parameter } \\ \left|S_{21}\right| & \text { Magnitude of } S_{21} \text { measured in dB } \\ \nu & \text { Acoustic Velocity } \\ \nu_{P} & \text { Poisson's Ratio }\end{array}$





\section{List of Acronyms}

ACP

AFM

AlN

$\mathrm{Al}_{x} \mathrm{Ga}_{1-x} \mathrm{~N}$

BAW

BOE

$\mathrm{CP}_{2} \mathrm{Mg}$

$\mathrm{dB}$

DBR

DC

DUT

EDS

EL

ELOG

FWHM

GaN

HEMT

HIDT

HVPE

IDT

$\mathrm{In}_{x} \mathrm{Ga}_{1-x} \mathrm{~N}$

IR

ISS

ITO

IV

LED

MBE

MEMS

MFC

MOCVD

MOVPE
Air Coplanar

Atomic Force Microscopy

Aluminum Nitride

Aluminum Gallium Nitride

Bulk Acoustic Wave

Buffered Oxide Etchant

Bis-cyclopentadienyl Magnesium

Decibels

Distributed Bragg Reflector

Direct Current

Device Under Test

Energy Dispersive X-Ray Spectroscopy

Electroluminescence

Epitaxial Lateral Overgrowth

Full Width Half Maximum

Gallium Nitride

High Electron Mobility Transistor

Harmonic Interdigital Transducer

Hydride Vapor Phase Epitaxy

Interdigitated Transducer or Interdigital Transducer

Indium Gallium Nitride

Infrared

Impedance Standard Substrate

Indium Tin Oxide

Current - Voltage

Light Emitting Diode

Molecular Beam Epitaxy

Microelctromechanical System

Mass Flow Controller

Metal Organic Chemical Vapor Phase Epitaxy

Metal Organic Vapor Phase Epitaxy 


$\begin{array}{ll}\text { MQW } & \text { Mulitple Quantum Well } \\ \text { MSMM } & \text { Multi-sensor Manipulation Module } \\ \text { MSC } & \text { Multi-strip Coupler } \\ \mathrm{NH}_{3} & \text { Ammonia } \\ \text { PC } & \text { Pressure Controller } \\ \text { PECVD } & \text { Plasma Enhanced Chemical Vapor Deposition } \\ \text { PNA } & \text { Programmable Network Analyzer } \\ \text { QCM } & \text { Quartz Crystal Microbalance } \\ \text { RF } & \text { Radio Frequency } \\ \text { RS } & \text { Reactive Sputtering } \\ \text { SAG } & \text { Selective Area Growth } \\ \text { SAW } & \text { Surface Acoustic Wave } \\ \text { SCCM } & \text { Standard Cubic Centimeters per Minute } \\ \text { SEM } & \text { Scanning Electron Microscopy } \\ \text { SPR } & \text { Surface Plasmon Resonance } \\ \text { TEGa } & \text { Triethylgallium } \\ \text { TMAl } & \text { Trimethylaluminum } \\ \text { TMGa } & \text { Trimethylgallium } \\ \text { TMIn } & \text { Trimethlyindium } \\ \text { UV } & \text { Ultra-Violet } \\ \text { wrt } & \text { with respect to } \\ \text { WVU } & \text { West Virginia University } \\ \text { XRD } & \text { X-Ray Diffraction }\end{array}$




\section{Chapter 1}

\section{Introduction}

\subsection{Statement of the Problem and Scope of Work}

The group III-nitride (III-N) family of materials consists of boron nitride (BN), aluminum nitride $(\mathrm{AlN})$, gallium nitride $(\mathrm{GaN})$, indium nitride $(\mathrm{InN})$ and thallium(I) nitride $\left(\mathrm{Tl}_{3} \mathrm{~N}\right)$. BN has many useful commercial applications but is not generally used as a semiconductor material. $\mathrm{Tl}_{3} \mathrm{~N}$ nitride is a very unstable compound and is not discussed further. For the purposes of this work, and most works dealing with semiconductor materials, the term group III-nitrides refers only to $\mathrm{AlN}, \mathrm{GaN}, \mathrm{InN}$, and their alloys. Even more generally, the term GaN-based is often used, and includes AlN and InN.

The III-N's have become one of the most successful and promising material systems for a variety of applications including light emitting diodes (LEDs) and high electron mobility transistors (HEMTs). Despite early shortcomings in efficiency and brightness, very high brightness and high efficiency blue LEDs are currently being manufactured for use in white light LEDs. These white LEDs will most likely replace all traditional lighting including incandescents and fluorescents. The transition to solidstate lighting via GaN LEDs will result in considerable energy cost savings. Thanks in part to the large power density of GaN, GaN based HEMTs have already surpassed silicon, silicon carbide, and GaAs transistors in efficiency and speed. GaN transistors will also most likely replace existing semiconductor technology in power and high speed applications include telecommunications, radar and satellite applications. While these technologies are being vigorously researched and developed, other GaN material properties, such as its piezoelectricity, chemical inertness, low toxicity, bio-compatibility and 
radiation hardness make it an attractive material for a multitude of other niche markets. These markets include surface acoustic wave (SAW) devices for biological and harsh environment sensors, UV LEDs for water purification systems, UV (aka solar-blind ) photodiodes (UV-PDs) for space applications and as a base for integrating electronic and optoelectronic devices made from the same materials.

Relatively little research and development has been conducted on these other applications for group III-nitrides when compared to LEDs and HEMTs. In addition, surface acoustic wave devices for telecommunications have become a multi-billion dollar industry, but SAW devices as sensors are still in their infancy. Very little work has been done to investigate GaN as a material for SAW devices. The scope of this work focuses on developing GaN based SAW devices for biological and harsh environment sensor applications. Specifically, SAW devices that operate in the GHz regime without the necessity for sub-micron fabrication are been developed. These devices are then developed for high temperature applications and gas sensor applications with the potential for being used in continuous emissions monitoring (CEM) systems.

Additionally, HEMTs have been developed and LEDs already developed by the group have been improved and expanded to operate as photodiodes. These devices are then integrated into electronic and optoelectronic chips with robotic space servicing applications in mind.

\subsection{Outline}

This work attempts to develop several GaN based devices for a few different applications. Chapter 2 gives a background into group III-nitride materials and devices. This serves as the literature review for which the bulk of the work is based and builds on.

Issues with materials growth is outside the scope of this work and are only briefly touched upon in Chapter 3. The references given provide detailed discussion on III-nitride materials growth and characterization. A detailed examination of the device 
fabrication and characterization is covered in this chapter.

Chapter 4 discuses the development of GaN based SAW devices. Device design is explained in depth and SAW device operation as a sensor is covered. Different SAW modes on GaN are described. Harmonic SAW devices are developed for $\mathrm{GHz}$ regime operation while maintaining a cheap and easy fabrication process. The effect of the sapphire substrate on SAW device performance is examined in detail. SAW devices fro high temperature and continuous emissions monitoring applications are discussed. Finally, electromagnetic feed through and its detrimental effects on SAW device performance is discussed along with design solutions.

Chapter 5 focuses on the development of the HEMT devices used in this work. Design and fabrication are covered. Theoretical 2-D electron gas concentrations are calculated and compared with experimental results.

Finally, Chapter 6 summarizes the findings and conclusions of this work. 



\section{Chapter 2}

\section{Background}

\subsection{Review of Surface Acoustic Wave Devices}

Rayleigh mode surface acoustic waves (SAWs) were first proposed by Lord Rayleigh in 1885 [1]. Rayleigh waves are generated during seismic activity in the earth and are typically the most destructive wave produced during an earthquake. The amplitude of Rayleigh waves decay as $1 / \sqrt{r}$, where $r$ is the radial distance from a point source [2]. In addition, noticeable particle displacement within a solid cause by Rayleigh waves is only measurable to approximately one acoustic wavelength into the solid. It is therefore, generally acceptable to think of Rayleigh waves as only existing within one acoustic wavelength of the surface on which they are propagating [3]. It is then easy to see that as frequency increases, and thus the wavelength decreases; the whole of the Rayleigh wave exists closer to the surface. This becomes quite substantial when designing Rayleigh wave SAW devices. Generally speaking, the higher the frequency of operation, the more sensitive the SAW device [4].

The stress-free boundary imposed by the surface of a crystal gives rise to the ability for it to allow propagation of SAWs [5]. SAW devices take advantage of the piezoelectric property of certain materials to convert an electrical potential into a mechanical strain. Because of their surface confinement, SAWs are conveniently generated by surface electrodes on a piezoelectric substrate. Many materials exhibit piezoelectricity including crystals (quartz), semiconductors (GaN), ceramics (PZT), and polymers (vinylidene polyfluoride). The simplest SAW device consists of a piezoelectric layer and a pair of IDTs. More complex arrangements are utilized for different SAW device 
applications.

In 1965, R. M. White and F. W. Voltmer were the first to successfully generate Rayleigh mode surface acoustic waves on a piezoelectric substrate using interdigitated transducers (IDTs) [6]. Since that breakthrough, there has been considerable research into SAW devices. SAW device are inherently good bandpass filters and are passive devices with no moving parts. They are also relatively cheap to produce. Thanks to these qualities, SAW devices have become ubiquitous in the telecommunications industries and have penetrated several other technological markets [7]. SAW devices can be found in smart phones, GPS systems, radar systems and satellite communications systems, to name a few. A list of SAW devices and their applications can be found in Table 2.1.

SAW devices can also be fabricated on suspended thin films or diaphragms. When a Rayleigh mode SAW is guided in a plate, it is known as a Lamb wave [9]. In addition, considerable research devoted to SAW devices for chemical and biological sensors $[4,10-15]$. When implemented as a sensor, the SAW interacts with the device surface causing measurable differences between the input and output signals. Coupling to any medium contacting the surface strongly affects the velocity and/or amplitude of the wave [4]. Because a SAW propagating in a piezoelectric medium generates mechanical deformation and an electrical potential, both mechanical and electrical coupling between the SAW and surface contacting medium are possible [5]. The three main SAW modes that are utilized in SAW sensors are Rayleigh, Lamb, and Love wave modes. Rayleigh and Lamb wave modes exhibit strong coupling with media contacting the piezoelectric surface. Thus, both Rayleigh-wave sensors are only and Lamb-wave sensors are mostly used as gas sensors, since the waves are damped in liquids [4]. Love waves are transverse waves and exhibit very poor coupling with media at the piezoelectric surface. Therefore, SAW devices are usually designed to utilize Love waves when they operate in liquid environments and it is undesirable for the liquid to attenuate the SAW. SAW devices as 
Table 2.1: Applications of SAW Devices, from [8]

\begin{tabular}{|c|c|}
\hline Type of SAW Device & Applications \\
\hline $\begin{array}{l}\text { Medium loss SAW } \\
\text { filter, (nondispersive) }\end{array}$ & $\begin{array}{l}\text { IF filtering. Clock recovery. Nyquist filters. MSK } \\
\text { modulation. }\end{array}$ \\
\hline SAW delay line & $\begin{array}{l}\text { Path length equalizers. Altimeters. Pressure and } \\
\text { temperature sensors. Tunable oscillators. Recirculating } \\
\text { storage. }\end{array}$ \\
\hline Fixed-tap delay line & $\begin{array}{l}\text { Pulse compression radar. Barkerand quadraphase coding. } \\
\text { Radar return simulation. }\end{array}$ \\
\hline $\begin{array}{l}\text { Programmable } \\
\text { transversal filter }\end{array}$ & $\begin{array}{l}\text { Adaptive filtering for spread spectrum. Matched filtering. } \\
\text { Channel equalization. Radar simulator. }\end{array}$ \\
\hline SAW Comb filter & Multiplexers. Multimode oscillators. Counters. \\
\hline Low-loss SAW filter & $\begin{array}{l}\text { VHF/UHF front-end filtering. Mobile and cellular radio. } \\
\text { Antenna duplexer. }\end{array}$ \\
\hline SAW resonator & Precision filters and fixed oscillators. \\
\hline SAW chirp filter & $\begin{array}{l}\text { Pulse compression radar. Variable delay lines and multi path } \\
\text { cancellation. Real-time Fourier transform processors. } \\
\text { Wideband linear-phase filters. Reflective array compressor } \\
\text { with large TB product. }\end{array}$ \\
\hline $\begin{array}{l}\text { Three- and four-port } \\
\text { SAW convolver }\end{array}$ & $\begin{array}{l}\text { Matched filtering in spread spectrum. Long-code correlation. } \\
\text { Radar. Packet radio. }\end{array}$ \\
\hline $\begin{array}{l}\text { Acoustic charge } \\
\text { transport on GaAs }\end{array}$ & High-speed sampling. Wide-band pn-code correlator. \\
\hline $\begin{array}{l}\text { Other SAW devices } \\
\text { on GaAs }\end{array}$ & $\begin{array}{l}\text { Programmable filtering. Tunable delay lines and resonators. } \\
\text { Programmable filters and correlators. }\end{array}$ \\
\hline $\begin{array}{l}\text { SAW-based } \\
\text { optoelectronic devices }\end{array}$ & $\begin{array}{l}\text { Bragg modulators. Spectrum analyzers. Wide-band } \\
\text { convolvers and correlators. Monolithic integration on GaAs. }\end{array}$ \\
\hline $\begin{array}{l}\text { Multilayered SAW } \\
\text { devices }\end{array}$ & $\begin{array}{l}\text { Rugged resonators and filters. Monolithic SAW convolvers } \\
\text { and correlators. Sensors. }\end{array}$ \\
\hline
\end{tabular}


chemical sensors are explained more in section 4.1.2.

SAW devices are typically made up of two adjacent interdigital transducers (IDTs). This configuration is known as a delay-line type SAW device because acoustic waves travel much slower then electromagnetic waves. SAW delay lines simply retain signal information for a period of time similar to a buffer in an integrated circuit. Because acoustic waves travels considerably slower than signals in a digital circuit, information is temporarily stored and becomes available after an amount of time determined by the length of the delay line. A typical SAW delay line of $1 \mathrm{~cm}$ can store 2 to $3 \mathrm{~ms}$ of information [7]. Delay-line SAW devices are the most common but there are several different geometries and types of SAW devices. SAW devices can also contain only one IDT. In this configuration they act like resonators and can have very high quality factors. ID desing is covered in detail in section 4.1.1.

\subsection{Review of Group III-Nitride Materials}

Figure 2.1 shows the bandgap $\left(\mathrm{E}_{g}\right)$ energy of the III-N's as a function of the a-lattice parameter. The dashed line represents $\mathrm{E}_{g}$ energies theoretically possible with the ternary alloys and the area enclosed represents the quaternary alloys. There is still debate over the $\mathrm{E}_{g}$ of InN [16], (ranging from $0.7-1.2 \mathrm{eV}$ ), mostly due to the difficulty in growing it as a high quality single crystal. In addition, a miscibility gap in the high In concentration InGaN films results in phase separation during growth and these alloys have proven extremely difficult to grow [17]. And with the availability of high brightness, highefficient GaAs red LEDs, there is currently not much motivation for developing high In concentration InGaN materials. Nerveless, the III-N's remain as the only currently available material system with the ability to cover the entire visible spectrum making them unique. With general lighting applications being responsible for over $20 \%$ of the energy consumption in the United States [18], advances in solid-state lighting have the potential for considerable energy and cost savings. The United States Department of 
Energy predicts that the increased use of solid state lighting will result in a $46 \%$ lighting consumption energy savings by the year 2030 [18]. Smart lighting systems also have the potential for reducing energy costs while providing a means for short distance data transmission via free space optics [19].

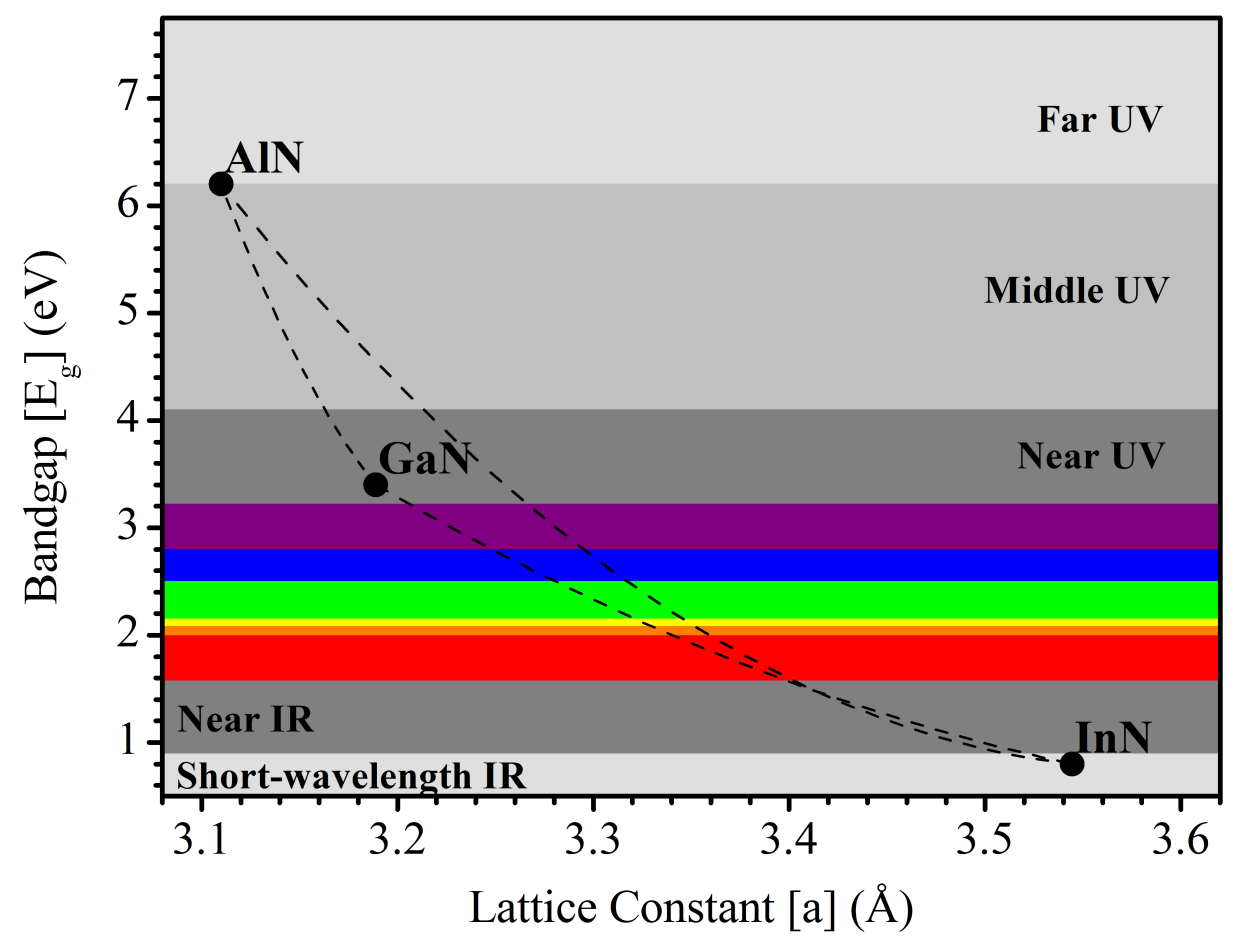

Figure 2.1: Group III-nitride bandgaps and lattice constants. The dashed lines represent the ternary alloys and the enclosed area represents the quaternary alloys.

The III-N's energetically favor, and are most stable in wurtzite crystal structure [20]. Wurtzite is a hexagonal closed packed crystal structure with a bilayer stacking order of ABAB. The III-N's can also form in the cubic or zincblende structure, but this is less common with fewer applications. Wurtzite is asymmetrical in the c-lattice crystal

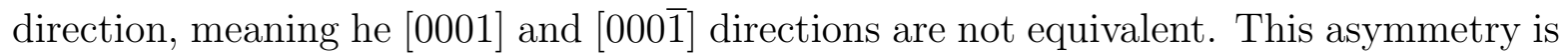
responsible for the piezoelectric properties of the III-N's which have impacts on several different device properties. Piezoelectric fields can have detrimental effects on radia- 
Chapter 2. Background

tive recombination in emitter devices [20], but are also responsible for the high electron sheet density in transistor devices [21]. The piezoelectric properties also make III-N's attractive for electro-acoustic applications like SAW devices detailed in the previous section, and have additionally been studied for micro electro-mechanical systems (MEMS) devices [22]. 


\section{Chapter 3}

\section{Materials and Devices}

\subsection{Group III-Nitride Materials Growth}

All group III-N thin films used in this work were grown in an Aixtron 200/4 RF-S metalorganic chemical vapor deposition (MOVPE) system, which can be seen in Figure 3.1. For these growths, precursors included trimethylgallium (TMGa), trimethylaluminum (TMAl), triethylgallium (TEGa), trimethylindium (TMIn), Bis(cyclopentadienyl)magnesium $\left(\mathrm{Cp}_{2} \mathrm{Mg}\right)$, silane $\left(\mathrm{SiH}_{4}\right)$ and ammonia $\left(\mathrm{NH}_{3}\right)$.

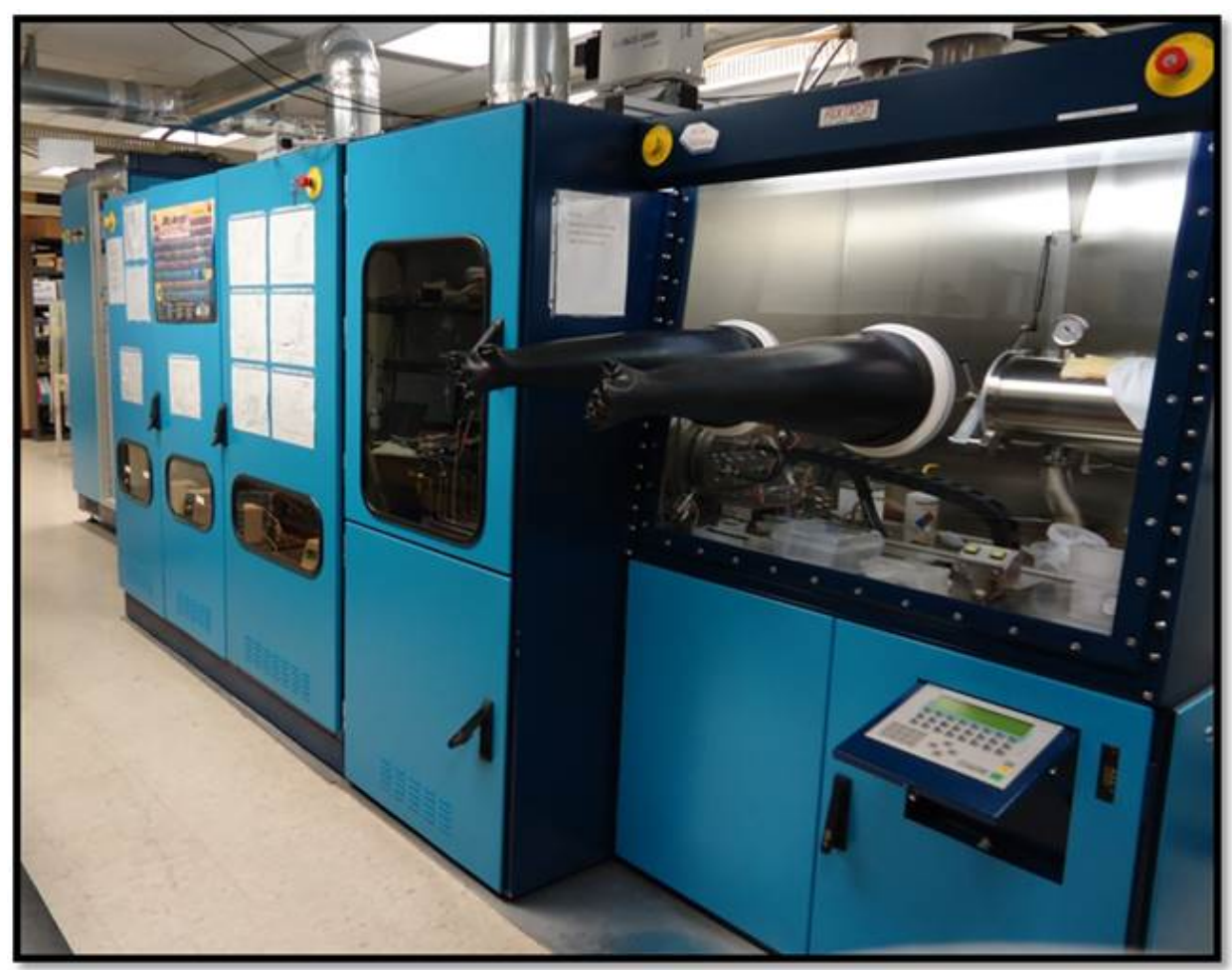

Figure 3.1: Picture of the Aixtron 200/RF-S MOVPE system used for the growth of all III-nitride materils used in this work. 


\subsubsection{Metal Organic Vapor Phase Epitaxy}

Metalorganic vapor phase epitaxy or MOVPE, is a growth technique that involves pyrolysis of the precursors at high temperature and then a chemical reaction at the substrate surface. MOVPE is a very complex growth technique that may require a few hundred parameters to be controlled. In addition, hazardous and harmful precursors require extreme care, especially when replacing the metalorganic precursors. Despite these shortcomings, MOVPE is the clear favorite for the large scale production of GaN-based materials and devices when compared to other growth techniques. Previously, molecular beam epitaxy (MBE) systems provided the highest crystal quality III-N materials, but recent advances in metalorganic precursor technology and improved process control, have closed the gap between MOVPE and MBE III-N crystal quality. In addition, MOVPE does not require ultra-high vacuum, and although complex, MOVPE systems are considerably cheaper to operate and maintain with less downtime then typical MBE systems. Liquid phase epitaxy yields the highest crystal quality films, but a lack of control renders it inadequate for devices where monolayer control of the growth is required.

The MOVPE process starts with the metalorganic (MO) precursors. Early on in the development of MOVPE, a lack as high purity MOs was the limiting factor in the quality of III-N's that could be achieved. But with the commercial success of MOVPE, came the availability of much higher purity precursors. The MOs must be kept at very stable temperatures to ensure a constant vapor pressure during growth. To accomplish this, MOs are kept in baths which regulate the temperature. TMGa, TEGa, and TMAl are kept in the liquid phase while TMIn and $\mathrm{Cp}_{2} \mathrm{Mg}$ are solids at standard bath temperatures. To transport the MO molecules to the growth site, carrier gases are used. Typical carrier gases are nitrogen or hydrogen, and are bubbled up through the MOs, lending to the name of bubbler when referring to MO cylinders. Solid TMIn is the most difficult precursor to control, but advances in bubbler technology have drastically 
increased the reliability of TMIn. Very precise control of the flow and pressure of the MO delivery system are required as well as laminar flow to the growth chamber. Otherwise, precise control of the growth cannot be maintained. This results in a complex system of mass flow and pressure controllers, all of which are computer controlled.

Silane and ammonia are the two gaseous precursors. Very high purity ammonia is required and silane is typically diluted in hydrogen. Large quantities of ammonia are needed due to the high decomposition temperature of ammonia. Only a small percent of ammonia breaks down during the growth process and the flow of ammonia is much greater then the flow of the MOs. After the surface reaction, excess precursors, carrier gases and methane are exhausted from the chamber. This toxic and hazardous gas must be conditioned before it is allowed to be released into the atmosphere. This can be done with filters, chemical scrubbing in acid and combustion to break down any remaining gases.

\subsubsection{GaN for SAW Devices}

All GaN thin films used for SAW devices were grown were grown on AlN buffer layers on sapphire substrates. TMGa, TMAl and $\mathrm{NH}_{3}$ were used as the $\mathrm{Ga}, \mathrm{Al}$ and $\mathrm{N}$ precursors respectively. GaN thin films were grown using $100 \mu \mathrm{mol} / \mathrm{min}$ of TMGa, a V/III ratio of approximately 700 and a reactor temperature of $980{ }^{\circ} \mathrm{C}$.

\subsection{3 $\mathrm{Al}_{x} \mathrm{Ga}_{1-x} \mathrm{~N} / \mathrm{GaN}$ Heterojunctions for HEMTs}

Growth of the ternary alloys (InGaN, AlGaN or InAlN) are considerable more difficult then the growth of the binary alloys. This section focuses on the growth of $\mathrm{Al}_{x} \mathrm{Ga}_{1-x} \mathrm{~N}$ thin films. The incorporation of the $\mathrm{Ga}$ or $\mathrm{Al}$ atoms is largely dependent on temperature, and the surface condition plays a large role in the ternary growth. Temperature gradients can result in films of different stoichiometries across the substrate. In addition, as the ternary alloy grows on the substrate, lattice mismatch creates an increasing strain with 
film thickness. As the strain condition at the surface changes, the relative incorporation of $\mathrm{Ga}$ and $\mathrm{Al}$ changes. This results in films with different stoichiometries as a function of thickness. For the HEMTs in this work, $\mathrm{Al}_{x} \mathrm{Ga}_{1-x} \mathrm{~N}$ films were targeted where $x=0.25$. $\mathrm{Al}_{x} \mathrm{Ga}_{1-x} \mathrm{~N}$ thickness were targeted at $25 \mathrm{~nm}$. The basic HEMT structure can be seen in Figure 3.2.

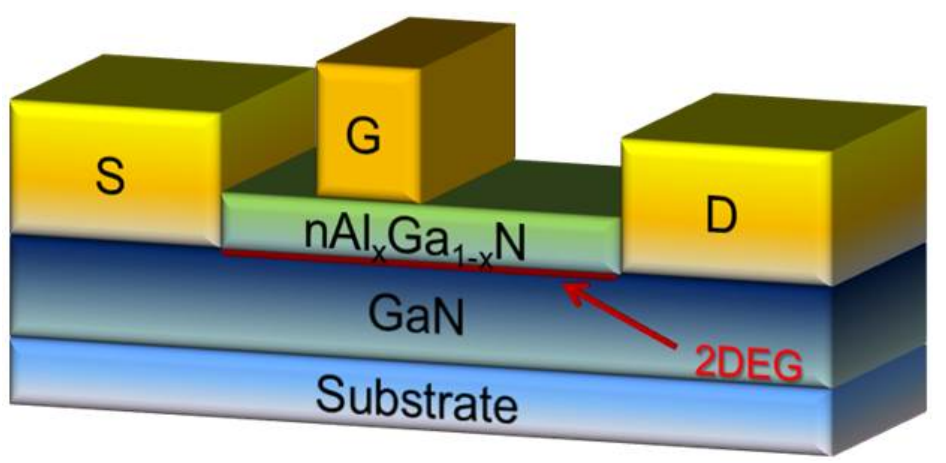

Figure 3.2: Schematic of HEMT epitaxial structure with location of the 2D electron gas shown as a red line.

To achieve the $\mathrm{Al}_{x} \mathrm{Ga}_{1-x} \mathrm{~N}$ conditions required, the $\mathrm{Al}_{x} \mathrm{Ga}_{1-x} \mathrm{~N}$ growth had to be characterized as a function of $x$. For this work the sum of TMGa and TMAl flows were kept at a constant $60 \mu \mathrm{mol} / \mathrm{min}$. First, $x$ was characterized as a function of the ratio of TMAl flowed during growth. Several samples of relatively thick $\mathrm{Al}_{x} \mathrm{Ga}_{1-x} \mathrm{~N}$ thin films were grown on GaN on sapphire. The aluminum concentration was determined using XRD. Figure 3.3 shows the relationship between the aluminum concentration of the $\mathrm{Al}_{x} \mathrm{Ga}_{1-x} \mathrm{~N}$ thin film and the ratio of TMAl. It was found, for an $\mathrm{Al}_{x} \mathrm{Ga}_{1-x} \mathrm{~N}$ thin film, where $x=0.25$, a ratio of $56.67 \%$ was optimal.

Next, the accurate growth rate of the $\mathrm{Al}_{x} \mathrm{Ga}_{1-x} \mathrm{~N}$ thin films needed to be determined for the growth of precise thicknesses. Again, several thick AlGaN films were grown. The growth rate was monitored using a LayTec EpiRas system. The resulting growth rate of the $\mathrm{Al}_{x} \mathrm{Ga}_{1-x} \mathrm{~N}$ thin film was found to be dependent on III/V ratio as well as TMAl ratio and growth chamber pressure, and can be seen in Figure 3.4.

HEMT devices in this work consisted of two layers, a $25 \mathrm{~nm}$ of Si-doped $\mathrm{nAl}_{x} \mathrm{Ga}_{1-x} \mathrm{~N}$ 


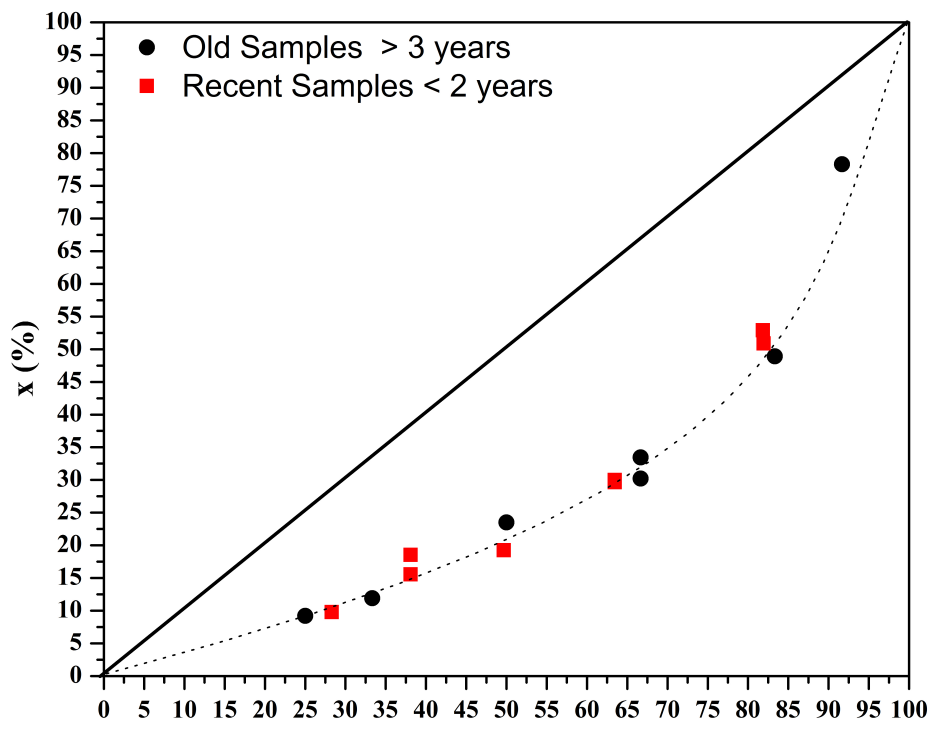

TMAI/(TMAI+TMGa)

Figure 3.3: AlN concentration in $A l_{x} G a_{1-x} N$ thin films as a function of $\frac{T M A l}{T M A l+T M G a}$.

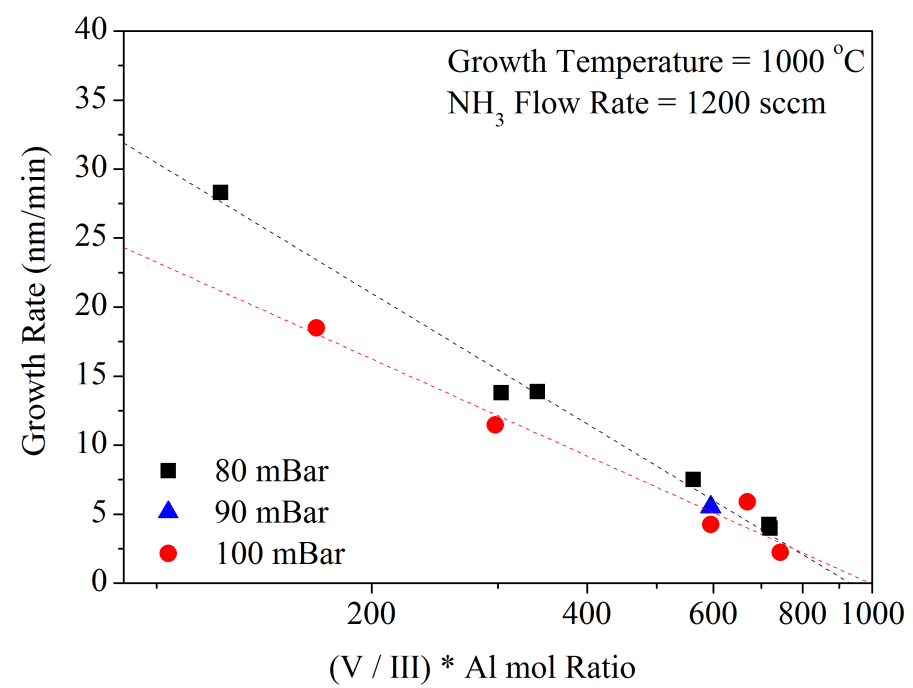

Figure 3.4: Growth rate of $A l_{x} G a_{1-x} N$ as a function of III/V ratio multiplied by $\frac{T M A l}{T M A l+T M G a}$. 
on a thick epilayer of insulating $\mathrm{GaN} . \mathrm{Al}_{x} \mathrm{Ga}_{1-x} \mathrm{~N}$ films were grown with TMAl at $34 \mu \mathrm{mol} / \mathrm{min}$, TMGa at $26 \mu \mathrm{mol} / \mathrm{min}$ and a V/III ratio of approximately 890 . For $\mathrm{Al}_{x} \mathrm{Ga}_{1-x} \mathrm{~N}$ films, the reactor temperature was $1000{ }^{\circ} \mathrm{C}$ and the chamber pressure was kept at $80 \mathrm{mBar}$.

\subsection{Device Fabrication}

All devices for this work were fabricated in the West Virginia University (WVU) Shared Research Facilities Cleanroom. Fabrication of devices consisted of standard semiconductor photolithography, etch, and metalization processes. The recipes detailed in the following sections were accurate at the time of fabrication. However, process parameters will drift over time and will vary from toolset to toolset. Therefore, verification and optimization of the processes developed for this work may be needed to achieve the same results, even if performed using the exact same equipment. A list of the equipment used and their purpose is below. The website for the WVU Shared facilities is given at the end of the list.

- Temescal BJD-2000 Electron Beam Evaporation System for metallic contact evaporation

- Oxford Plasmalab 80+ PECVD System for $\mathrm{SiO}_{x}$ and $\mathrm{SiN}_{x}$ deposition

- CVC 610 DC Magnetron Sputtering System for gold bonding pad deposition

- Suss Microtech MA6 Mask Aligner for photolithography

- Trion ICP Reactive Ion Etching System for device mesa etching

- AnnealSys AS-Micro Rapid Thermal Annealer for device annealing

- West Bond 74776E Gold Wire Bonder for connecting electrical leads between devices and packages 
- <http://sharedresearchfacilities.wvu.edu/>

\subsubsection{SAW Device Fabrication}

SAW devices were fabricated using traditional image reversal, optical photolithography and liftoff techniques. For room temperature devices, titanium and aluminum bilayers were deposited onto the patterned samples using e-beam evaporation. The titanium layers were necessary to provide adequate adhesion between the aluminum IDTs and the GaN thin films. To minimize mass loading effects, the Ti layers were kept as thin as possible, and IDTs consisted of 2/100 nm of Ti/Al. The samples were then placed in a bath of acetone to perform liftoff. Samples were cleaned with methanol and air-dried with nitrogen. Finally, samples were annealed 5 min at $300{ }^{\circ} \mathrm{C}$ in a nitrogen environment.

For devices used at high temperatures, the $\mathrm{Ti} / \mathrm{Al}$ bilayers were replaced with nickel and platinum. The nickel served as the adhesion layer between platinum and GaN. Bi-layers were deposited with a thickness goal of 2 and $80 \mathrm{~nm}$ for $\mathrm{Ni}$ and $\mathrm{Pt}$, respectively. Liftoff was the same for high temperature devices, but the annealing was done for $5 \mathrm{~min}$ at $300{ }^{\circ} \mathrm{C}$ in an atmospheric environment. A detailed procedure for SAW device fabrication can be found in Appendix B.

\subsubsection{HEMT Fabrication}

In addition to a more complex epitaxial structure, HEMT device fabrication was much more complicated then the SAW devices. Several iterations of the process were performed and optimized until a suitable recipe was achieved which was reliable and repeatable. The first step that was optimized was the mesa etch step. Etch rates of $\mathrm{Al}_{X} \mathrm{Ga}_{1-x} \mathrm{~N}$ vary as a function of $x$ for the same recipe and a very precise etch depth was required for repeatable device operation. Several samples of AlGaN with different $\mathrm{Al}$ concentrations were grown. Samples were cut into several pieces. A set of samples was patterned and etched in the same batch to eliminate any human error or tool drift 
from the measurement. The following chamber conditions were used for all etch tests:

\begin{tabular}{ll}
\hline Process & Setting \\
Parameter & \\
\hline ICP Power & $300 \mathrm{~W}$ \\
RIE Power & $100 \mathrm{~W}$ \\
Pressure & $10 \mathrm{mTorr}$ \\
$\mathrm{BCl}_{3}$ Flow & $10 \mathrm{sccm}$ \\
$\mathrm{Cl}_{2}$ Flow & $20 \mathrm{sccm}$ \\
Time & $300 \mathrm{sec}$
\end{tabular}

Multiple batches were processed on different days to ensure repeatability. Etch depth was measured with stylus profilometer. Figure 3.5 shows the etch rate of $\mathrm{Al}_{X} \mathrm{Ga}_{1-x} \mathrm{~N}$ as a function of $\mathrm{Al}$ concentration. The dashed line is a linear fit to the data.

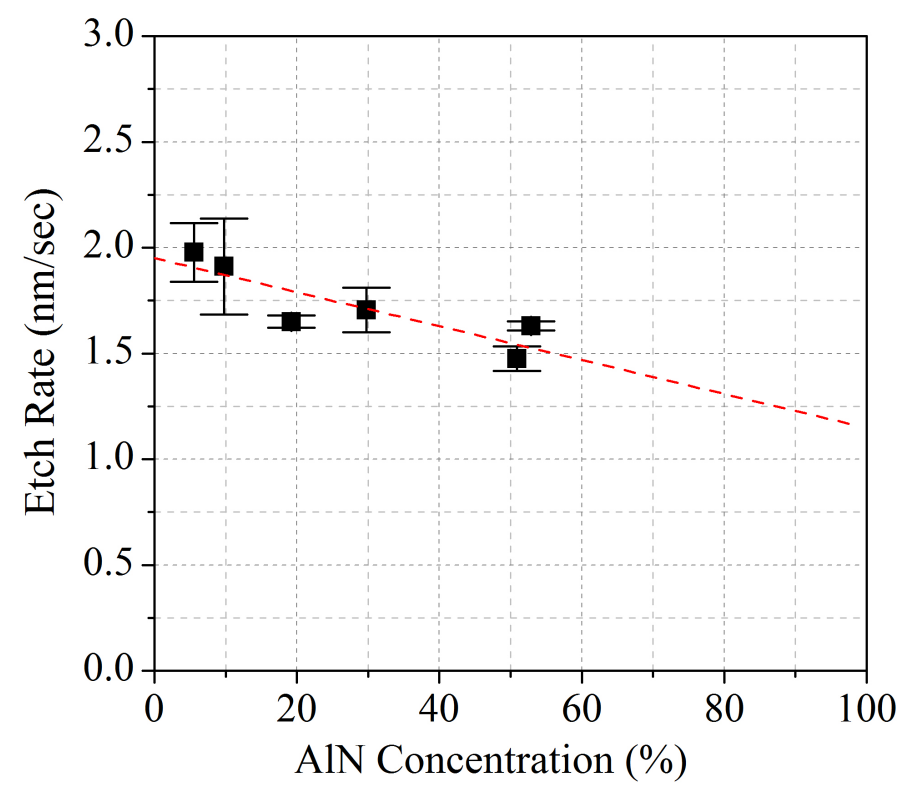

Figure 3.5: ICP etch rate of $A l_{x} G a_{1-x} N$ as a function of $x$.

The electrostatic chuck on the ICP has been changed since this etch test was performed. This result is no longer valid, however the method prescribed for achieving a relationship between $\mathrm{Al}$ concentration of $\mathrm{AlGaN}$ and etch rate can be used for future 
etch optimizations.

Next, the Ohmic contacts were developed and optimized using the transmission line method (TLM). TLM gives the specific contact resistance as a function of area of the contact pad. TLM is performed by depositing several rectangular pads onto the semiconductor surface. IV measurements are made between several adjacent pads with different spacings. The total resistance is plotted as a function of contact spacing, as shown in Figure 3.6. The total resistance measured is the sum of all resistances between the measurement probes. This includes the resistance in the semiconductor itself and the resistance between the probe tips and the contact pad. For this measurement, the following assumptions are made:

- All resistances remain constant for each measurement.

- The resistance between the probe tips and the contact pad are negligible.

- The resistance in the doped semiconductor is small relative to the contact resistance when the contacts are very close, and can be neglected.

- No current travels outside the smallest rectangle that include both contacts being measured. This is not true, but can be enforced if the contacts are deposited onto etched Mesas.

A linear fit to the data results in a non-zero y-intercept. Although not experimentally possible to measure, intuitively, this value is the resistance that would be measured if there was no space between the contact pads. Given the above assumptions, the $y$-intercept is then twice the contact resistance, $2 R_{C}$, since two contacts are used in each measurement. The $\mathrm{x}$-intercept, $2 \mathrm{~L}_{\mathrm{T}}$, is the transfer length, or the width of the contact for which current is flowing from the contact into the semiconductor. Although not used to calculate specific contact resistance, this value cannot exceed the width of the contacts. This is a good check to ensure the results of the measurement are reasonable. 


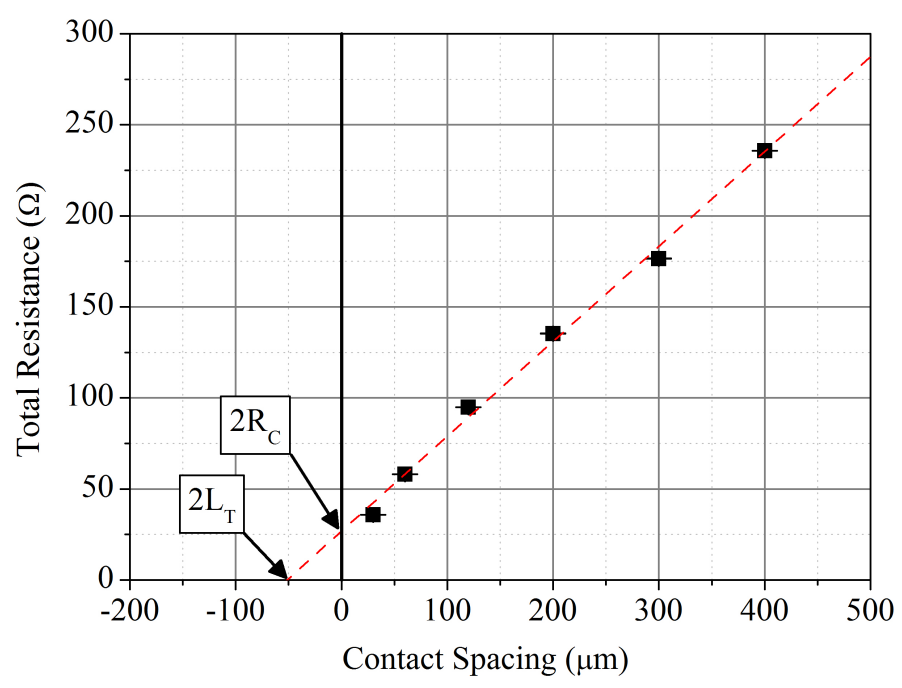

Figure 3.6: Total Resistance measured as a function of contact spacing. The specific contact resistance is one half of the $y$-intercept of the linear curve fit (shown as a red dashed line).

Once the contact resistance, $R_{C}$, is known, the specific contact resistance is proportional to the area of the contact. A more detailed explanation of the calculation to determine specific contact resistance from TLM can be found in $[23,24]$. The specific contact resistance and the transfer length of three different metal stacks on the AlGaN/GaN heterostructure can be found in Figure 3.7. Although many assumptions were made, and the TLM measurement is prone to several sources of error, the exact value of the specific contact resistance is not needed. Only the relative contact resistances between the recipes is of interest at this stage in development. Of the Ohmic contact processes tested, it was found that a metal stack consisting of 30/225/80/40 nm of $\mathrm{Ti} / \mathrm{Al} / \mathrm{Ni} / \mathrm{Au}$, which was annealed in nitrogen for 30 seconds at $850{ }^{\circ} \mathrm{C}$ had the lowest contact resistance.

After the AlGaN etch was characterized, and the Ohmic contacts were optimized, several iterations of HEMT device fabrication were performed. A detailed procedure for the best process obtained during this work can be found in Appendix C. Figure 3.8 shows an optical image of a fabricated HEMT device using the process outlined in C. 


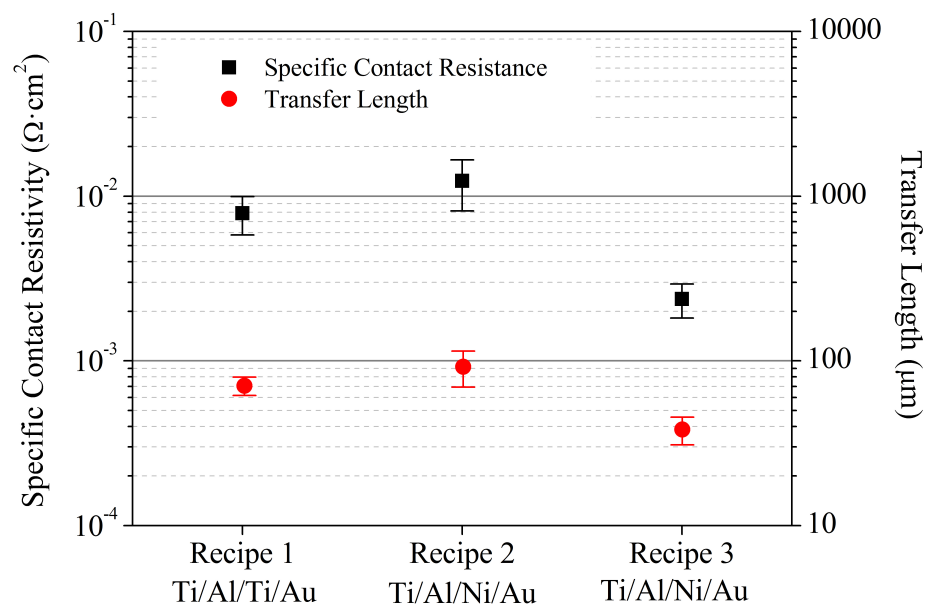

Figure 3.7: Specific contact resistivity and transfer length of three different metal stacks on $\mathrm{AlGaN} / \mathrm{GaN}$.

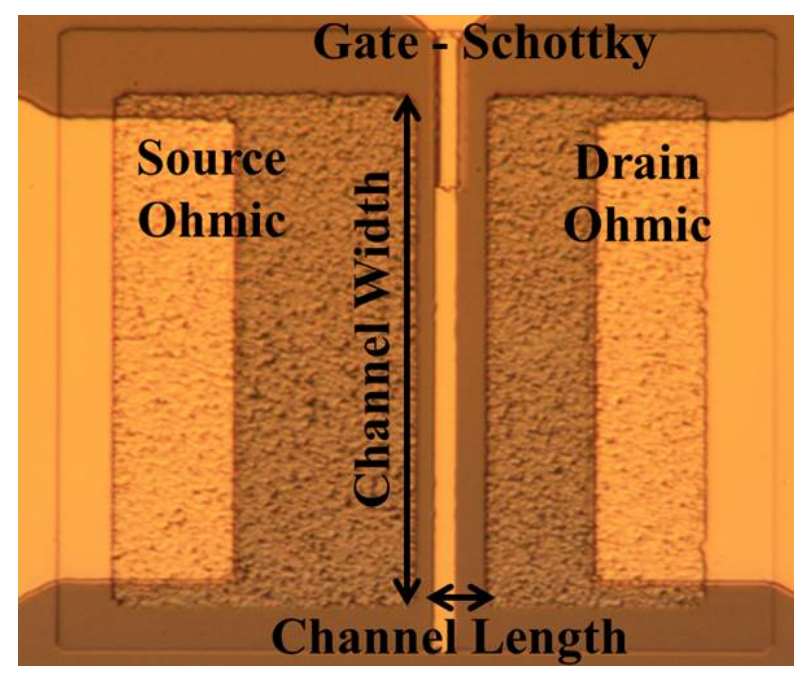

Figure 3.8: Optical image of a fabricated HEMT device with important parameters labeled.

The effect of annealing the Ohmic contact is clearly visible as a roughening of the contact surface, when compared to the Schottky contact and bonding pad. Aluminum has a melting point of approximately $660{ }^{\circ} \mathrm{C}$, and this roughening of the contact is a result of the relatively thick aluminum layer melting and balling up during the annealing process. 


\subsection{Electro-Acoustic Characterization}

Electro-acoustic measurements were performed using an Agilent E8362B Vector Network Analyzer in side a Faraday cage to eliminate any outside microwave interference. Samples were probed using Cascade Microtech ACP40-GSG-150 probes and were connected to the VNA with Cascade Microtech 101-162-B $40 \mathrm{GHz}$ cables. To minimize any losses in the test setup, calibrations to the end of the probe tips were performed using the Cascade Microtech 101-190 impedance standard substrate (ISS). calibration and operation procedures can be found in Appendix D.

There are two main types of network analyzers: scalar network analyzers (SNAs) and vector network analyzers (VNAs). SNAs only measure the amplitude of a signal and its reflections, while VNAs measure both the amplitude and phase of a signal. Common network analyzer terminology has the incident wave measured with the $\mathrm{R}$ (for reference) receiver. The reflected wave is measured with the A receiver and the transmitted wave is measured with the $\mathrm{B}$ receiver. With amplitude and phase information of these three waves, we can quantify the reflection and transmission characteristics of our device under test (DUT). Some of the common measured terms are scalar in nature (the phase part is ignored or not measured); while others are vector (both magnitude and phase are measured). For example, return loss is a scalar measurement of reflection, while impedance results from a vector reflection measurement. Some, like group delay, are purely phase-related measurements [25].

There are three main sources for measurement error: systematic, random, and drift. Systematic errors are due to imperfections in the analyzer and test setup. They are repeatable (and therefore predictable), and are assumed to be time invariant. Systematic errors are characterized during the calibration process and mathematically removed during measurements. Random errors are unpredictable since they vary with time in a random fashion. Therefore, they cannot be removed by calibration. The main con- 
tributors to random error are instrument noise (source phase noise, sampler noise, IF noise). Drift errors are due to the instrument or test-system performance changing after a calibration has been done. Drift is primarily caused by temperature variation and it can be removed by further calibration(s). The timeframe over which a calibration remains accurate is dependent on the rate of drift that the test system undergoes in the users test environment. Providing a stable ambient temperature usually goes a long way towards minimizing drift [25]. 



\section{Chapter 4}

\section{Surface Acoustic Wave Devices}

\subsection{Introduction}

\subsubsection{Interdigital Transducer Design}

Surface acoustic waves can be generated in a piezoelectric material through the use of comb-like interdigital transducers (IDTs). Figure 4.1 shows the basic geometry of a normal IDT. The individual teeth of the comb-like IDT are known as fingers. The finger width, $a$, is shown in Figure 4.1. The finger spacing is then equal to $p-a$. The metallization ratio of an IDT, $\eta$, is equal to $a / p$. For a metallization ratio of $50 \%$, $a=p / 2$, and the finger width and finger spacing are equal. This is the standard $\eta$ for normal IDTs, but other $\eta$ 's can be used depending on the desired operation*. The period of an IDT, $T$ is equal to $2 p$. When an electric potential is applied to the IDT, the piezoelectric material undergoes a mechanical deformation. In the case of a sinusoidal alternating potential, the mechanical deformation is also sinusoidal. As the material deforms in the $x$ direction, it will also deform in the $y$ and $z$ directions proportional to Poisson's ratio, $\nu_{P}^{\dagger}$. This is the origin of the surface acoustic wave, which is launched from both ends of the IDT. This is know as a bi-directional IDT, but is simply referred to as an IDT, or normal IDT in this work.

Like all waves, the velocity, frequency and wavelength of a SAW are related by Equation (4.1), where $f$ is the frequency of the SAW, $\nu$ is the acoustic velocity of the

\footnotetext{
*An example when $\eta \neq 0.5$ is described in Section 4.3 .1

†Here the subscript $\mathrm{P}$ is used to differentiate between Poisson's ratio and acoustic velocity.
} 
material and $\lambda$ is the wavelength of the SAW.

$$
\nu=f \lambda
$$

When talking about SAW devices, it is important to define what is meant by frequency and wavelength. When reading about SAW devices in the literature, one will come across several terms for frequency that may be used interchangeably, including, but not limited to, center frequency, characteristic frequency, natural frequency, fundamental frequency, resonating frequency and operating frequency. In this work, the characteristic frequency, $f_{0}$, and operating frequency, $f_{O p}$, will be used to discuss SAW device operation and are defined in Equation (4.2) and Equation (4.3), respectively. The characteristic frequency is a calculated frequency based on material properties and device design. Operating frequency is an experimentally measured value for a given SAW device. The term fundamental frequency is also used and refers to lowest frequency at which a specific

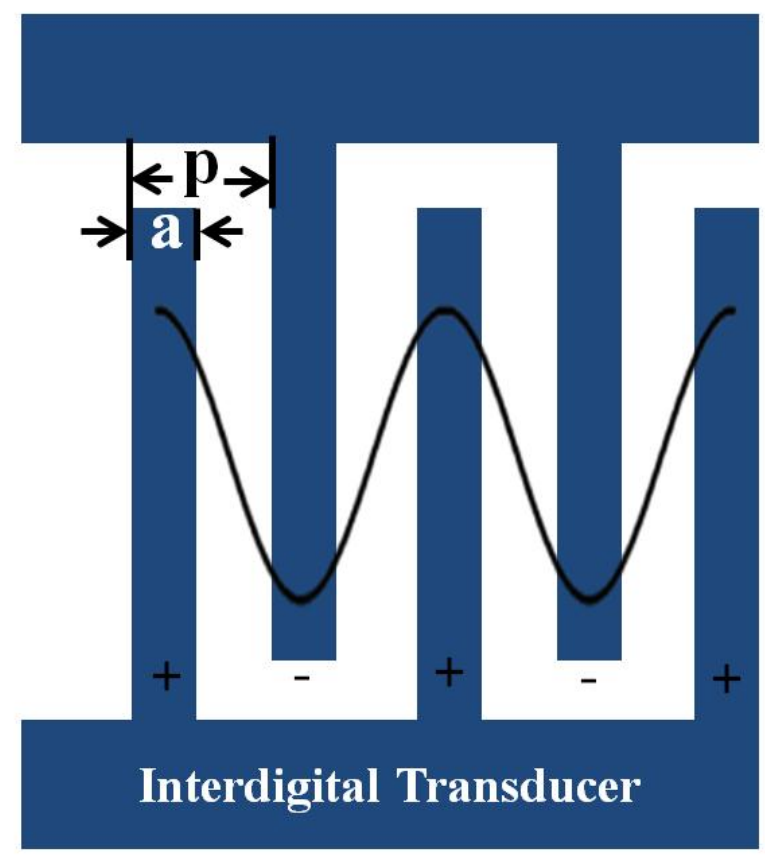

Figure 4.1: Basic design of an interdigital transducer used to generate surface acoustic waves in piezoelectric materials [26]. Metallization ratio, $\eta=a / p$. 
mode can occur.

For $f_{0}, \nu_{0}$ is the theoretical acoustic velocity of the bulk piezoelectric material, and $\lambda_{0}$ is the characteristic wavelength of the SAW device determined by IDT design, where $\lambda_{0} \equiv T$. When $f_{O p}$ is used, $\nu$ is the measured acoustic velocity* of a SAW, and $\lambda_{e f f}$ is the effective wavelength of the SAW device. For a normal IDT designed to operate at the fundamental frequency, $\lambda_{e f f}=\lambda_{0}$.

$$
\begin{gathered}
f_{0}=\frac{\nu_{0}}{\lambda_{0}} \\
f_{O p}=\frac{\nu}{\lambda_{e f f}}
\end{gathered}
$$

As a first approximation when designing a basic IDT, one can assume the acoustic velocity is constant. Once a SAW device is fabricated, the period of the IDT, $T$, is fixed and $\lambda_{0}$, is constant. Therefore, $f_{0}$ of the SAW device depends on the material used and the size of the IDT fingers. If the acoustic velocity of a material is known, designing an IDT to operate at a specific frequency is trivial. As the size of the IDT decreases, however, the fabrication of the device may become quite complex and expensive. SAW IDT fabrication is covered in detail in Section 3.2.1. There are several second order effects which can complicate the design of a SAW device. There is generally a trade off between size, simplicity and performance. A list of several second order effects associated with SAW devices can be found in Appendix A, along with a few design solutions to help minimize these effects.

${ }^{*}$ Piezoelectric film thickness, mass loading from the fabricated IDTs, choice of substrate and cracking are a few of the parameters that can cause the actual acoustic velocity of a SAW to differ from the theoretical bulk acoustic velocity and are covered in Section 4.4. 


\subsubsection{SAW Devices as Chemical Sensors}

Since the energy of a SAW is confined to the surface of the material, the frequency response of a SAW device will change in response to any change in the surface density [27]. This makes SAW devices very sensitive to mass loading. One method for fabricating a SAW sensor is to add an active layer on the piezoelectric surface between the input and output IDTs of a SAW device. This material is chosen for its selectivity and ability to absorb or bind to the species, trapping it on the surface [28]. As the surface becomes loaded, the resonant frequency of the SAW device will shift to lower frequencies and the insertion loss will increase due to surface dampening as illustrated in Figure 4.2. The sensitivity of a SAW device to mass loading is shown in Equation (4.4), where $S$ is the sensitivity factor, $c_{m}$ is a material constant and $f_{0}$ is the operating frequency of the SAW device [5] .

$$
S=-c_{m} f_{0}^{2}
$$

Given this relationship, it becomes obvious that the higher the operating frequency of a SAW device, the more sensitive the device is when operating as a sensor. It is theorized that SAW devices could have sensitivities on the order of parts per trillion and even be

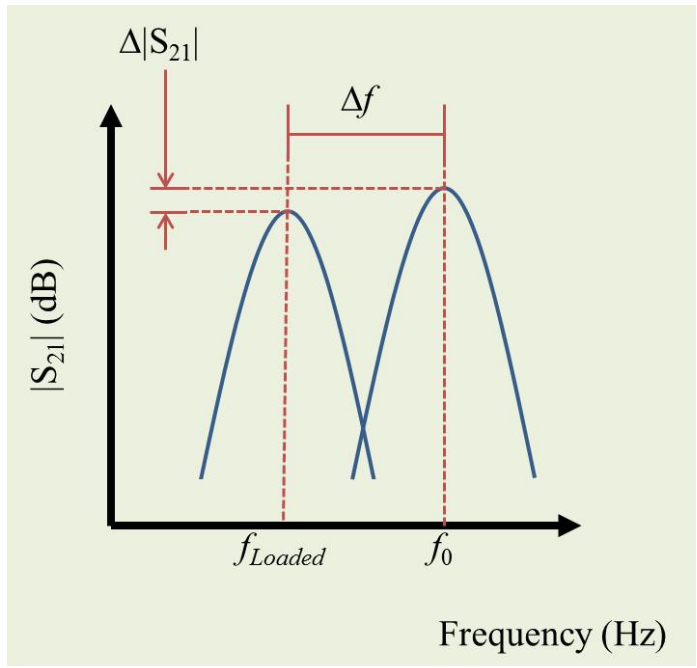

Figure 4.2: Mass loading effects on the frequency response of a SAW device, allowing them to be used as chemical sensors [26]. 
able to sense a single molecule resting on its surface [29].

\subsection{GaN Based Surface Acoustic Wave Devices}

The most common materials for SAW devices include lithium niobate $\left(\mathrm{LiNbO}_{3}\right)$, lithium tantalate $\left(\mathrm{LiTaO}_{3}\right)$, quartz, lead zirconium titanate $\left(\mathrm{Pb}\left[\mathrm{Zr}_{x} \mathrm{Ti}_{1-x}\right] \mathrm{O}_{3}\right.$ or PZT), zinc oxide $(\mathrm{ZnO})$ and langasite $\left(\mathrm{La}_{3} \mathrm{Ga}_{5} \mathrm{SiO}_{14}\right.$ or LGS). Recently, there has been much work done using the group III-nitride family of materials for the active piezoelectric layer in SAW devices [30-41]. The majority of this work focuses on SAW devices based on AlN or AlGaN/GaN modulation doped heterostructures. There is little to be found in the literature regarding GaN based SAW devices. The work and experimental results detailed in this chapter focus on SAW devices on GaN thin films and a few of their applications.

When discussing SAW devices on thin films, it is important to understand the $k h$ parameter, referred to in this work as $k h_{\mathrm{GaN}}$. Here, $k=2 \pi / \lambda_{0}$, is the wavenumber and the $k h_{\mathrm{GaN}}$ parameter relates the characteristic wavelength, $\lambda_{0}$, to the GaN thin film thickness, $h$. If $\lambda_{0}=h$, then $k h_{\mathrm{GaN}}=2 \pi$. It is a valid first approximation to assume all the energy of a SAW is contained within one wavelength of the surface. Therefore, for

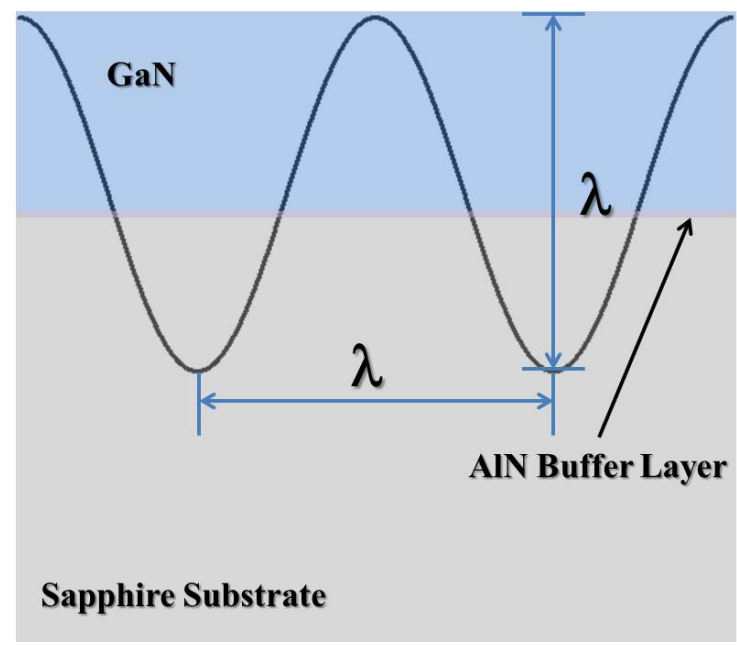

Figure 4.3: Schematic showing the cross sectional view of a SAW traveling in a GaN thin film on a sapphire substrate. 
$k h_{\mathrm{GaN}}>2 \pi$, the SAW only travels within the GaN thin film, and the acoustic velocity, $\nu$, of the SAW is approximately equal to the acoustic velocity of bulk GaN. If $k h_{\mathrm{GaN}}<2 \pi$, then the SAW travels in the GaN thin film and the sapphire substrate, as illustrated in Figure 4.3. In this case, $\nu$ is some convolution of the acoustic velocities of bulk Gan and sapphire. This is an important effect of SAW devices on thin films and is discussed in detail in Section 4.4

\subsubsection{Frequency Response of SAW Devices on GaN}

Gallium nitride thin films for this work were grown on C-plane sapphire substrates via metal organic vapor phase epitaxy, which is detailed in Section 3.1.1. SAW devices discussed in this section were fabricated on several different samples of GaN with film thicknesses of 4.0 and $1.6 \mu \mathrm{m}$. SAW devices were also deposited on AlN on (111) silicon. SAW devices were fabricated with traditional optical photolithographic and

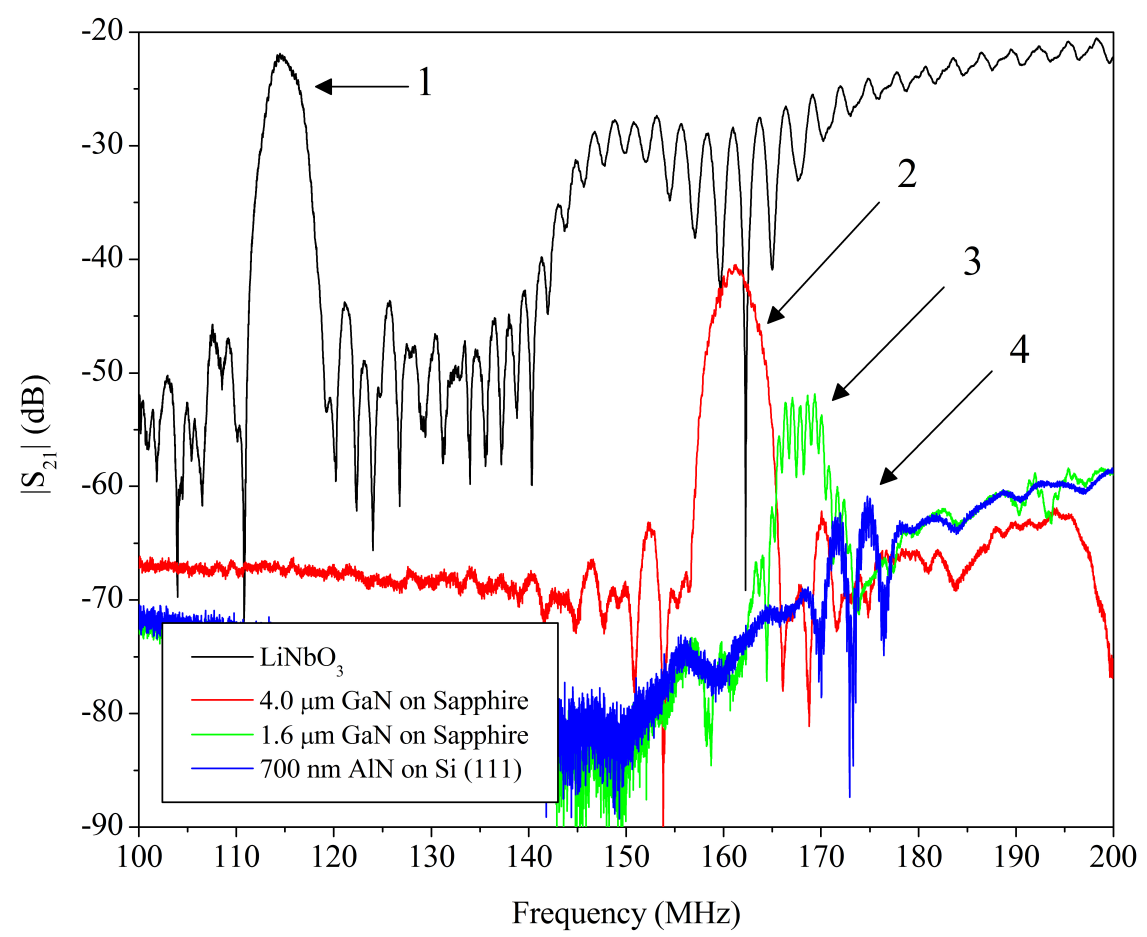

Figure 4.4: Frequency response of SAW devices on 1) lithium niobate, 2) 4.0 $\mu \mathrm{m}$ GaN on sapphire, 3) $1.6 \mu \mathrm{m}$ GaN on sapphire and 4)700 $\mathrm{nm}$ AlN on $\mathrm{Si}(111)$. 
metallization procedures, which are described in Section 3.2.1. In addition, SAW devices were fabricated on $2 \mathrm{inch}, 41^{\circ} \mathrm{Y}$-cut $\mathrm{LiNbO}_{3}$ wafers. The frequency response of all SAW device was measured using a vector network analyzer. The measurement technique is discussed thoroughly in Section 3.3.

Comparison of typical device responses can be seen in Figure 4.4. The responses shown here are for the fundamental Rayleigh mode SAW. The devices measured had finger widths and spacing of $8 \mu \mathrm{m}$, where $\lambda_{0}=32 \mu \mathrm{m}$. While SAW devices on GaN films show higher insertion loss than those on $\mathrm{LiNbO}_{3}$, the $4.0 \mu \mathrm{m}$ film showed comparable sidelobe rejection and overall device response. AlN films were only grown to $700 \mathrm{~nm}$ and the device response on these films was relatively poor. The AlN thin films were grown on conducting silicon substrates and the poor frequency response is most likely attributed to electromagnetic feedthrough $(\mathrm{EF})^{*}$.

\subsubsection{Non-Rayleigh SAW Modes in GaN}

The fundamental Rayleigh mode is the first (or slowest) surface acoustic mode that can propagate within a given material. It is also referred to as the $0^{\text {th }}$ order mode or the 0 mode. The next mode beyond the Rayleigh mode is known as a Sezawa mode wave, referred to as the $1^{\text {st }}$ order mode or the 1 mode. Higher modes are not given names and are simply referred to as the $2^{\text {nd }}$ order mode, $3^{\text {rd }}$ order mode, etc.

Unlike the Rayleigh mode waves, Sezawa mode waves can only propagate along certain crystal axes in GaN, as shown in Figure 4.5. The reference flat on a C-plane sapphire wafer corresponds to the $(11 \overline{2} 0)$ plane. For SAW devices aligned perpendicular and parallel the the flat, the SAW propagates in the [11 $\overline{2} 0]$ and [1 $\overline{1} 00]$ directions respectively ${ }^{\dagger}$. The Rayleigh mode SAW frequency response is present in all three samples regardless of device orientation with the substrate. The Sezawa mode SAW frequency

\footnotetext{
${ }^{*} \mathrm{EF}$ is described in detail in Section 4.7

${ }^{\dagger}$ This is illustrated in Figure 4.10(a).
} 
response is only present in devices oriented perpendicular to the sapphire reference flat. In $\mathrm{GaN}$ on sapphire, the Sezawa mode wave can propagate in the [11 $\overline{2} 0]$ direction but is suppressed in other directions.

The SAW devices in Figure 4.5, were fabricated on $6.0 \mu \mathrm{m}$ thick GaN films. The devices had finger widths and spacings of $2 \mu \mathrm{m}$ with $\lambda_{0}=8 \mu \mathrm{m}$ and $k h_{\mathrm{GaN}}=4.71$. The Rayleigh mode SAW response is at $513 \mathrm{MHz}$, which corresponds to an acoustic velocity of $4104 \mathrm{~m} / \mathrm{s}$; higher then the $3820 \mathrm{~m} / \mathrm{s}$ acoustic velocity for Rayleigh mode SAWs in very thick GaN films [42]. GaN on sapphire is a slow on fast crystal structure. This structure effects the acoustic velocity of the SAW modes and is discussed in more detail in Section 4.4. The acoustic velocity of these modes in the slow on fast system can be theoretically calculated using perturbation theory [43].The Sezawa mode SAW response in the $[11 \overline{2} 0]$ direction is at $1.435 \mathrm{GHz}$, corresponding to an acoustic velocity of $11,480 \mathrm{~m} / \mathrm{s}$, slightly

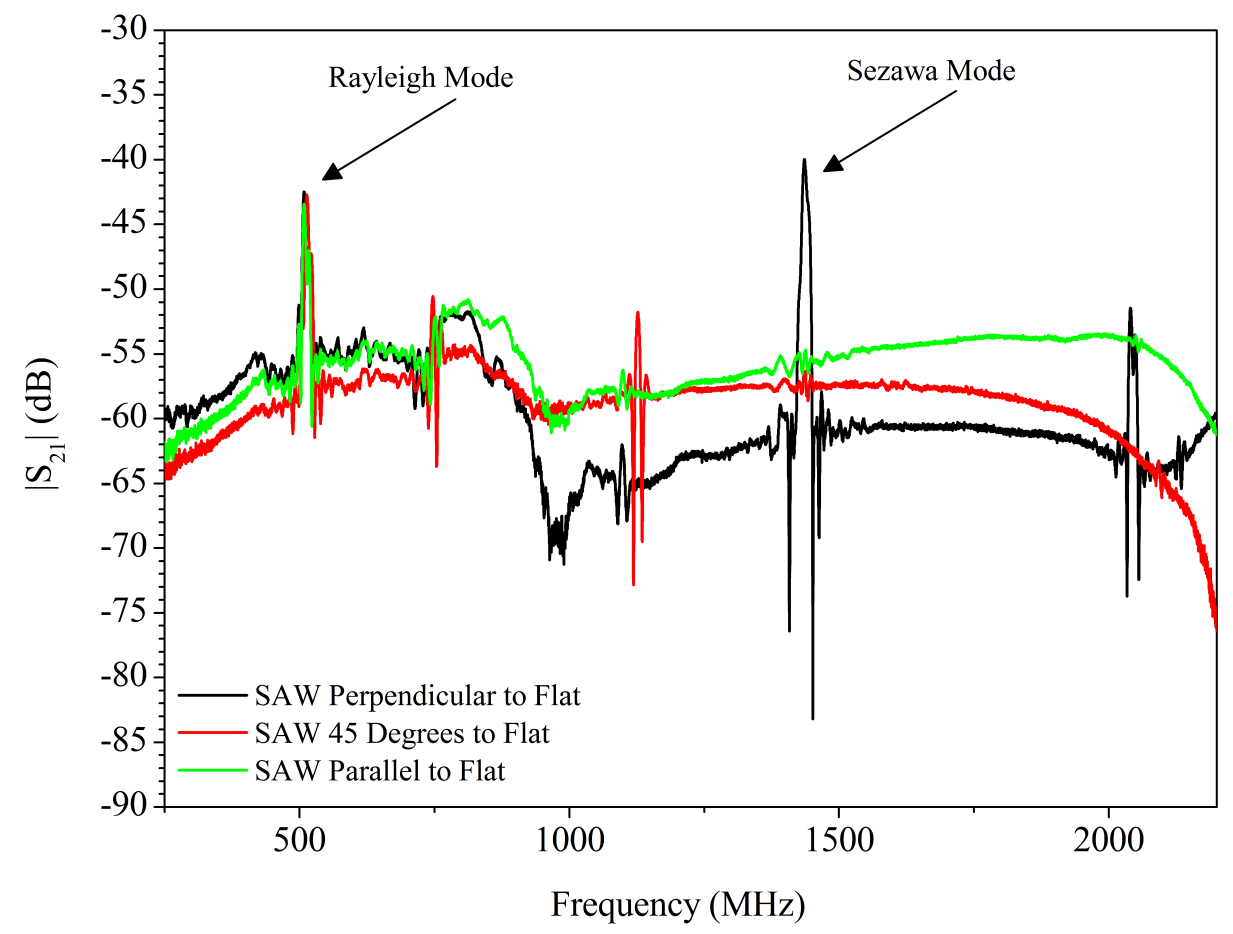

Figure 4.5: Wideband frequency response of SAW devices on GaN thin films showing Rayliegh mode SAWs and Sezawa mode SAWs. Propagation of non-Rayleigh mode SAWs depends on crystal orientation. 
higher than the longitudinal acoustic velocity for sapphire. This mode is known as a pseudo-Sezawa SAW, and will only propagate in the GaN on sapphire systems with a small $k h_{\mathrm{GaN}}$ value* [44]. While there is much literature regarding the use of the Sezawa mode for SAW devices and sensors [41,45-49], it is beyond the scope of this work and is not discussed further.

\subsection{Harmonic SAW Devices}

The acoustic velocity of Rayleigh mode SAWs in thick $\mathrm{GaN}^{\dagger}$ is less then $4000 \mathrm{~m} / \mathrm{s}$. According to Equation (4.2), to obtain a Rayleigh mode SAW device with $f_{0}>1 \mathrm{GHz}$, $\lambda_{0}$ must be less then $4 \mu \mathrm{m}$. For a normal IDT, where the finger width and finger spacing are equal to $\lambda_{0} / 4$, this requires sub-micron fabrication. Using normal IDTs, achieving $\mathrm{GHz}$ range operation without sub-micron fabrication requires a different SAW mode to be chosen, i.e. the Sezawa mode or higher order modes. A harmonic device design is used in this work to make Rayleigh mode SAW devices which operated in the $\mathrm{GHz}$ regime without sub-micron fabrication. When a SAW IDT is excited, higher harmonic SAWs are generated in addition to the fundamental. These harmonic modes can be selected through IDT design.

\subsubsection{Harmonic SAW Device Design}

The basic design of a harmonic SAW device is shown in Figure 4.6, and is a steppedtransducer design similar to that proposed by P. Naraine et al. [50]. This type of harmonic IDT design is referred to as an HIDT in this work. To select a given harmonic, the HIDT is dived into the corresponding number of tracks, $S_{n}$. For the fifth harmonic, $n$ $=5$; for the seventh harmonic, $n=7$, etc. These tracks are offset by a distance $W$ equal to $\lambda_{0} / n$. For the case of a fifth harmonic device with $\lambda_{0}=20 \mu \mathrm{m}$, each track is offset

\footnotetext{
${ }^{*}$ Here, small refers to $k h_{\mathrm{GaN}}<2 \pi$

${ }^{\dagger}$ Here, thick GaN is defined as a GaN thin film whose thickness is greater then the characteristic wavelength, $\lambda_{0}$. In other words, $k h_{\mathrm{GaN}}>2 \pi$. This is explained in more detail in Section 4.4.
} 

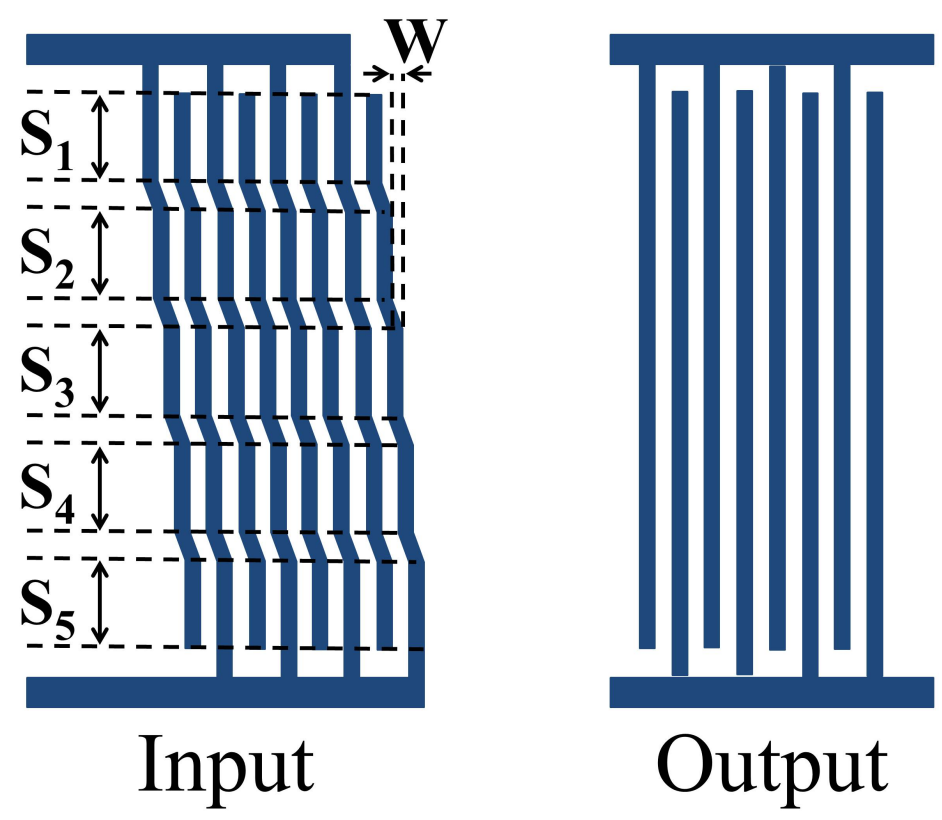

Figure 4.6: Basic geometry of a SAW device designed to operate at the 5th harmonic [26].

by $4 \mu \mathrm{m}$. The surface waves launched by the input HIDT will interfere on the normal output IDT. Due to the phase difference of the waves in each track, the harmonic waves experience constructive interference while the fundamental waves undergo deconstructive interference. This is demonstrated mathematically in Equations (4.5) and (4.6) for the fundamental and fifth harmonic cases respectively.

$$
\begin{gathered}
U_{\text {Fundamental }}=\sum_{n=1}^{5} U_{0} e^{i \frac{n 2 \pi}{5}} \equiv 0 \\
U_{\text {Fifth }}=\sum_{n=1}^{5} U_{0} e^{5 i \frac{n 2 \pi}{5}} \equiv 5 U_{0}
\end{gathered}
$$

The design parameters of the harmonic SAW devices used in this work are shown in Table 4.1. It is important to note that $\eta$ for the seventh and eleventh harmonic cases is not equal to $50 \%$. Harmonic SAW efficiency is related the $\eta$ and was theoretically calculated up to the eleventh harmonic by Campbell and Edmonson in 2002 [51]. To 
avoid sub-micron finger widths or spacing, metallization ratios are chosen as close to 0.5 as possible. For the seventh harmonic, peak efficiencies are at approximately $\eta=0.35$ and 0.65. For the eleventh harmonic, peak efficiencies are at approximately $\eta=0.40$ and 0.60 .

Table 4.1: Design Parameters of Harmonic SAW Devices.

\begin{tabular}{ccccccc}
\hline $\begin{array}{c}\text { Harmonic } \\
\text { Mode }^{\mathrm{a}}\end{array}$ & $\begin{array}{c}\text { Finger } \\
\text { Width } \\
(\mu \mathrm{m})\end{array}$ & $\begin{array}{c}\text { Finger } \\
\text { Spacing } \\
(\mu \mathrm{m})\end{array}$ & $\begin{array}{c}\eta \\
(\%)\end{array}$ & $\begin{array}{c}\text { Number } \\
\text { of } \\
\text { Segments }\end{array}$ & $\begin{array}{c}\mathrm{S}_{n} \\
(\mu \mathrm{m})\end{array}$ & $\begin{array}{c}\mathrm{W} \\
(\mu \mathrm{m})\end{array}$ \\
\hline 0 & 8 & 8 & 50 & 1 & 1280 & 0 \\
0 & 5 & 5 & 50 & 1 & 160 & 0 \\
0 & 2 & 2 & 50 & 1 & 320 & 0 \\
5 & 5 & 5 & 50 & 5 & 140 & 4 \\
5 & 5 & 5 & 50 & 5 & 10 & 4 \\
7 & 6.5 & 3.5 & 65 & 7 & 115 & 2.857 \\
9 & 5 & 5 & 50 & 9 & 89 & 2.222 \\
11 & 6 & 4 & 60 & 11 & 73 & 1.818 \\
\hline
\end{tabular}

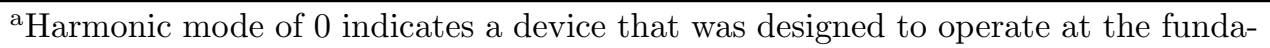
mental frequency.

\subsubsection{Harmonic SAW Device Frequency Response}

The wideband frequency response of five separate SAW devices designed to operate at the fundamental frequency, fifth, seventh, ninth and eleventh harmonics are shown in Figure 4.7. These frequency responses represent the response of a typical device measured for each harmonic mode. Devices designed to operate at the fundamental, fifth, seventh, ninth and eleventh harmonics have peaks at 230, 962, 1338, 1720 and $2100 \mathrm{MHz}$ respectively. Each peak is clearly visible in Figure 4.7; however, the increasing electromagnetic feedthrough (EF) at higher frequencies severely degrades the response of devices beyond the 7th harmonic. Devices designed to operate at harmonic frequencies dampen the fundamental response, which in turn, has the effect of drastically reducing the energy coupled to bulk modes. As seen in Figure 4.7, bulk modes appear in the frequency response of the device designed to operate at the fundamental from 800 


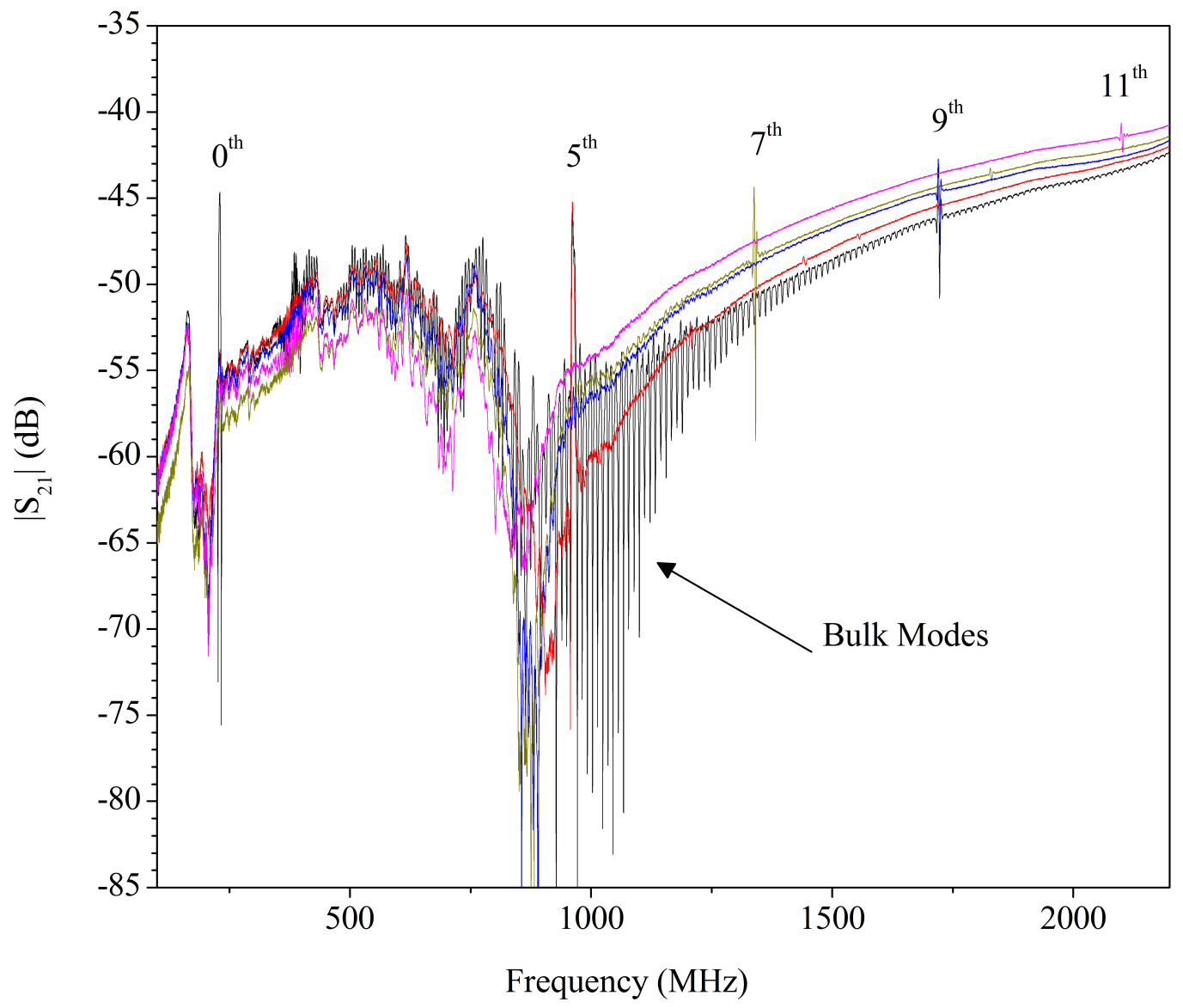

Figure 4.7: Wideband frequency response of five individual SAW devices. Devices were designed to operate at the fundamental, fitfh harmonic, seventh harmonic, nineth harmonic and eleventh harmonic [42].

$1200 \mathrm{MHz}$. These bulk modes are suppressed in all devices designed to operate at harmonic frequencies.

The local frequency response a device designed to operate at the fundamental frequency is compared to the local frequency response for devices designed to operate at each of the harmonics in Figure 4.8. In Figure 4.8(a), the response at the fifth harmonic for both devices is similar; however, the reduction of bulk modes for the harmonic device results in a $5 \mathrm{~dB}$ increase in the sideband rejection or signal to noise ratio (SNR). For the seventh and eleventh harmonic responses, shown in Figure 4.8(b) and 4.8(d) respectively, there is no response for a device designed to operate at the fundamental frequency, where $\eta=0.5$. This is because the relative harmonic efficiency for the seventh 


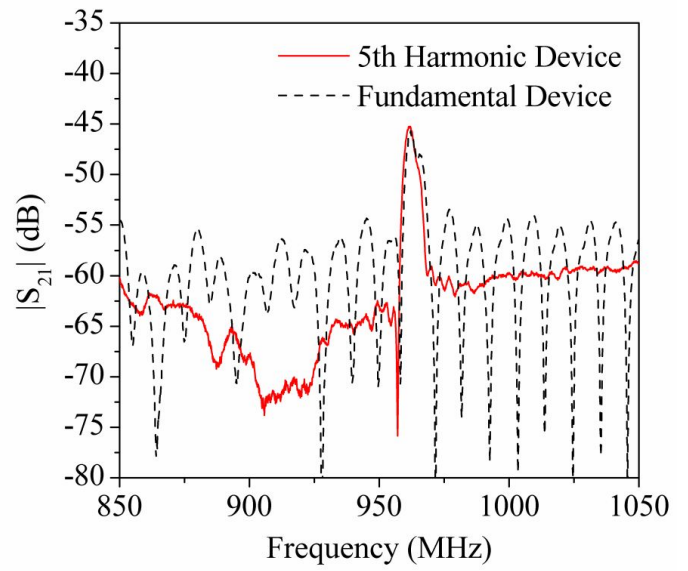

(a) Fifth Harmonic

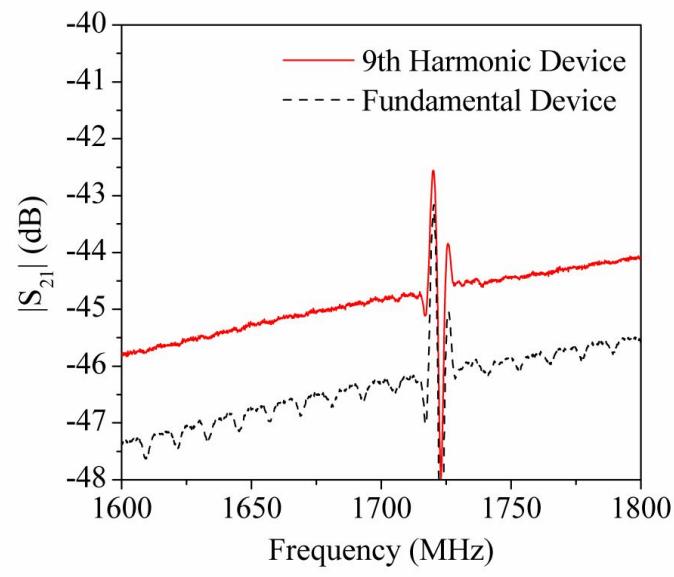

(c) Nineth Harmonic

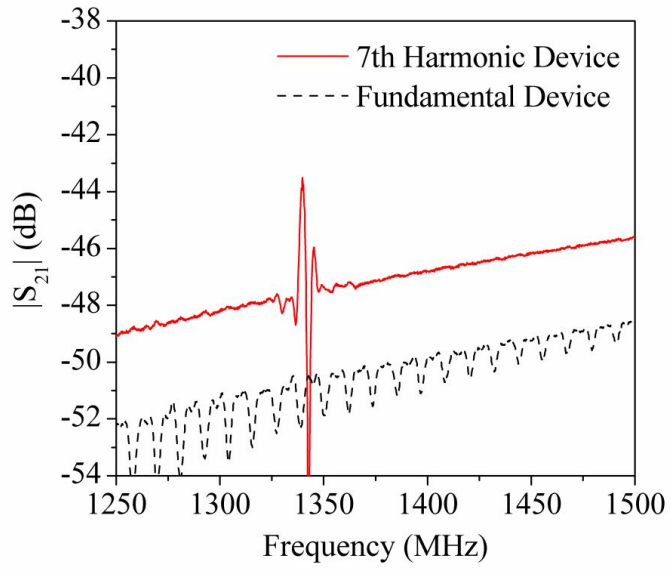

(b) Seventh Harmonic

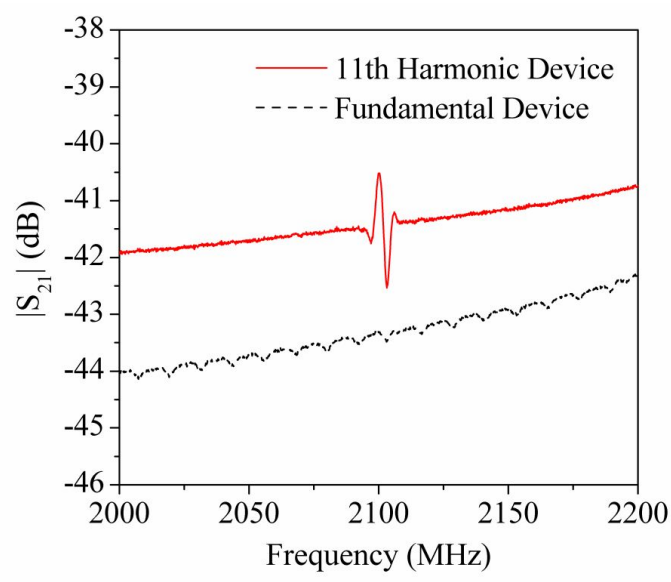

(d) Eleventh Harmonic

Figure 4.8: Comparison of the local frequency response of a SAW device designed to operate at the fundamental frequency with the frequency response of devices designed to operate at the (a) fifth, (b) seventh, (c) nineth and (d) eleventh harmonics [42].

and eleventh harmonic is zero for a metallization ratio $\eta=0.5$ [51]. There is no significant improvement in the ninth harmonic response of a device designed to operate at the ninth harmonic versus a device designed to operate at the fundamental frequency.

With the fundamental frequency at $230 \mathrm{MHz}$, and for an acoustically nondispersive material such as GaN, one expect the fifth, seventh, ninth and eleventh harmonic responses to occur at 1150, 1610, 2070 and $2530 \mathrm{MHz}$ respectively. As seen in 
Figure 4.7, this is not the case for SAW devices on GaN thin films on sapphire. This effect is covered in detail in the next section.

\subsection{Substrate Effects on Propagation of SAW in GaN}

In Figure 4.7, in the previous section, we saw that the harmonic frequencies are not what one would expect for SAWs traveling on a non-dispersive material, like GaN. The dispersion of acoustic waves on GaN in this study is due to the sapphire substrate. When the thickness of the GaN layer is thinner than the wavelength of the SAW, the acoustic velocity of the SAW is a combination of the acoustic velocity of the GaN and the sapphire substrate combined. Figure 4.9 shows the SAW dispersion in GaN thin films as a function of thickness.

There is also an anisotropic propagation due to the differences in acoustic properties with respect to the crystal orientation associated with the sapphire substrate in the c-plane [54]. This results in a different acoustic velocity and therefore a different

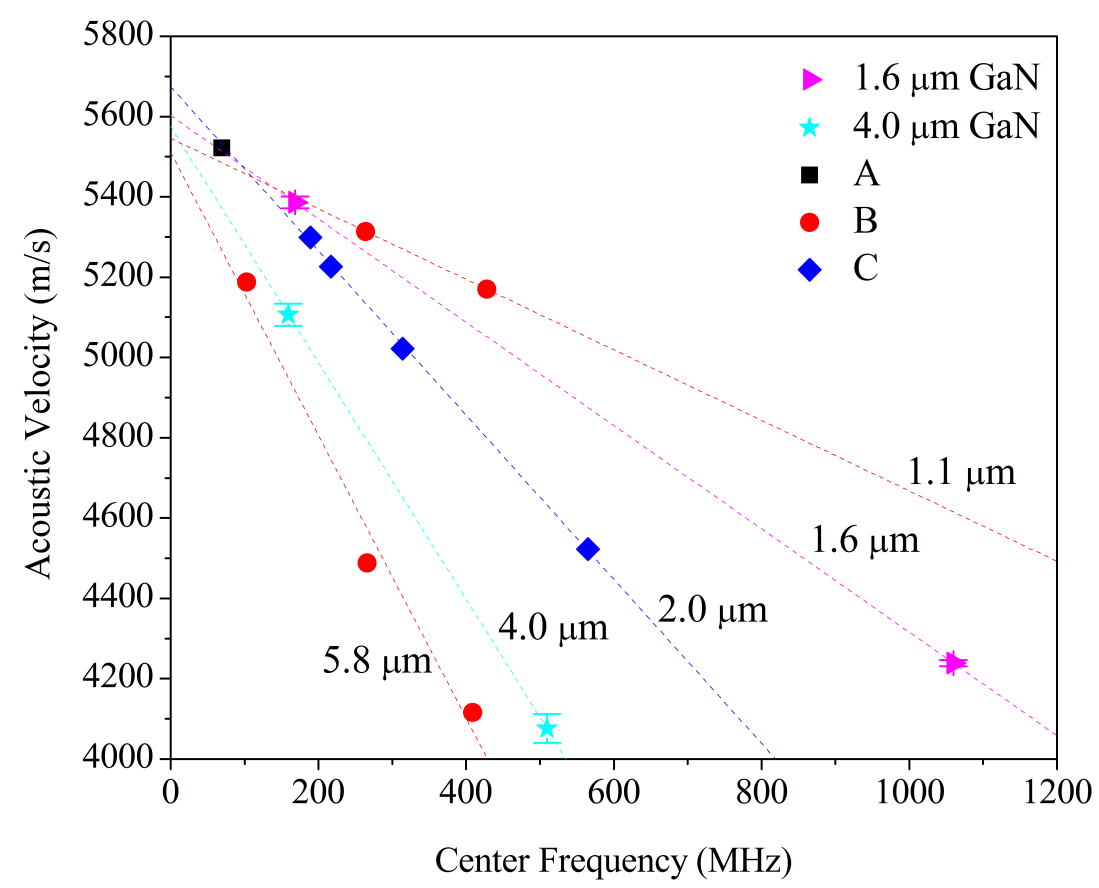

Figure 4.9: Dispersion of SAWs in GaN thin films wrt film thickness. A from [52], B from [53] and C from [44]. 


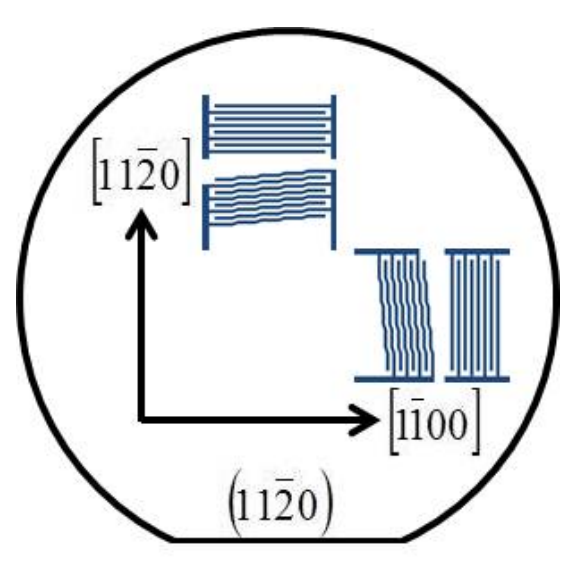

(a) Device orientation

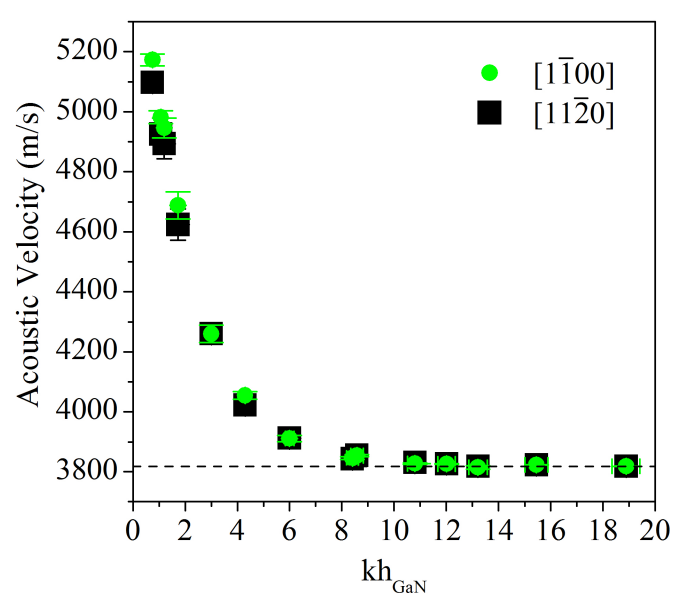

(b) Acoustic velocity vs. $k h_{\mathrm{GaN}}$

Figure 4.10: (a)Graphical representation of the orientation between SAW devices and the sapphire substrate and (b) $S A W$ dispersion as a funtion of $k h_{\mathrm{GaN}}$ for the different crystal orientations [42].

operating frequency for devices oriented differently on GaN/ sapphire when the SAW wavelength is longer than the GaN film thickness. To characterize this effect, devices were oriented parallel and perpendicular to the reference flat on the sapphire substrates. The reference flat is parallel to the plane, with devices being oriented along the and directions. Device orientation with respect to crystal directions is shown in Figure 4.10(a). The effect of device/substrate orientation on the acoustic velocity of SAW devices on GaN/sapphire with respect to $k h_{\mathrm{GaN}}$ is shown in Figure $4.10(\mathrm{~b})$.

For small $k h_{\text {GaN }}$ values, there is a difference of the acoustic velocity with respect to crystal orientation. The acoustic velocity approaches the acoustic velocity for the Rayleigh mode SAW in sapphire, which is reported in the literature as 5310 and 5486 $\mathrm{m} / \mathrm{s}$ for the and directions respectively [54]. When $k h_{\mathrm{GaN}}=6.28$, the GaN film thickness is equal to the wavelength, It can be seen in Figure $4.10(\mathrm{~b})$, that at this $k h_{\mathrm{GaN}}$ value and higher, there is no longer a separation of the measured acoustic velocities due to crystal orientation; however, there is still a decrease in the measured acoustic velocity as $k h_{\mathrm{GaN}}$ approaches 10. This suggests that even though the anisotropic effects of the substrate are negligible above $k h_{\mathrm{GaN}}=6.28$, there is still an effect of the substrate on the acoustic 
velocity up to a $k h_{\mathrm{GaN}}$ value of $\approx 10$. This is because, to a certain extent, the energy of a SAW extends further than one wavelength into the material. At $k h_{\mathrm{GaN}}=10$ and above the acoustic velocity of the Rayleigh mode SAW converges to $3820 \mathrm{~m} / \mathrm{s}$ and the effects of the sapphire substrate on the response of the SAW device become negligible.

For a device designed to operate on GaN at the fundamental frequency, with $\lambda_{0}=20 \mu \mathrm{m}$ and $\nu=3820 \mathrm{~m} / \mathrm{s}$, the GaN film thickness would need to be $31.83 \mu \mathrm{m}$ for $k h_{\mathrm{GaN}}=10$. Conversely, devices designed to operate at the 5th, 7th, 9th and 11th harmonics, with the same parameters, would only require a GaN film thickness of 6.37, 4.55, 3.54 and $2.89 \mu \mathrm{m}$ respectively. Devices could also be designed to operate with $\lambda_{0}$ $=10 \mu \mathrm{m}$, resulting in the reduction of the needed GaN thin film layer thickness by a factor of 2 and still not requiring sub-micron fabrication of IDTs. By designing devices to operate at higher harmonics, substrate effects can be eliminated with much thinner GaN films and larger IDTs, with process times being reduced because the need to align devices on samples is eliminated.

In addition to the above-mentioned advantages, operating at higher harmonics would also increase the sensitivity of a SAW sensor for a given IDT finger size. The sensitivity, S, given in equation 4.4, shows that the sensitivity of a SAW device is proportional to the square of the operating frequency. For devices operating at the 5th, 7 th, 9th and 11th harmonics, this would result in the increase of the sensitivity for a given SAW device by a factor of $25,49,81$, and 121 respectively.

\subsection{GaN Based SAW Devices for High Temperature Applications}

There are many materials that exhibit outstanding SAW characteristics at room temperature and were listed in Section 4.2. Langasite has been in the forefront of research for high temperature SAW materials because of its piezoelectric stability up to $1473{ }^{\circ} \mathrm{C}$ [55-64]. However, langasite exhibits large acoustic propagation losses at high temper- 


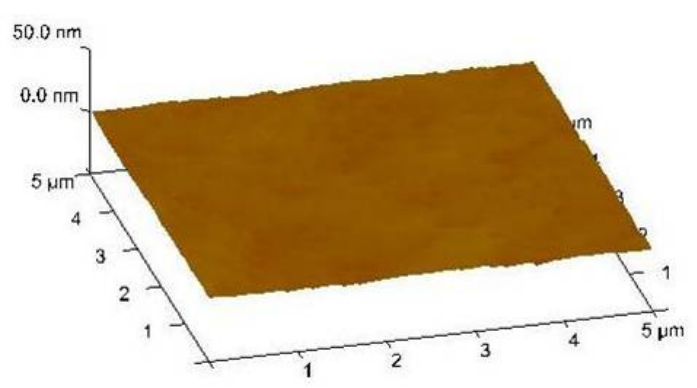

(a) Not Annealed

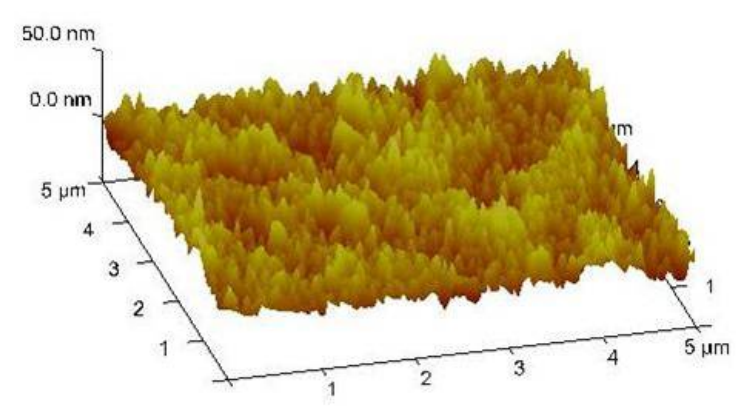

(c) $800^{\circ} \mathrm{C}$

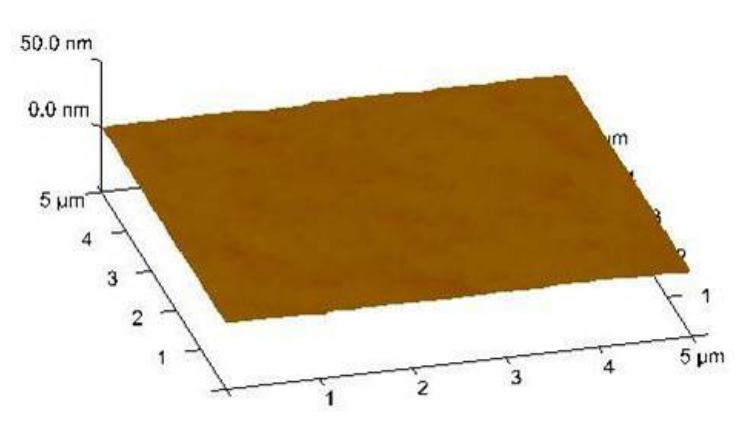

(b) $600^{\circ} \mathrm{C}$

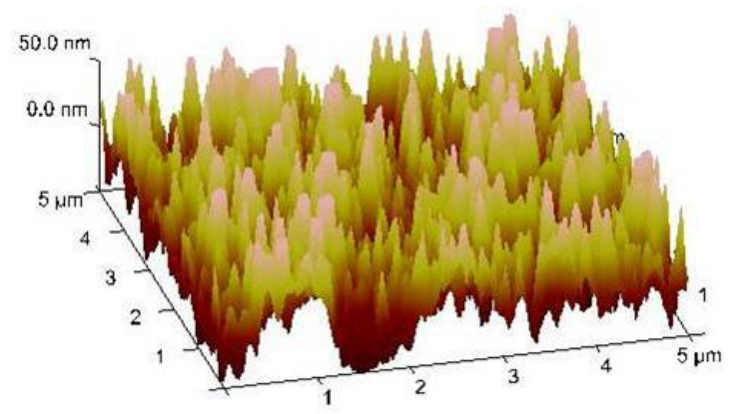

(d) $1000^{\circ} \mathrm{C}$

Figure 4.11: 3-D AFM scans of the GaN thin film surface (a) before annealing, and after annealing for 2 hours at (b) $600^{\circ} \mathrm{C}$, (c) $800^{\circ} \mathrm{C}$ and (d) $1000^{\circ} \mathrm{C}$ in atmosphere [65].

atures that increase with frequency, making it unsuitable for devices operating at high temperatures and high frequencies. Aluminum and gallium nitride have been considered for use in high temperature SAW devices, but very little experimental research has been conducted to confirm their piezoelectric and SAW characteristics at high temperatures.

For the work in this section, $4.0 \mu \mathrm{m}$ GaN thin films were grown on sapphire substrates, as described in Section 3.1.1. Surface morphology of the as-grown GaN thin films was characterized using AFM and oxygen content was measured using EDS. Samples were then thermally cycled in an atmospheric environment at temperatures ranging from 450 to $1000{ }^{\circ} \mathrm{C}$ in a Thermolyne 6000 series furnace. Thermal cycling involved slowly raising the sample temperature to the target temperature, holding the sample at the target temperature for a period of two hours, and then gradually cooling 


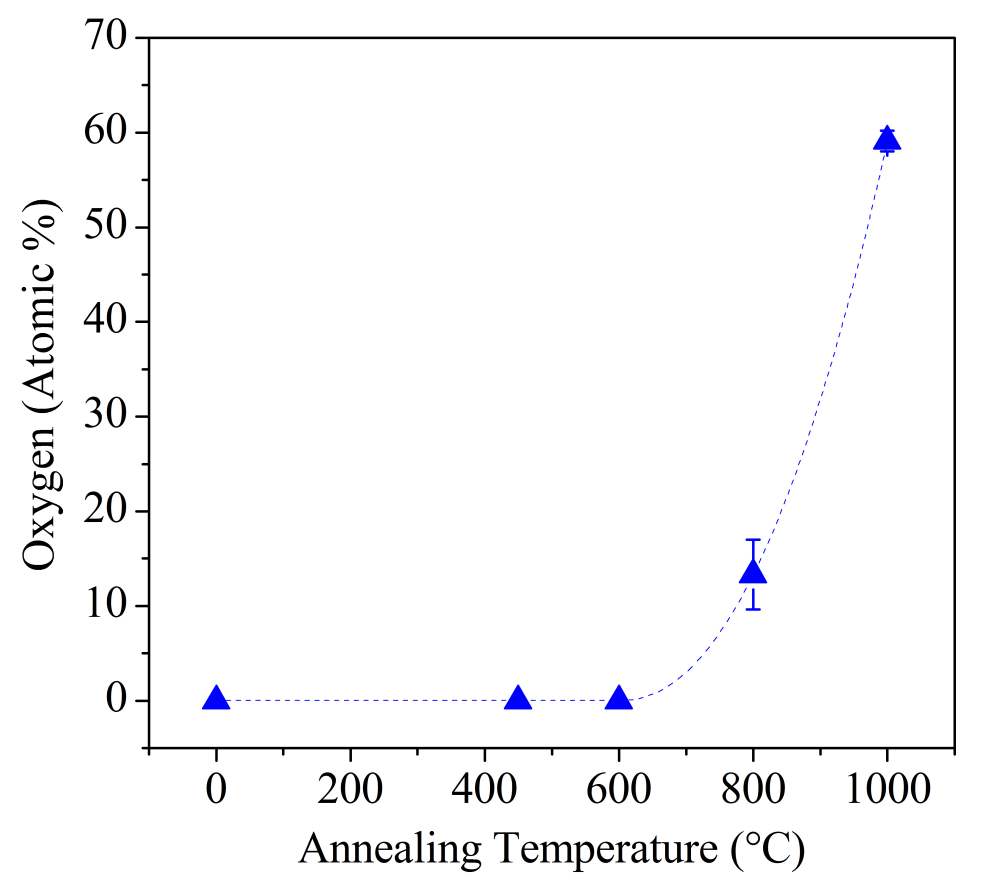

Figure 4.12: Oxygen content of GaN thin films as measured by energy dispersive X-ray spectroscopy (EDS) [65]. Dashed line serves as a guide for the eye and is not a curve fit to the data.

the sample to room temperature over a period of 6 to 12 hours. The slow heating and cooling of the samples was done to eliminate thermal shock as a possible cause for any difference noted in measurements before and after annealing.

The 3-D AFM plots of the GaN samples are shown in Figure 4.11. The RMS surface roughness of the as-grown GaN thin films was on the order of $1 \mathrm{~nm}$. The surface roughness was unchanged after annealing at $600{ }^{\circ} \mathrm{C}$ and began to degrade after annealing at $800{ }^{\circ} \mathrm{C}$, where the roughness was measured to be $8 \mathrm{~nm}$. The GaN surface degraded rapidly after annealing at $1000{ }^{\circ} \mathrm{C}$ and the RMS surface roughness was found to be greater then $25 \mathrm{~nm}$. A $25 \mathrm{~nm}$ RMS surface roughness is small compared to the wavelength of SAW devices with $\lambda_{0}$ on the order of $\mu \mathrm{m}$. However, even this small change could have drastic effects on the piezoelectric coupling coefficient and performance of SAW devices.

For the as-grown GaN films, no oxygen was detectable by EDS, as shown in Figure 4.12. Care was taken to ensure the electron beam did not penetrate all the way 


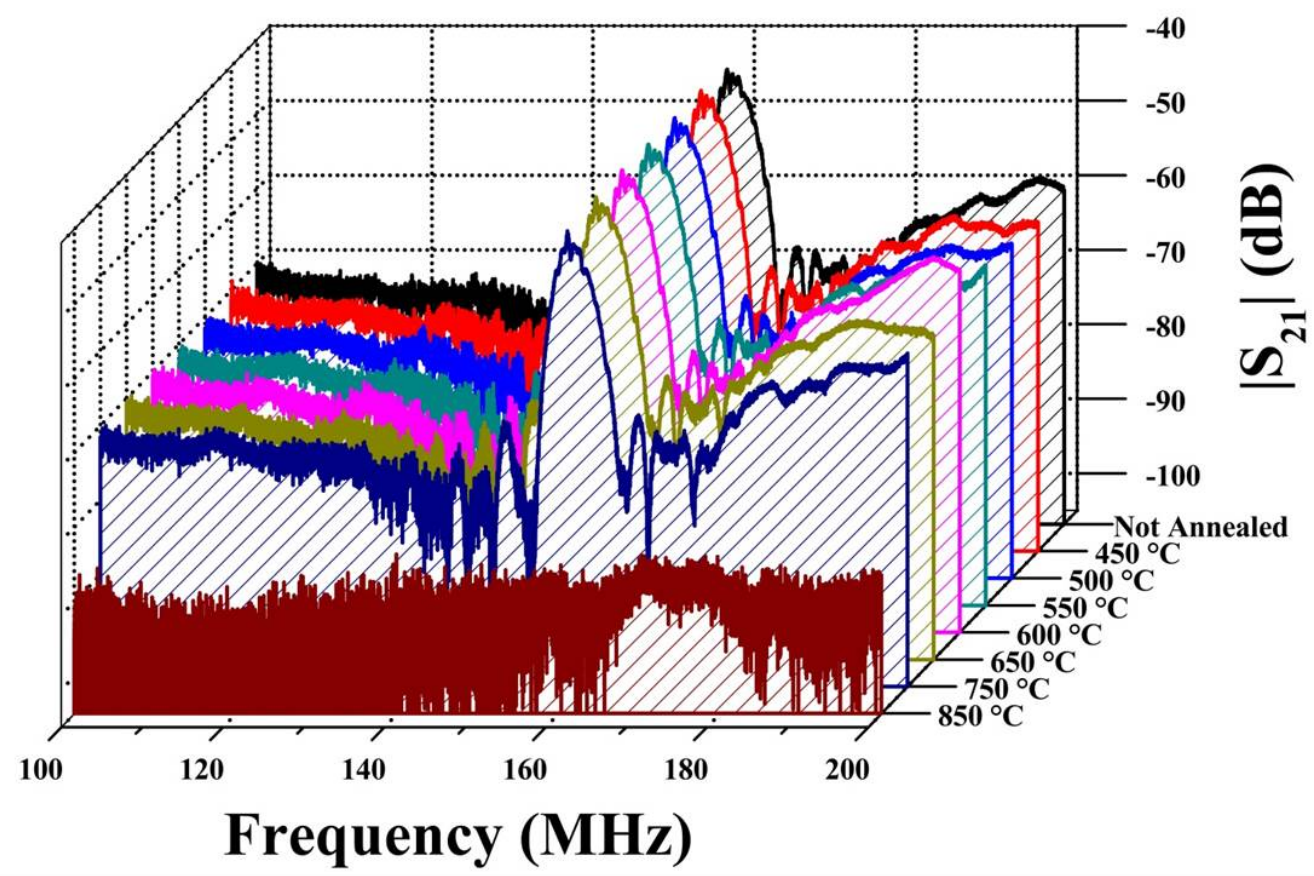

Figure 4.13: Frequency response of a thermally cycled GaN based SAW device.

to the substrate, minimizing any false oxygen detection from sapphire. Oxygen was still not present at any detectable level in the samples after annealing at $600{ }^{\circ} \mathrm{C}$. At $800{ }^{\circ} \mathrm{C}$ the samples showed moderate oxidation. After annealing at $1000^{\circ} \mathrm{C}$, the $\mathrm{GaN}$ films were completely oxidized and nitrogen was no longer detectable by EDS (nitrogen scans not shown).

For this study, a direct measurement of the piezoelectric coefficients of GaN was not made. To validate that the piezoelectric properties did not degrade after thermal cycling, SAW devices were fabricated on new GaN samples. The frequency response of the un-annealed sample was measured for reference. The samples then underwent the same thermal cycling mentioned above, but at smaller intervals of temperature. The frequency repsonse of the samples was measured after each subsequent annealing. The results of a single device is shown in Figure 4.13, and showed a typical response similar to all devices measured.

SAW devices did not show reduced response until annealing at $750{ }^{\circ} \mathrm{C}$, at which 


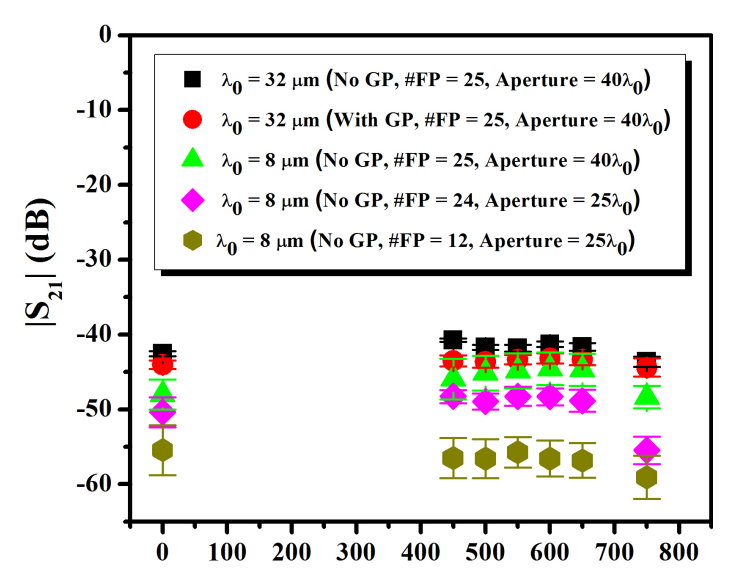

Annealing Temperature $\left({ }^{\circ} \mathrm{C}\right)$

(a) Insertion Loss

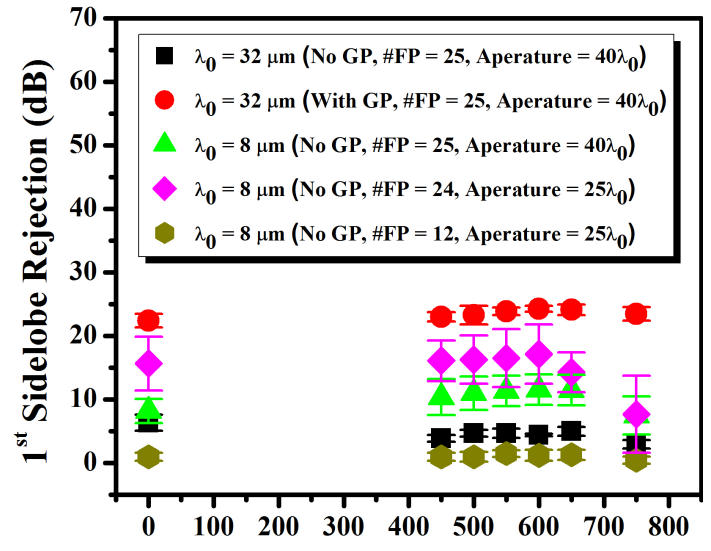

Annealing Temperature $\left({ }^{\circ} \mathrm{C}\right)$

(b) Signal to Noise Ratio

Figure 4.14: (a)Insertion loss at the operating frequency as a function of annealing temperature and (b) the signal to noise ratio as a function of annealing temperature [65].

point, insertion loss began to increase and the first sidelobe rejection began to decrease as shown in Figure 4.14. Samples were annealed at $850{ }^{\circ} \mathrm{C}$ and device response was completely lost, therefore, there was no data point measured for $850{ }^{\circ} \mathrm{C}$. A test setup would need to be consturcted to measure the in situ frequency response of SAW devices to verify operation while at elevated temperatures.

\subsection{GaN Based SAW Devices for Continous Emis- sions Monitoring}

In 1963, the United States government passed the Clean Air Act which launched research efforts to determine regulations that would protect the citizens from hazardous gases. In 1967, the Air Quality Act expanded those research efforts, and amendments to the Clean Air Act in 1970 set the first federal and state requirements for monitoring and regulating emissions in the US. The 1970 amendment also included mobile sources of emissions, including motor vehicle and airplanes. The last amendment to the Clean Air Act was in 1990 and included provisions for monitoring and controlling acid rain $[66,67]$.

In response to legislation, considerable research has been conducted which has 
resulted in continuous emissions monitoring (CEM) systems for stationary sources of hazardous gases, such as industrial plants [68-73]. These systems monitor emissions 24/7, 365 days a year. They are typically very large and expensive to operate, making them impracticable or impossible for use on mobile sources of emissions. Mobile source standards, like those for motor vehicles, only require an emissions standard at the time of production. After that, only the CARB states* have requirements for yearly smog checks of motor vehicles. Currently, for the majority of the US, there is no monitoring or regulation of emissions on motor vehicles after they are manufactured.

The most commonly used technology is based on electrochemical sensors. These generally resemble a fuel cell in their operation. However, the sensing device is not directly exposed to the exhaust flow. Gas detection is a two-stage process where the exhaust gas passes through a porous membrane to reach the sensing parts, and their lifetime will be shortened in very hot areas. Fiber optic technology has also been studied for exhaust gas monitoring [74-77]. Here, operation is based on optical absorption of light by gas species at characteristic wavelengths. Optical fiber sensors have some advantages such as freedom from electromagnetic and radio frequency interference. However, they are costly and the detection systems may be complex. Other available gas sensors used by automotive industries include catalytic combustion, field effect and IR devices. However, poor gas selectivity is evident in these technologies and their performance can degrade significantly due to dust and dirt.

Surface acoustic wave (SAW) sensors are superior in regards to their sensitivity when compared to other conventional sensor technologies [5]. In this work, SAW devices on GaN thin films are demonstrated as an excellent candidate for small, lightweight, relatively inexpensive sensors for real-time chemical gas monitoring in exhaust systems. The engine used was a 1992 Detroit Diesel Series 60, which can be seen in Figure 4.15 and the fuel type was ordinary no. 2 on-road diesel. Figure 4.16 shows a sample

\footnotetext{
*Those that follow the more strict standards set forth by the California Air Resources Board.
} 


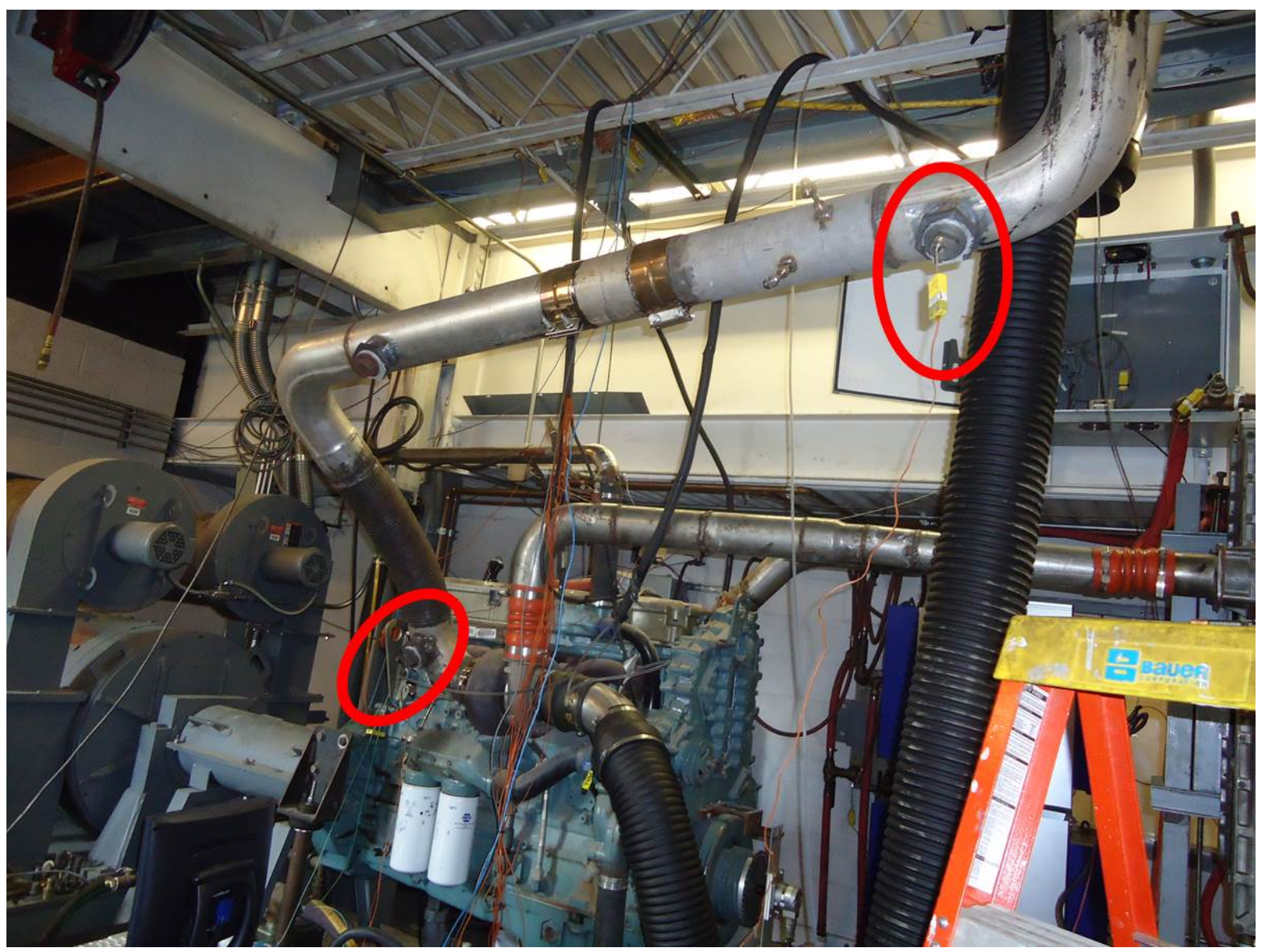

Figure 4.15: Real world exhaust engine test setup. Red circles indicate where SAW device samples were inserted into the exhaust stream.

with GaN based SAW devices mounted on a custom sample holder with an integrated thermocouple. The sample holder is loaded into the exhaust pipe, indicated by red circles in Figure 4.15. The engine was controlled by a dynamometer and local exhaust temperature at the SAW devices and concentration of chemical species was recorded. Samples were then cleaned in acetone and devices were re-measured with the VNA. This experiment was cycled four times. A list of the test parameters can be found in Table 4.2, and a plot of a typical engine test can be found in Figure 4.17.

The performance of SAW devices was recorded after each exhaust test through the measurement of four main quantities; insertion loss (IL), signal to noise ratio (SNR), operating frequency $(\mathrm{OF})$ and quality factor $(\mathrm{QF})$. The quality factor is defined as the 


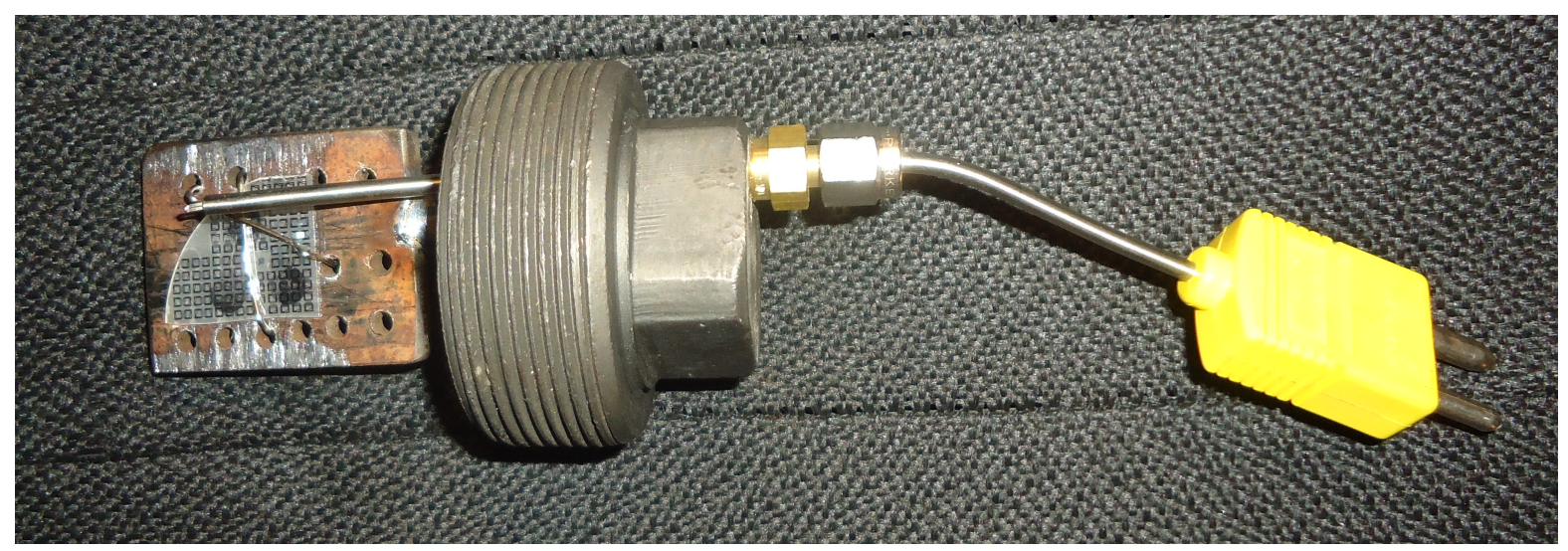

Figure 4.16: A sample with fabricated SAW devices mounted for insertion into the exhaust stream of the test engine. Holder is equipped with a thermocouple to record the local temperature during test runs.

Table 4.2: Engine Exhaust Test Parameters.

\begin{tabular}{llllllll}
\hline $\begin{array}{l}\text { Exhaust } \\
\text { Test No. }\end{array}$ & $\begin{array}{l}\text { Duration } \\
(\mathrm{Hrs})\end{array}$ & $\begin{array}{l}\mathrm{Max} \\
\text { Temp. } \\
\left({ }^{\circ} \mathrm{C}\right)\end{array}$ & $\begin{array}{l}\mathrm{Avg} \\
\text { Temp. } \\
\left({ }^{\circ} \mathrm{C}\right)\end{array}$ & $\begin{array}{l}\mathrm{HC} \\
(\mathrm{ppm})\end{array}$ & $\begin{array}{l}\mathrm{NO}_{x} \\
(\mathrm{ppm})\end{array}$ & $\begin{array}{l}\mathrm{CO}_{x} \\
(\mathrm{ppm})\end{array}$ & $\begin{array}{l}\mathrm{CO} \\
(\mathrm{ppm})\end{array}$ \\
\hline 1 & 1 & 448 & 370 & 8 & 224 & 1500 & 97 \\
2 & 1 & 465 & 220 & $\mathrm{NR}$ & $\mathrm{NR}$ & $\mathrm{NR}$ & $\mathrm{NR}$ \\
3 & 2 & 459 & 216 & $\mathrm{NR}$ & $\mathrm{NR}$ & $\mathrm{NR}$ & $\mathrm{NR}$ \\
4 & 2 & 464 & 272 & 12 & 155 & 9500 & 20 \\
\hline
\end{tabular}

NR represents a value that was not recorded during testing.

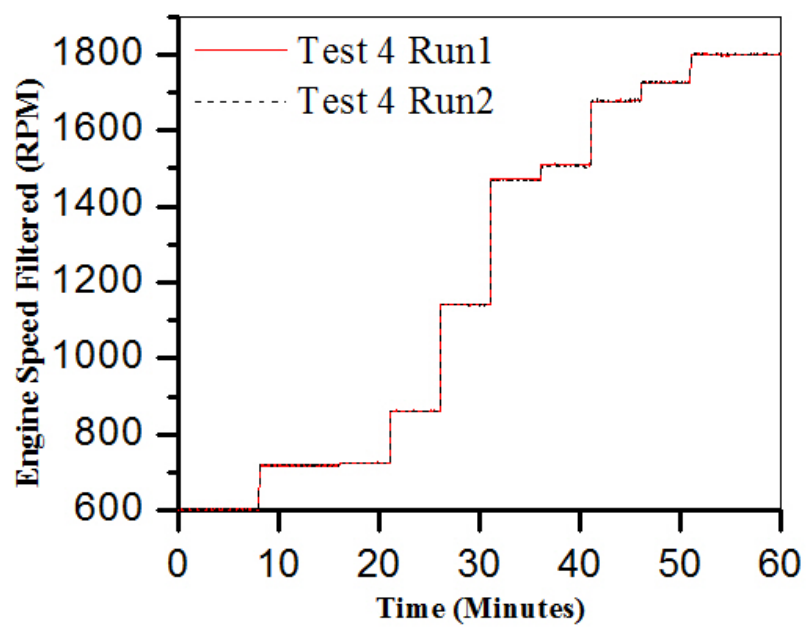

Figure 4.17: Typical RPM profile for an engine exhaust test. Test pattern was repeated for tests longer then one hour. 


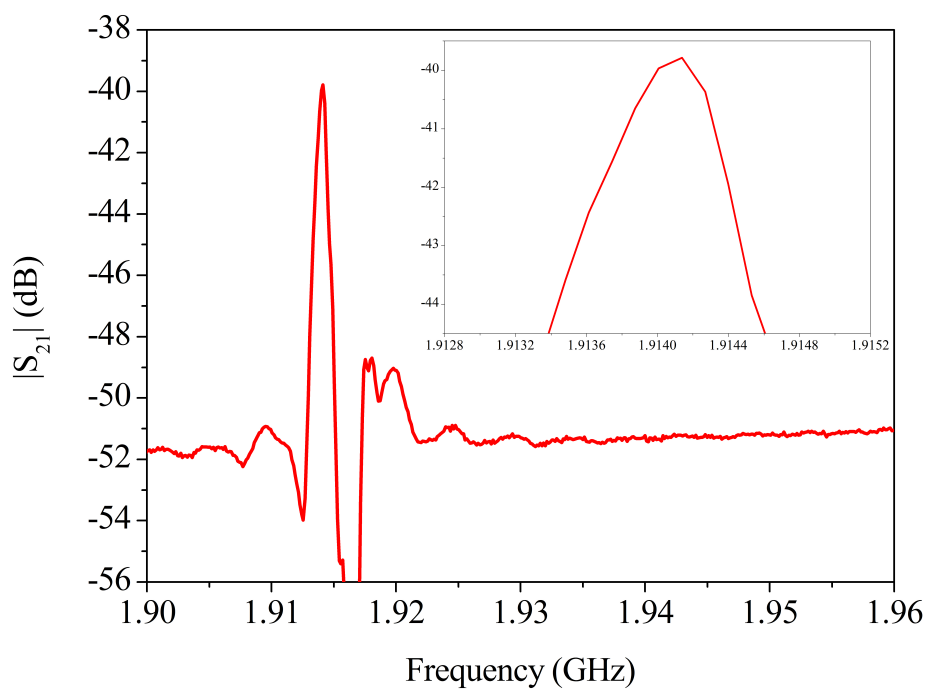

Figure 4.18: Local frequency response of a SAW device designed to operate at the seventh harmonic with $\eta=0.35$. The quality factor of this device is $\approx 2423$.

ratio between the operating frequency and the full width half maximum (FWHM). A high quality factor implies a very sharp response with narrow FWHM. The desired properties of a SAW device response are dependent on the device application. For bandpass filtering, the FWHM is engineered to the specific range of frequencies of interest. For sensor applications, the most common method is to measure the shift in the operating frequency as the surface chemistry changes in response to interacting with the target gas species. Higher operating frequencies are desired to increase the sensitivity of the device, while a high quality factor is desired for higher resolution detection of the shift in operating frequency. Figure 4.18 shows the local frequency response of a seventh harmonic device with a quality factor measurement of about 2423. This is quite good for a SAW device, but still low when compared to crystal resonators, which have QFs on the order of 10000 and higher.

Many different devices were measured that each had unique performance parameters based on IDT geometry, location on substrate, IDT metal, etc. For this reason, measurements were normalized by dividing each recorded value after an exhaust test by its initial value. All normalized values for a given harmonic were then averaged. In this 


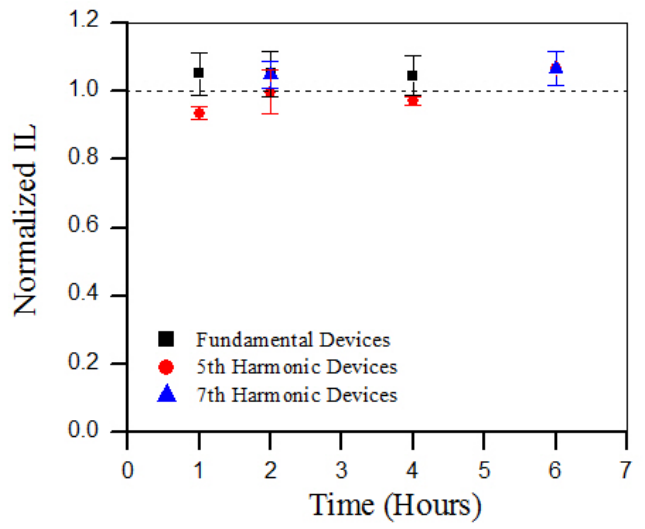

(a) Insertion Loss

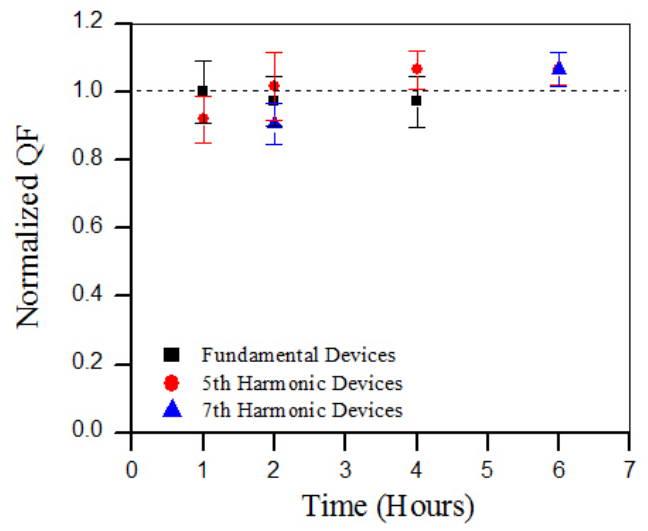

(c) Quality Factor

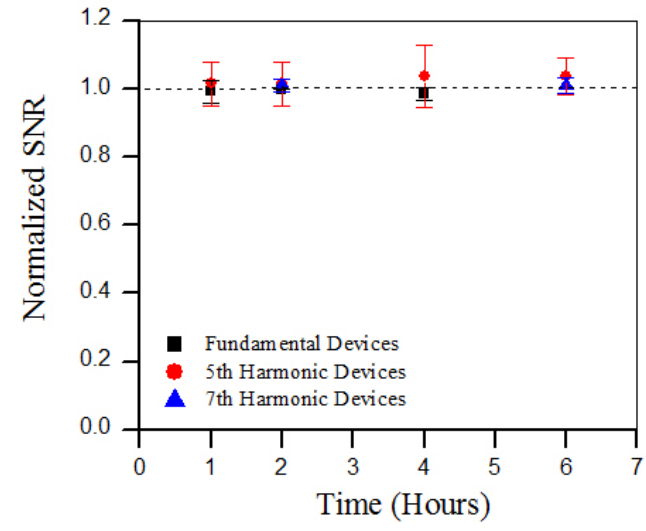

(b) Signal to Noise Ratio

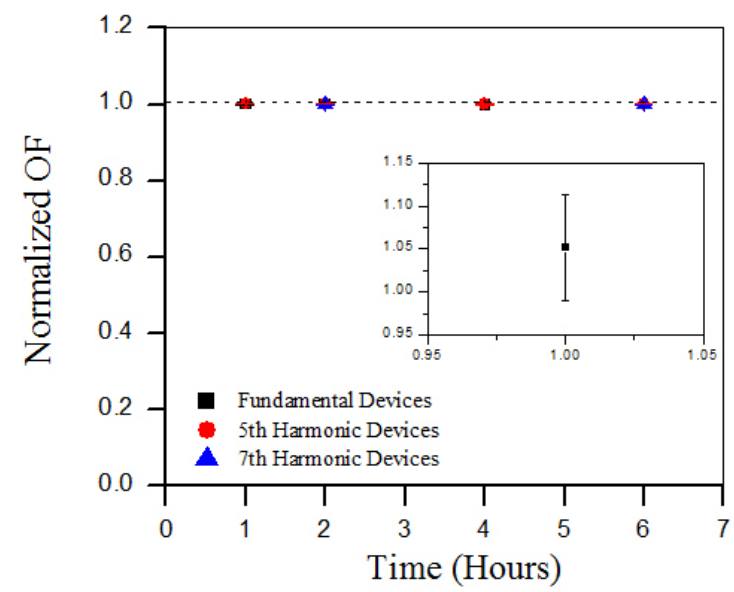

(d) Operating Frequency

Figure 4.19: Normalized measured values for (a) insertion loss (IL), (b) signal to noise ratio, (c) quality factor $(Q F)$ and (d) operating frequency (OF) [78]. 
way, only the change in device performance before and after each exhaust test is monitored. Figure 4.19 shows the normalized values for each type of measurement after each test. A normalized value of 1.0 indicates no change in that device parameter before and after testing. A normalized IL value greater than 1.0 indicates performance degradation since a higher insertion loss indicates either lower electromechanical coupling between the voltage signal and the SAW, dampening of the SAW due to an increase in surface density or more scattering of the SAW due to cracking, increased surface roughness, etc. Figure 4.19(a) indicates an initial decrease in IL. This is most likely due to a slight smoothening of the surface with initial annealing of the samples at higher temperatures during exhaust testing. The IL may have increased slightly after six hours of testing. Figure 4.19(b) shows no statistical change in the measured SNR throughout the tests. Figure 4.19(c) also shows that the QF did not degrade and may have even improved slightly during testing. For devices to act as sensors, where the shift in operating frequency is measured, Figure $4.19(\mathrm{~d})$ is of the most interest. In this plot, no change in operating frequency is measured. It is very important to note, however, that the frequency response was measured over a wide range of $10 \mathrm{MHz}$ to $2.2 \mathrm{GHz}$ and the resolution of each measurement was about $130 \mathrm{kHz}$. Therefore, it can only be stated that the frequency of devices shifted less than $130 \mathrm{kHz}$. More exhaust tests are needed with higher resolution measurements to identify if there is any shift in operating frequency below $130 \mathrm{kHz}$.

\subsection{Electromagnetic Feedthrough}

The reduction in SNR ratio at higher frequencies is attributed to electromagnetic feedthrough $(\mathrm{EF}) . \mathrm{EF}$ is the direct coupling of electromagnetic energy between input and output IDT due to the inherent capacitance associated with SAW devices. Time-domain gating can be used to remove the effects of electromagnetic feedthrough from the device response in post-measurement data processing. However, it is desirable to eliminate as much EF 


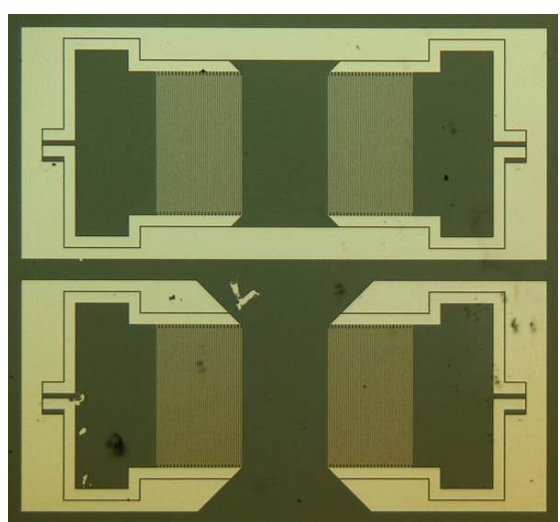

(a) Optical image of $S A W$ devices.

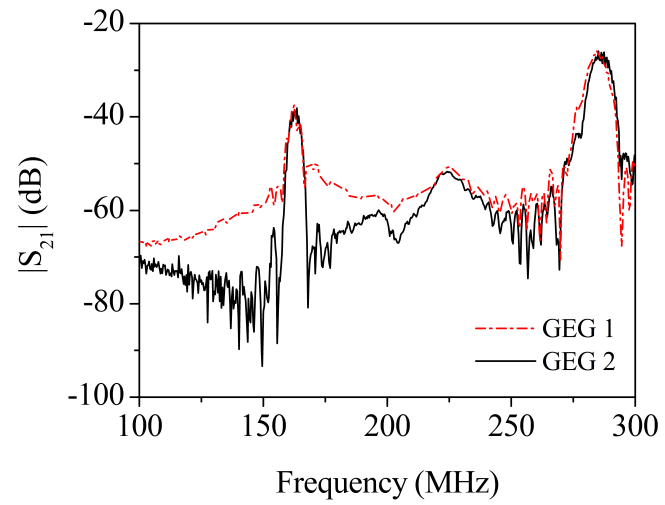

(b) Frequency response of $S A W$ devices.

Figure 4.20: Optical image showing the same SAW device with different ground electrode geometries $(G E G)(a)$ and their respective frequency responses (b). GEG 1 corresponds to the bottom geometry in (a) and the red dash-dot-dash curve in (b).

as possible through device design and packaging.

The authors have previously shown that EF can be reduced through different ground electrode geometries (GEG) for devices operating at the fundamental frequency [10]. This approach was applied to harmonic devices and it was found that different GEG configurations are needed to improve performance at harmonic frequencies with results

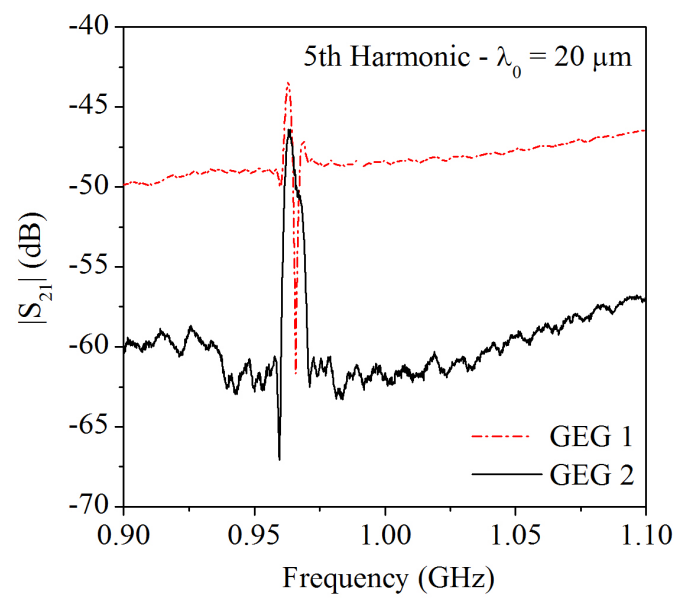

(a) Fifth Harmonic

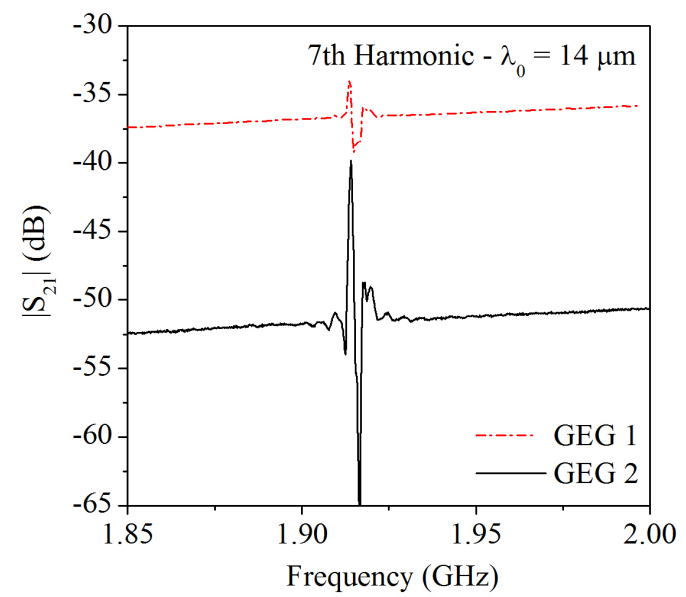

(b) Seventh Harmonic

Figure 4.21: Rayleigh mode frequency response of (A) fifth and (B) seventh harmonic SAW devices with different ground electrode geometries (GEG) [26]. GEG are simliar to the ones shown in Figure 4.20(a). 
shown in Figure 4. This improvement in device performance was typical of all devices measured. Different GEG need to be explored to see if even greater improvements can be achieved. This technique has not yet been applied to 9th or 11th harmonic devices, but the authors expect the same improvements in overall device response. 


\section{Chapter 5}

\section{High Electron Mobility Transistors}

\subsection{Introduction}

Since the introduction of the solid-state transistor in the 1940s [80], considerable research has been dedicated to improving device speed. Carrier mobility within a semiconductor material plays a significant role in overall device speed [81]. The necessity to dope semiconductors leads to ionized impurities that act as carrier scattering centers and drastically reduce the mobility from that of the intrinsic crystal [82]. In short, what makes a semiconductor device work also reduces its quality. Homojunction devices made of germanium or silicon dominated early transistor technology, and the reduced mobility

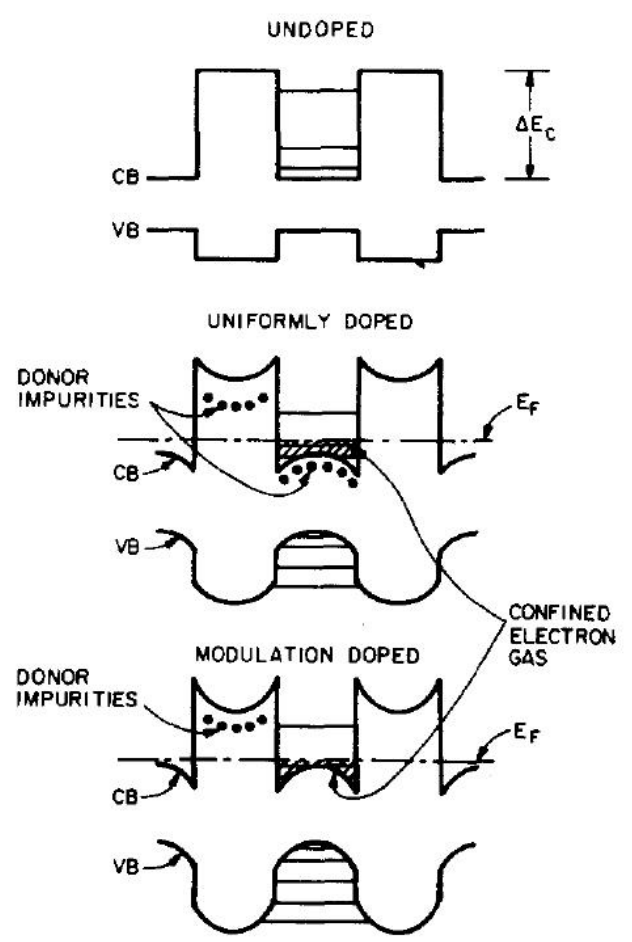

Figure 5.1: Schematic showing energy band diagrams for undoped, uniformly doped and modulation doped $A l_{x} G a_{1-x} A s /$ GaAs superlattices. Image from [79]. 
from doping was simply accepted as an unavoidable tradeoff for device fabrication.

In the late 1970s, with advances in the epitaxial growth of compound semiconductors, a modulation doping scheme was developed by R. Dingle et al. [79]. Modulation doping is the growth of a heterojunction where only the wider bandgap material is doped. Due to the band alignment and difference in the Fermi levels, band bending occurs at the junction. Therefore, it is possible to spatially separate carriers from their ionized impurities in an irreversible manner, which is shown in Figure 5.1. A 2-dimensional electron gas (2DEG) is formed in the undoped material and the mobility approaches that of the intrinsic semiconductor, and is significantly higher than that for a bulk semiconductor with the same doping concentration [83].

During the 70s and 80s, significant advances were made in modulation doping of the GaAs family of material [84]. In addition to increased mobility, there is no carrier freeze-out at low temperatures in modulation-doped heterostructures, like there is in bulk semiconductors [85]. This allows devices to operate at extremely low temperatures. In 1989, Pfeiffer et al. reported low temperature electron mobilities in GaAs on the order of $10^{7} \mathrm{~cm}^{2} /(\mathrm{V} \cdot \mathrm{s})[80]$.

The techniques used in an attempt to define the Hamiltonian in the Schrödinger equation include tight-binding-expansion [86], $\mathrm{k} \cdot \mathrm{p}$ expansion[87] and the effective mass model [88]. In 1986, Wong et al. developed a theoretical model to predict the electron concentrations and electronic structure of modulation-doped $\mathrm{GaAs} / \mathrm{Al}_{x} \mathrm{Ga}_{1-x} \mathrm{As}$ heterojunctions [81]. They used a psuedopotential model and found a simple perturbation model based on the effective mass envelope function model to be sufficient. They also investigated structures with large periodicity and considered the effects of long-range alloy ordering. Figure 2 shows the theoretical calculation for the charge densities in modulation-doped GaAs $/ \mathrm{Al}_{x} \mathrm{Ga}_{1-x} \mathrm{As}$ heterostructure. Here only the AlGaAs layer is doped, but it is clear that the electron concentration for the $\Gamma_{1}$ and $\Gamma_{2}$ state is primarily 
in the GaAs layer.

As demand for higher frequencies and increased power density transistor devices continues to drive production, new materials are being investigated [90]. Gallium nitride $(\mathrm{GaN})$ has emerged as the frontrunner to replace silicon and GaAs as the semiconductor of choice for high power high frequency applications [91]. Modulation doped $\mathrm{Al}_{x} \mathrm{Ga}_{1-x} \mathrm{~N}$ / GaN heterostructures have been extensively studied in the last two decades. The theoretical modeling of the GaN family of material is made more difficult due to the piezoelectric polarization and spontaneous polarization these materials exhibit. Morkoç et al., have developed a simple effective mass approximation to determine the electronic structure and electron density distribution in modulation doped $\mathrm{Al}_{x} \mathrm{Ga}_{1-x} \mathrm{~N} / \mathrm{GaN}$ heterostructures [89]. The calculated conduction band edge for a GaN based MODFET structure is shown in Figure 5.3. It is clear that the piezoelectric and spontaneous polarizations have a dramatic effect on the band structure. It is this change of the band

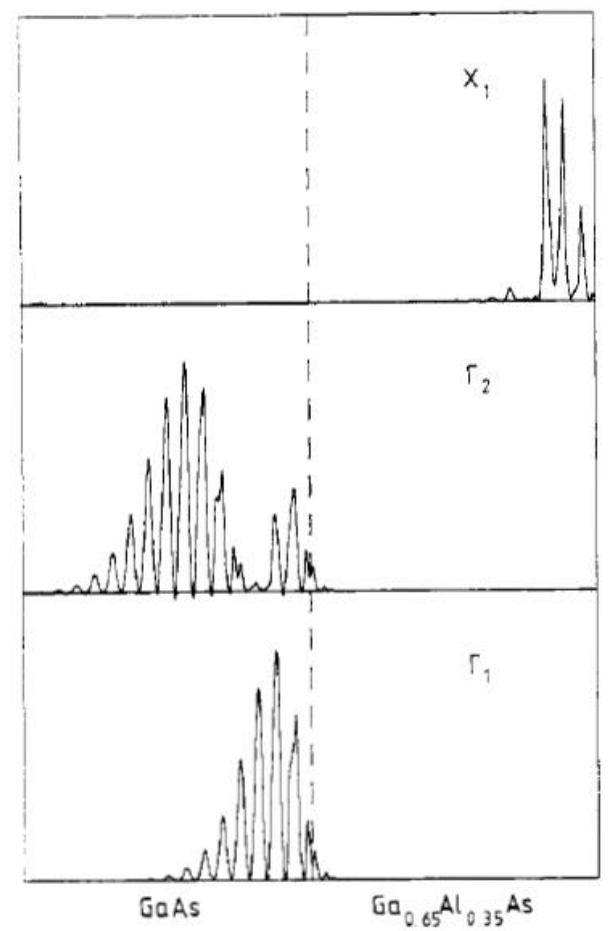

Figure 5.2: Theoretical calculation of charge densities in the modulation-doped $G a A s / A l_{x} G a_{1-x} A s$ heterostructure, from [81]. 
structure that results in the formation of a 2DEG in the $\mathrm{Al}_{x} \mathrm{Ga}_{1-x} \mathrm{~N} / \mathrm{GaN}$ heterostructure and is examined in detail in Section 5.2.

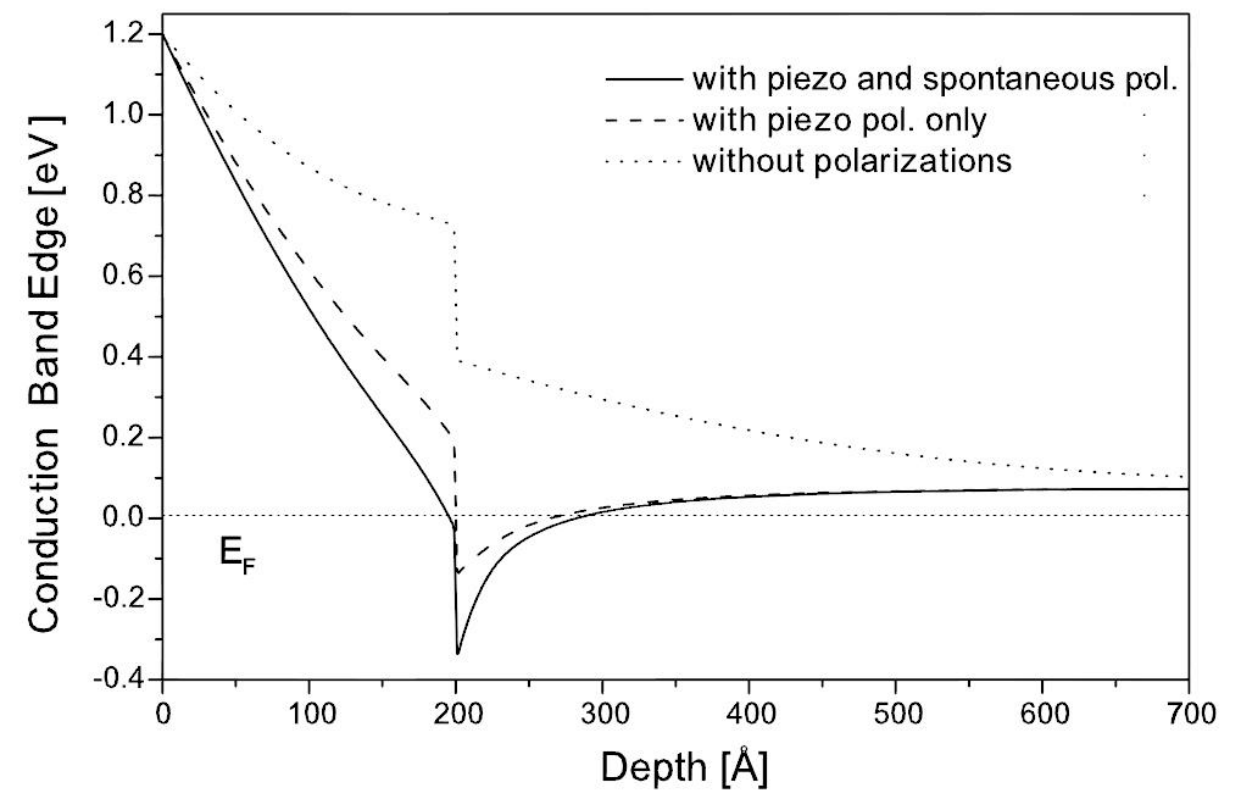

Figure 5.3: Conduction band edge for the nAlGaN/GaN MODFET structure, from [89]. 


\subsection{Theoretical Results}

The spontaneous and piezoelectric polarizations in the $\mathrm{Al}_{x} \mathrm{Ga}_{1-x} \mathrm{~N} / \mathrm{GaN}$ heterojunction can induce a 2DEG formation without the intentional doping of the semiconductor layers. In this work, the induced sheet charge carrier concentration resulting from this macroscopic polarization field is theoretically calculated. Figure 4 shows a basic schematic of a processed $\mathrm{Al}_{x} \mathrm{Ga}_{1-x} \mathrm{~N} / \mathrm{GaN}$ HEMT structure. In this work, only the $\mathrm{Al}_{x} \mathrm{Ga}_{1-x} \mathrm{~N}$ and GaN layers are considered, without the post processing etch and metalization.

Figure 5.4 shows the various possibilities and combinations of the spontaneous and piezoelectric polarizations. If the $\mathrm{Al}_{x} \mathrm{Ga}_{1-x} \mathrm{~N}$ layer is relaxed, only the spontaneous polarization exists. In general, the group III-Ns are grown in the wurtzite crystal structure, although it is possible for zincblende growth. This work focuses on the wurtzite structure and it is extremely important to note the asymmetry in this structure. The [0001] and [0001] directions are not equivalent. This results in a dependency of the polarization on the polarity of the grown films. The piezoelectric polarization occurs when one of the films is strained. Since AlN has a smaller a-lattice parameter than GaN, when GaN is relaxed, the AlGaN layer is under tensile strain, and when the AlGaN is relaxed, the GaN layer is under compressive strain. For this work, only the case of Gaface polar growths were the AlGaN layer is strained is considered. For MOVPE growth of GaN on sapphire, the Ga-face polarity is most common and for thin AlGaN grown on a thick GaN layer, AlGaN will be under tensile strain until it relaxes. Determining GaN polarity and AlGaN strain is not simple experimentally. However, a smooth, crack free surface is a very good indicator of Ga-face polarity and an AlGaN strained layer. For the following simulations, all material parameters, except bandgap, are assumed to be linear from GaN to AlN and can be found in [92]. The below equations can also be found in [92]. 


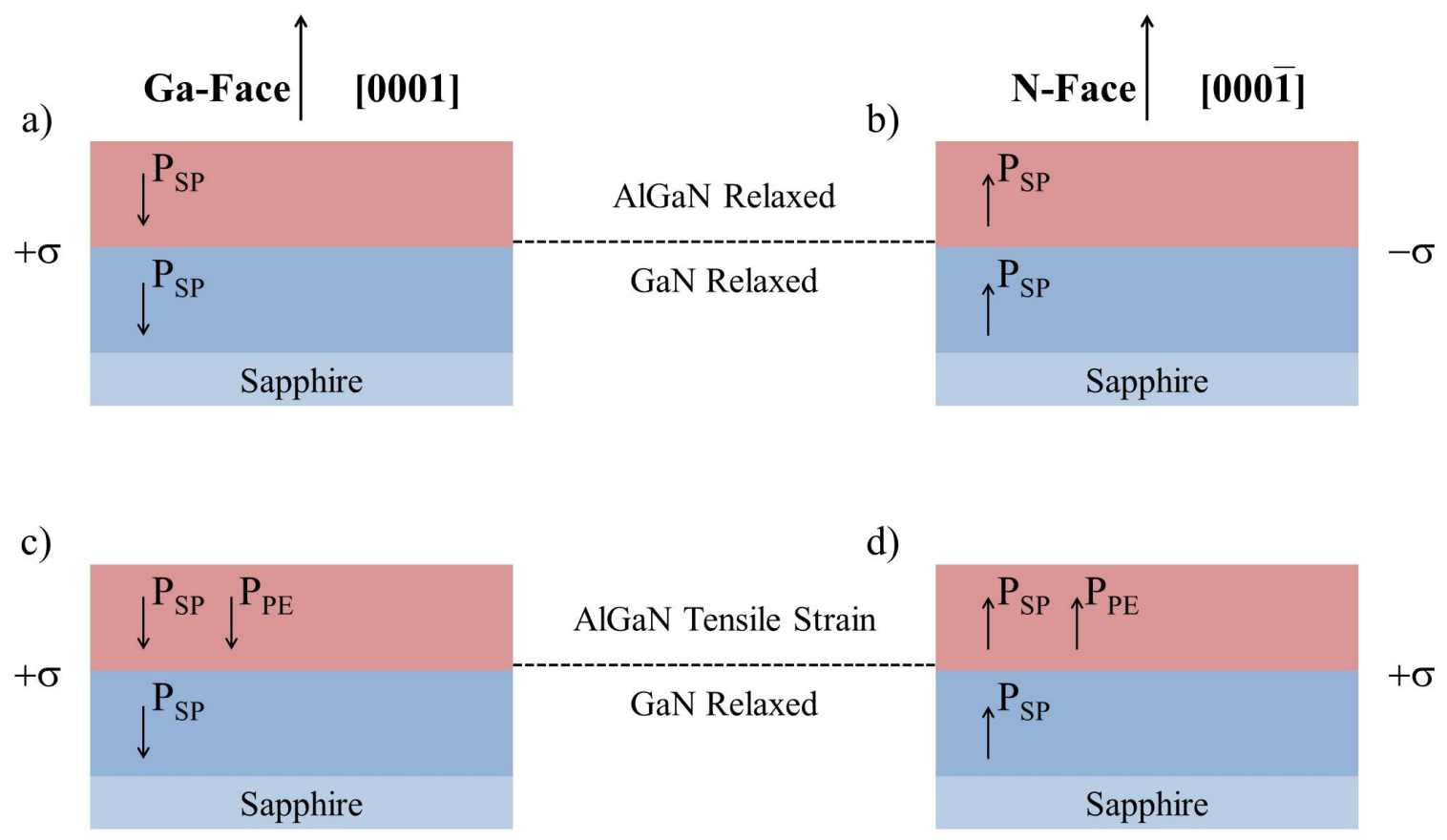

e)

$-\sigma$

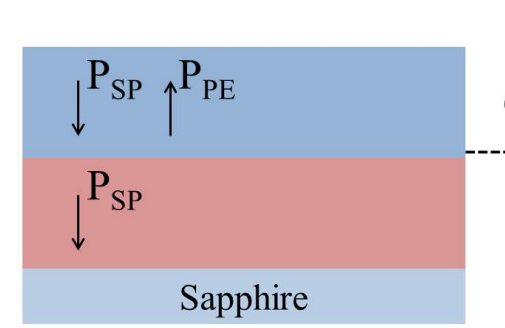

f) GaN Compressive Strain AlGaN Relaxed

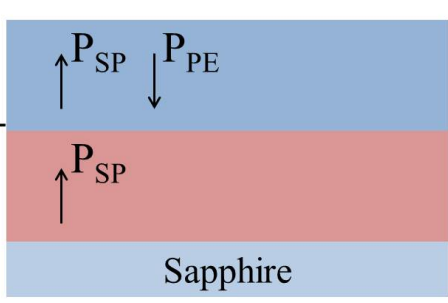

Figure 5.4: Polarization induced sheet charge densities, $\sigma$, and spontaneous and piezoelectric polarizations, $P_{S P}$ and $P_{P E}$ respectively, in the AlGaN/GaN heterojunction. Shown for both Ga-faced, [0001], and N-faced, [0001], polar GaN. Image modified from [92].

The spontaneous and piezoelectric polarizations for $\mathrm{Al}_{x} \mathrm{Ga}_{1-x} \mathrm{~N}$ are:

$$
\begin{gathered}
P_{S P}(x)=(-0.052 x-0.029) \mathrm{C} \cdot \mathrm{m}^{-2} \\
P_{P E}(x)=2 \frac{a(x)-a_{0}}{a_{0}}\left(e_{31}(x)-e_{33}(x) \frac{C_{13}(x)}{C_{33}(x)}\right) \quad \mathrm{C} \cdot \mathrm{m}^{-2}
\end{gathered}
$$

And the resulting polarization sheet charge density at the $\mathrm{Al}_{x} \mathrm{Ga}_{1-x} \mathrm{~N} / \mathrm{GaN}$ interface 
is:

$$
|\sigma(x)|=\left|P_{P E}\left(A l_{x} G a_{1-x} N\right)+P_{S P}\left(A l_{x} G a_{1-x} N\right)-P_{S P}(G a N)\right| \mathrm{C} \cdot \mathrm{m}^{-2}
$$

The polarization induced sheet charge carrier concentration is found to be:

$$
\eta_{s}(x)=\frac{+\sigma(x)}{e}-\left(\frac{\epsilon_{0} \epsilon(x)}{d e^{2}}\right)\left[e \Phi_{b}(x)+E_{F}(x)-\Delta E_{C}(x)\right] \mathrm{m}^{-2}
$$

Where:

$$
\begin{gathered}
E_{F}(x)=E_{0}(x)+\frac{\pi \hbar^{2}}{m^{*}(x)} \eta_{s}(x) \mathrm{eV} \\
E_{0}(x)=\left(\frac{9 \pi \hbar e^{2}}{\left.8 \epsilon_{0} \sqrt{8 m^{*}(x)} \frac{\eta_{s}(x)}{\epsilon(x)}\right)^{\frac{2}{3}} \mathrm{eV}}\right. \\
E_{g}(x)=x E_{C}=0.7\left[E_{g}(x)-E_{g}(0)\right] \mathrm{eV} \\
\epsilon(x)=-0.5 x+9.5 \\
e \Phi_{b}=(1.3 x+0.84) \mathrm{eV}
\end{gathered}
$$

The calculated polarization sheet charge density is shown in Figure 5.5. For $\mathrm{Al}_{x} \mathrm{Ga}_{1-x} \mathrm{~N}$ films of interest, where $0.2 \geq x \geq 0.4$, we see a calculated polarization sheet charge density of about 2 to $4 \times 10^{-6} \mathrm{C} / \mathrm{cm}^{2}$. This polarization will be compensated by the formation of a 2DEG at the AlGaN/GaN interface after growth, during cooling. 
The calculated polarization induced sheet charge concentration is shown in Figure 5.6. Typical $\mathrm{Al}_{x} \mathrm{Ga}_{1-x} \mathrm{~N}$ thicknesses for HEMT structures are on the order of $1030 \mathrm{~nm}$. The calculation predicts a sheet charge concentration in the low $1 \times 10^{13} \mathrm{~cm}^{-2}$ for $\mathrm{Al}_{x} \mathrm{Ga}_{1-x} \mathrm{~N}$ films where $x=0.25$ with a thickness of $2030 \mathrm{~nm}$.

For the samples grown, the AlN molar concentration was targeted to be $25 \%$ and the thickness was targeted to be $25 \mathrm{~nm}$ for the $\mathrm{Al}_{x} \mathrm{Ga}_{1-x} \mathrm{~N}$ layers. HRXRD and Hall effect measurements were used to determine the AlN molar concentration and the 2DEG sheet charge concentration. The hall effect results of the HEMT structures can be seen plotted on top of the data for $\mathrm{nGaN}$ in Figure 5.7. Here, it is obvious that a HEMT has bee grown because of the much larger mobilities at higher carrier concentrations when compared to typical nGaN. The experimental results were plotted on top of the calculated results and are shown in Figure 5.8. Here we see a good agreement between the simulated results and the experimental results. Some of the samples measured had

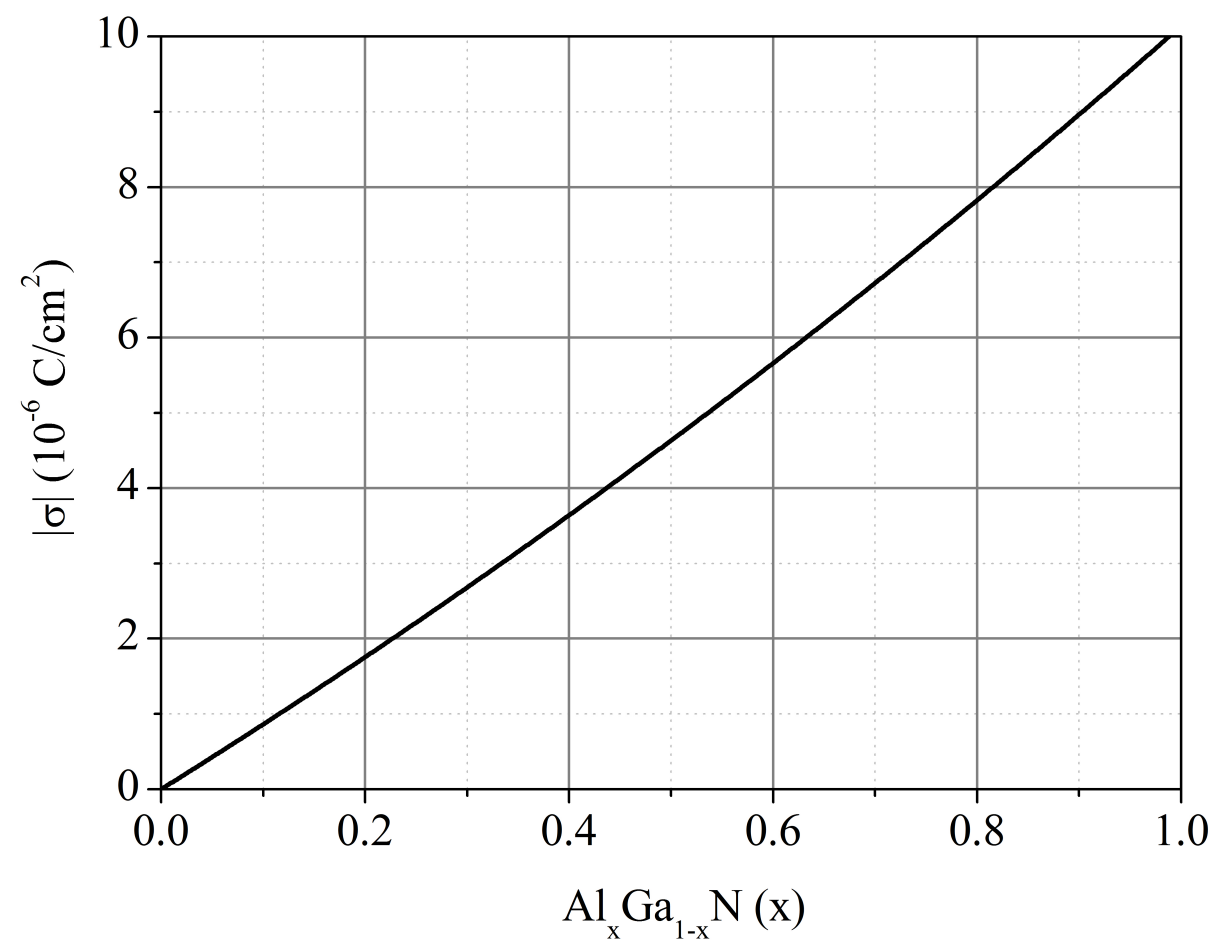

Figure 5.5: Calculated polarization sheet charge density as a function of the AlN molar fraction in the strained $A l_{x} G a_{1-x} N$ layer. 
slightly higher sheet charge concentration then calculated.

There are several possibilities for this discrepancy. There could be more un-

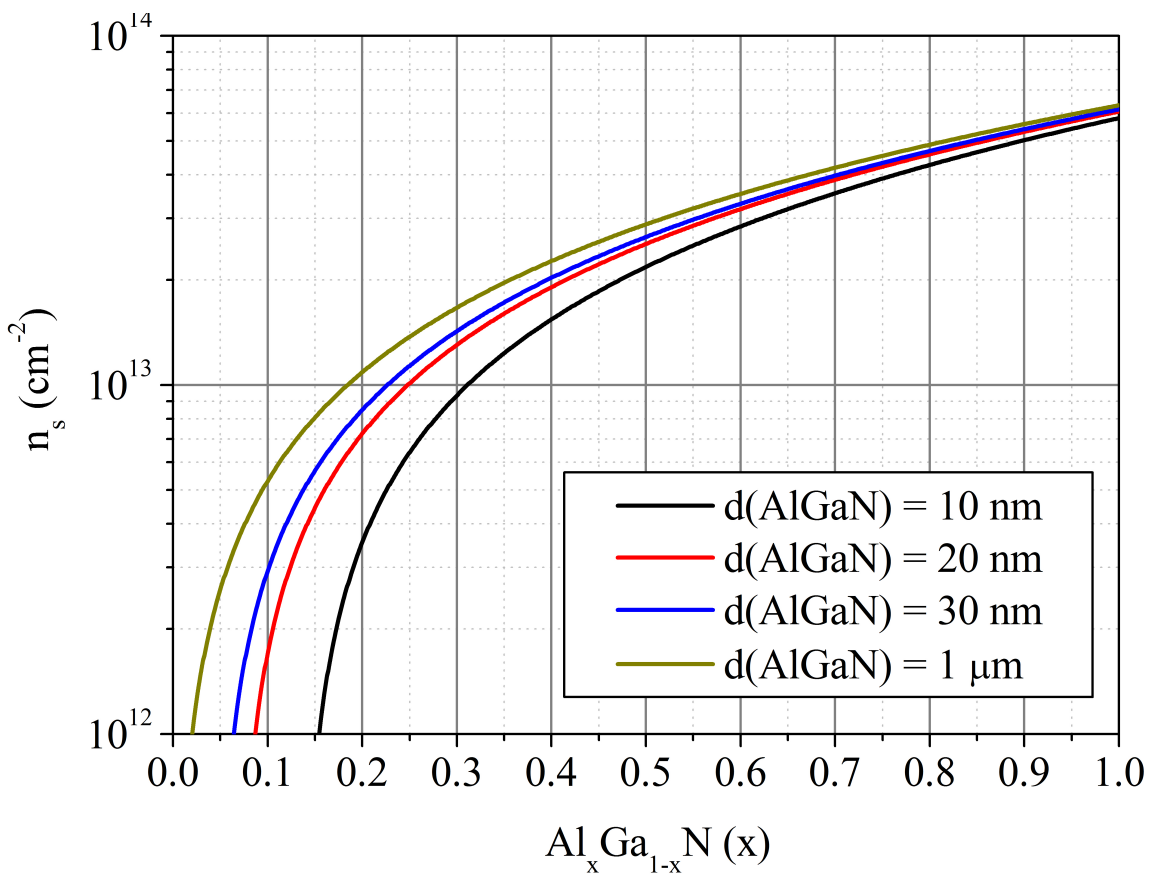

Figure 5.6: Calculated polarization induced sheet charge concentration as a function of the AlN molar fraction in the strained $A l_{x} G a_{1-x} N$ layer.

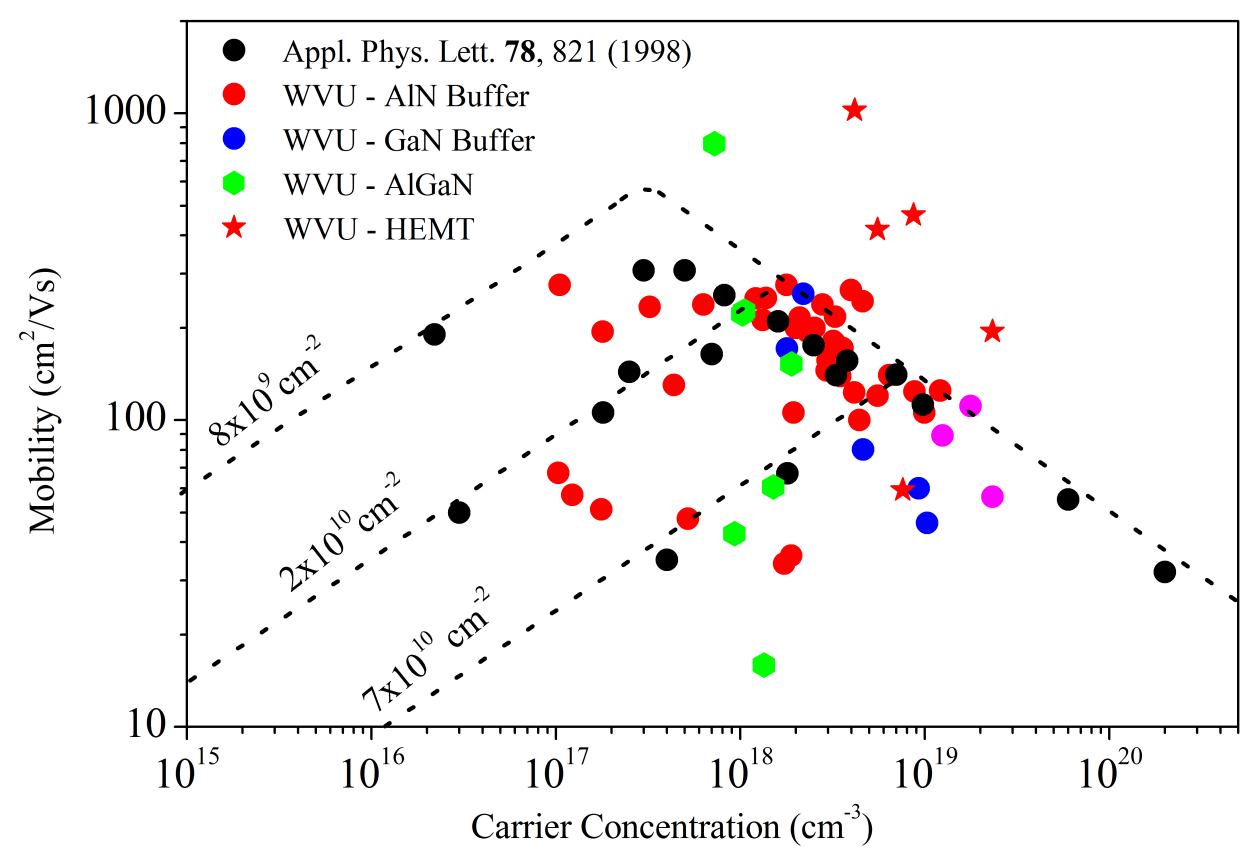

Figure 5.7: Carrier concentration vs. mobility for various n-type doped GaN and AlGaN films. 
intentional doping in some samples compared to other samples. In addition, samples for the Hall Effect measurement are scribed and cleaved by hand. Samples are cut into squares as best as possible, but sample geometries vary. This sample irregularity introduces some user error into the Hall Effect measurement. The calculation is also very simplistic, and does not take into account structural defects, unintentional doping and other unavoidable growth dependencies found in real films.

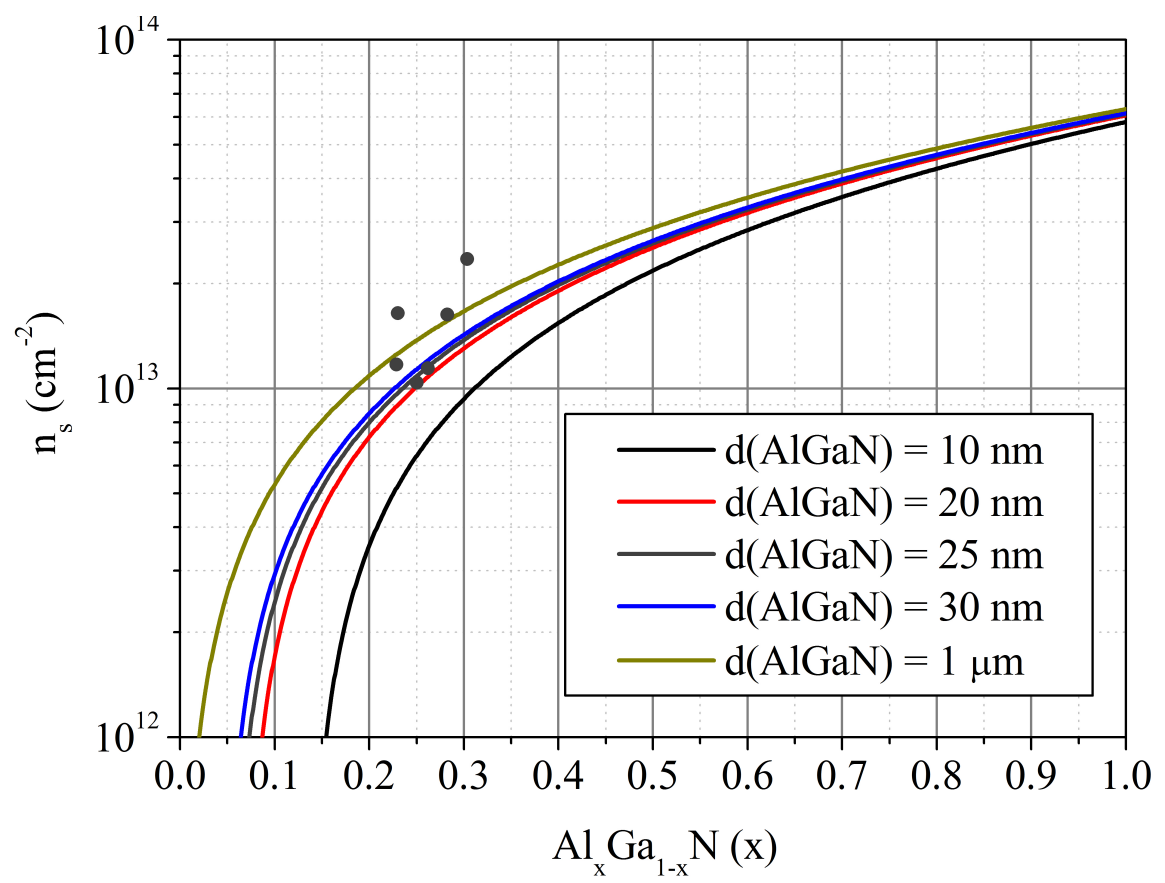

Figure 5.8: Experimental results for 2DEG sheet charge density found by HRXRD and Hall effect (shown as gray dots). 


\subsection{Experimental Results}

Figure 3.8 shows an optical image of a fabricated HEMT. In this image, the drain and source contacts are entirely on top of the AlGaN mesa. Other devices were fabricated with the Ohmic contacts overlapping the mesa edges. It was found that devices with Ohmic contacts on top of the mesa had an offset in the drain sweep. Currently this is attributed to the electron barrier in the band structure of the AlGaN/GaN heterojunction. When the contacts overlap the mesa edge, the drain sweeps pass through the origin as can be seen in Figure 5.9.

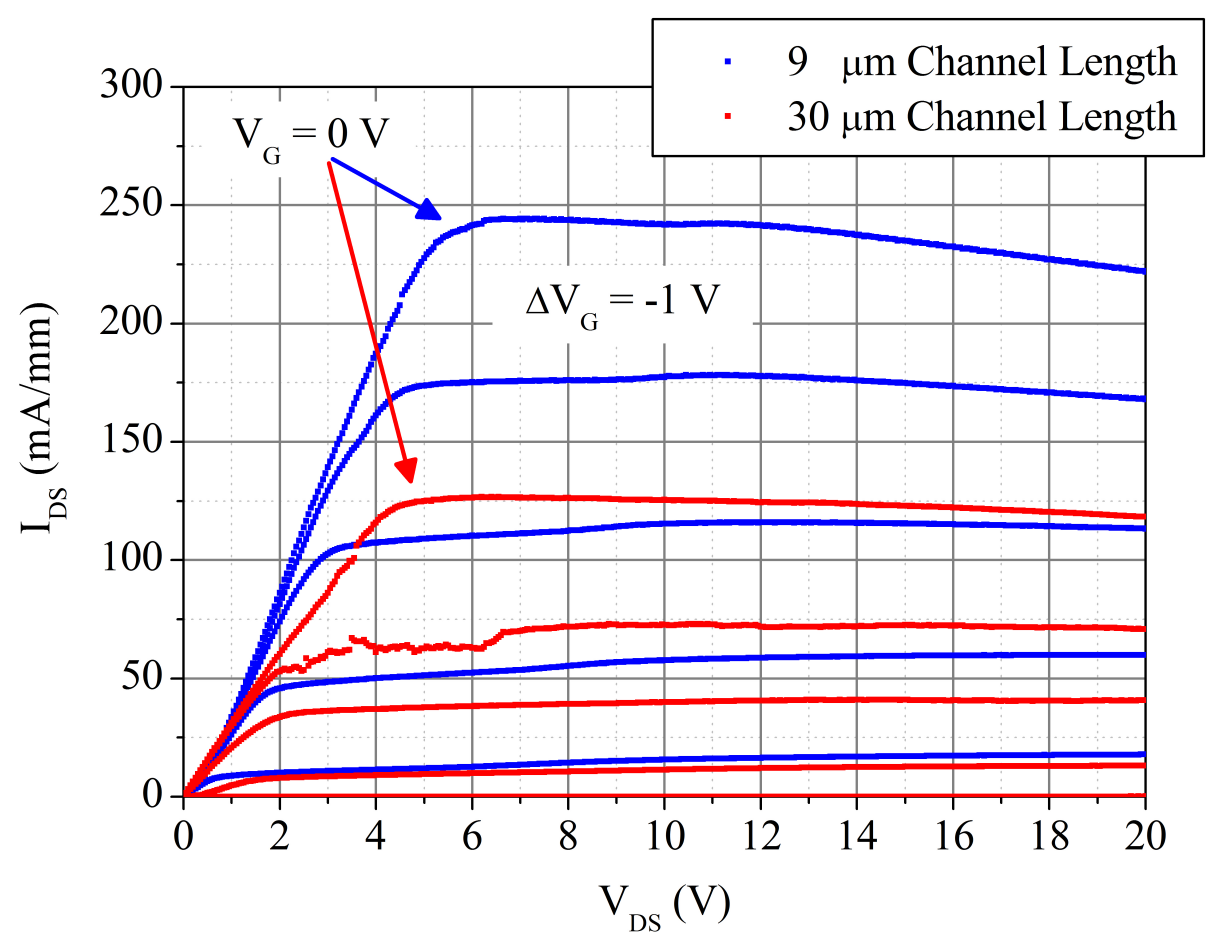

Figure 5.9: Drain sweep for HEMT devices with different channel lengths

Figure 5.9 shows the drain sweeps from two devices with 9 and $30 \mu \mathrm{m}$ channel lengths respectively (labeled in Figure 3.8). The $30 \mu \mathrm{m}$ and $9 \mu \mathrm{m}$ devices have a max drain current of about $250 \mathrm{~mA} / \mathrm{mm}$ and $125 \mathrm{~mA} / \mathrm{mm}$ at $0 \mathrm{~V}$ gate bias respectively.

Figure 5.10 shows the gate sweep of a $30 \mu \mathrm{m}$ channel length device. The current through the device is reduced approximately four orders of magnitude when it is in the off 
state. The turn on voltage is around $-3.5 \mathrm{~V}$ gate bias and the maximum transconductance is about $45 \mathrm{mS} / \mathrm{mm}$ at $-1 \mathrm{~V}$ gate bias.

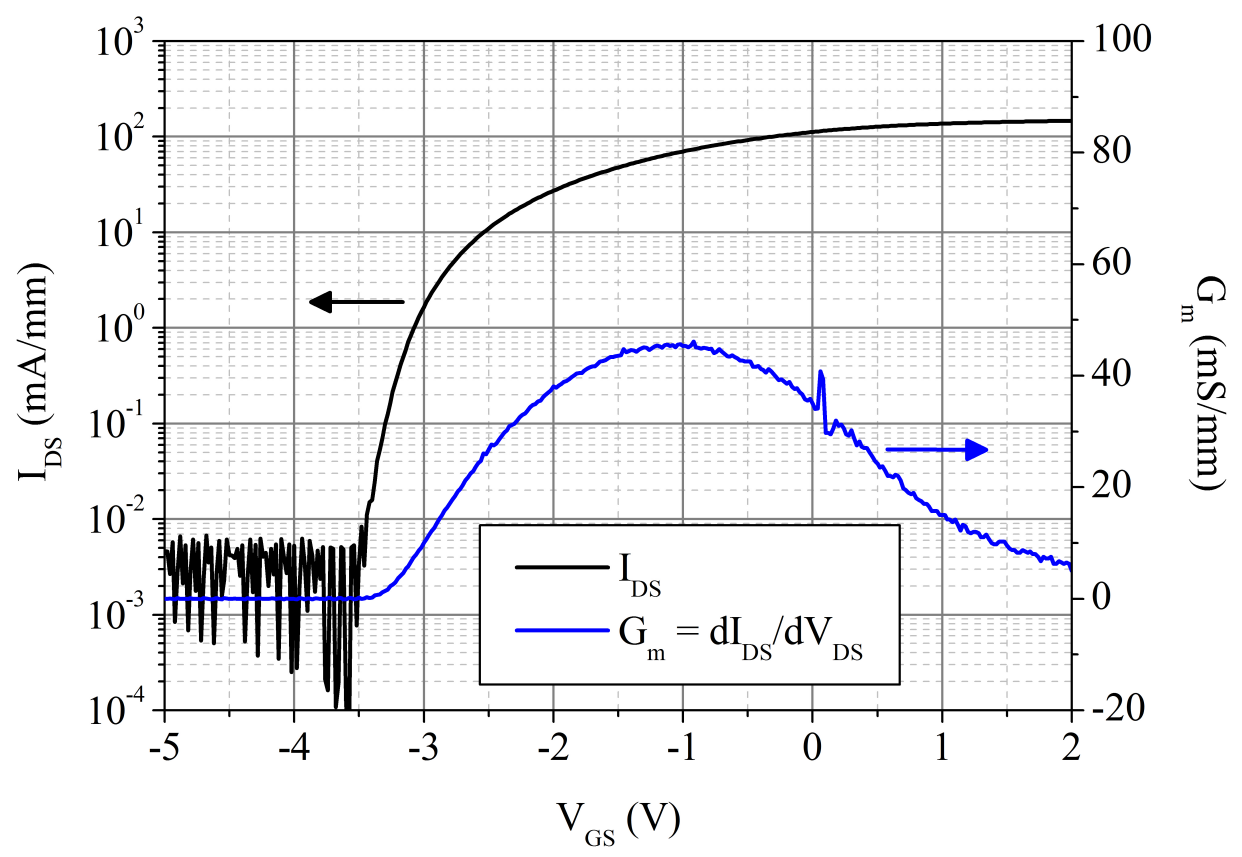

Figure 5.10: HEMT device gate sweep.

These results were typical of all the HEMT devices fabricated. While these device parameters are not as good as commercially available devices, which have drain currents on the order of $1 \mathrm{~A} / \mathrm{mm}$, they are very good considering the very simple epitaxial structure and fabrication process. 


\section{Chapter 6}

\section{Conclusions}

SAW wave devices were fabricated on GaN thin films on sapphire substrates with a thin AlN buffer layer. SAW frequency response was measured with a VNA and compared for different thicknesses and to the same SAW devices on $\mathrm{LiNbO}_{3}$ and thin film AlN. Rayleigh mode SAWs had insertion losses of about $40 \mathrm{~dB}$ for devices with $\lambda_{0}=32 \mu \mathrm{m}$ and GaN thickness of about $4 \mu \mathrm{m}$, compared to about $23 \mathrm{~dB}$ for the same devices on $\mathrm{LiNbO}_{3}$.

Non-Rayleigh mode SAWs were observed, and their propagation was dependent on crystal orientation. For SAW devices with $\lambda_{0}=8 \mu \mathrm{m}$ oriented along the $[11 \overline{2} 0]$ direction, 0th order Sezawa mode SAWs were observed at $1.435 \mathrm{GHz}$, which corresponds to an acoustic velocity of $11,480 \mathrm{~m} / \mathrm{s}$. This is slightly higher than the longitudinal acoustic velocity for sapphire. This mode is known as a pseudo-Sezawa SAW, and will only propagate in the GaN on sapphire systems with a small $k h_{\mathrm{GaN}}$ value. This work did not investigate Sezawa mode SAWs further, but future work could develop this mode of SAW for high frequency applications. 5th harmonic Sezawa mode SAWs would easily surpass $5 \mathrm{GHz}$ without sub-micron fabrication.

It has been shown that it is possible to excite the 5th, 7th, 9th and 11th harmonics SAW in GaN on sapphire. Bulk modes were shown to be suppressed with devices designed to operate at harmonic frequencies. In addition, the insertion loss at the center frequency for devices designed to operate at the fundamental, 5th, 7th, 9th and 11th harmonics were comparable. Devices, with a characteristic wavelength, $\lambda_{0}=20 \mu \mathrm{m}$, designed to operate at the fundamental, 5th, 7th, 9th and 11th harmonics had operating frequencies at 230, 962, 1338, 1720 and $2100 \mathrm{MHz}$ respectively. Operating frequencies 
in the GHz regime were realized with relatively large IDTs. Future work could involve developing more efficient harmonic devices. For example, 7 th harmonic devices with a metalization ratio, $\eta=0.35$ were superior to devices with $\eta=0.65$. The design parameters could be optimized for even better performing SAW devices at high harmonic frequencies.

The findings of this work also demonstrate that when $k h_{\mathrm{GaN}} \geq 10$, the effects of the sapphire substrate on the frequency response of a SAW device is negligible. Therefore, no alignment between the substrate and the devices is necessary, resulting in one less fabrication step. In addition, the relatively poor GaN quality at the GaN / sapphire interface also has no effect on the frequency response of devices where $k h_{\mathrm{GaN}} \geq 10$. High frequency operation also lends itself to thinner GaN films that maintain $k h_{\mathrm{GaN}} \geq 10$, improving device operation while lower fabrication costs.

GaN thin films were shown to resist oxidation and surface decomposition up to $600{ }^{\circ} \mathrm{C}$. SAW response on GaN did not degrade until thermal cycling at $750{ }^{\circ} \mathrm{C}$. After annealing at $850{ }^{\circ} \mathrm{C}, \mathrm{SAW}$ response was totally lost. It has been experimentally shown in this study that GaN is an excellent candidate for SAW devices operating up to 600 ${ }^{\circ} \mathrm{C}$. AlN films $1 \mu \mathrm{m}$ and thicker still need to be investigated. It is believed that SAW devices on AlN will have lower insertion loss than devices on GaN and will be able to operate up to $950{ }^{\circ} \mathrm{C}$ in atmosphere.

SAW devices on GaN thin films were tested in realistic exhaust gas environments. They were directly exposed to the exhaust gas of a 1992 Detroit Diesel Series 60 engine. Device performance was measured before and after exposure to exhaust gas through four important parameters; IL, SNR, OF and QF. In this work, the results indicate that GaN based SAW devices exhibit excellent chemical stability when exposed to diesel engine exhaust up to $465^{\circ} \mathrm{C}$. It has been demonstrated that 5 th and 7 th harmonic GaN based SAW devices are excellent candidates for gas sensors in high temperature, 
exhaust environments. More investigation is needed to identify what is occurring at the GaN surface, metallic contacts and the metal-semiconductor junction during exhaust testing. In the future scanning electron microscopy (SEM), atomic force microscopy (AFM) and X-ray photoelectric spectroscopy (XPS) will be utilized to characterize the material and supplement the electrical characterization.

It has been shown that electromagnetic feedthrough (EF) can be effectively reduced through different ground electrode geometries (GEG); however, there is no standard method for the reduction of $\mathrm{EF}$ and this typically results in a trial and error approach until a suitable configuration is acquired. Side-lobe rejection of SAW devices was effectively increased with a ground plane, improving the overall response. Different GEG were found to greater improve the device response of 5th and 7th harmonic devices by eliminated most of the EF. The SAW response of devices designed to operate beyond the 7th harmonic is severely degraded due to EF. In the future, different ground electrode configurations need to be explored to see if a reduction in the EF at higher frequencies can improve the response of the 9th and 11th harmonics.

HEMTs have been grown, fabricated and characterized. HEMTs fabricated in this work had drain currents of about $250 \mathrm{~mA} / \mathrm{mm}$ and $125 \mathrm{~mA} / \mathrm{mm}$ at $0 \mathrm{~V}$ gate bias for $30 \mu \mathrm{m}$ and $9 \mu \mathrm{m}$ channel length respectively. The $30 \mu \mathrm{m}$ channel length devices had a turn on voltage around $-3.5 \mathrm{~V}$ and maximum transconductance of approximately 45 $\mathrm{mS} / \mathrm{mm}$ at $-1 \mathrm{~V}$ gate bias. These device were sufficient to lay the groundwork for investigating different HEMT ICs. These first HEMTs were extremely simple structures with minimal fabrication. In the future, considerable optimization of the HEMT structure and fabrication process is possible, which would result in improved device operation. 

Appendices 



\title{
Appendix A
}

\section{Common Second Order SAW Effects and Design Solutions}

\author{
Source of Error Description of Error Design Solution
}

Surface IDTs can launch Bulk Acoustic Waves (BAW) that

IDT Launched travel through the substrate, Bulk Modes reflect off the bottom of the substrate and excite the receiving IDT.
Rough the bottom of the substrate to diffract and disperse BAW modes. Employ a Multi-strip Coupler (MSC) to laterally offset the launching and receiving IDTs.
Bidirectional IDTs launch SAWs

Rear Launched SAW from the rear of the IDT which can reflect back to the receiving IDT.
Deposit an acoustic absorbing material behind the lunching and receiving IDTs.
Multiple small reflections

Inter IDT

Reflections between each electrode in a single IDT can constructively interfere to create a noticeable effect.
Typically only noticeable with more than 20 electrodes in each IDT. Use a split electrode IDT design so inter IDT reflections cancel each other out. 


\section{Source of Error Description of Error Design Solution}

$\begin{array}{lll} & \text { Charge densities on individual } & \\ & \text { electrodes are not symmetric } & \text { Use of guard electrodes for IDTs } \\ \text { within IDTs containing few } & \text { with 20 or fewer electrodes. } \\ \text { End Effects } & \text { Make end effect negligible by } \\ & \begin{array}{l}\text { electrodes and this effect can } \\ \text { distort the frequency response of }\end{array} & \begin{array}{l}\text { designing IDTs with many } \\ \text { the SAW device. }\end{array}\end{array}$

Acoustic waves generated at

Triple Transit Response input reflect off output, then reflect off input and excite the output again at $3 t_{0}$, were $t_{0}$ is $v_{0} / d$.
For linear SAW device need to make the distance between IDTs large $>100 \lambda_{0}$, so that triple transit becomes negligible. Can also linearly offset IDTs through the use of a MSC.
Beam

Diffraction
If the aperture of the IDT is small, $<20 \lambda_{0}$, then beam diffraction is significant.
Make IDT aperture large > $20 \lambda_{0}$, so that beam diffraction becomes negligible.

Direct electromagnetic coupling occurs between IDTS through

Electromagnetic the air and through the

Feedthrough $(\mathrm{EF})$ substrate. EF through air is typically negligible. EF can become significant when a conductive substrate is used.

\section{Can use a higher resistivity} substrate, a non-conducting substrate, or deposit an insulating layer $\left(\mathrm{SiO}_{2}\right)$ between piezoelectric layer and substrate. 


\section{Appendix B}

\section{SAW Device Fabrication Procedures}

I. Sample Preparation
a) Wash for 5 minutes in acetone
b) Then, without letting acetone dry on surface, move to methanol and rinse for 5 minutes in the sonic agitator
c) Rinse in DI $\mathrm{H}_{2} \mathrm{O}$ for 10 minutes
d) Dehydration bake for 25 minutes at $120{ }^{\circ} \mathrm{C}$

II. SAW Device Image Reversal Photolithography
a) Spin AZ 5214-EIR for 40 seconds at $4000 \mathrm{rpm}$
b) Pre-bake for 60 seconds at $90{ }^{\circ} \mathrm{C}$
c) Mask exposure for $50 \mathrm{~mJ}$
d) Post-bake for 120 seconds at $120{ }^{\circ} \mathrm{C}$ (Most Crucial Step)
e) Flood exposure for $2000 \mathrm{~mJ}$
f) Develop in a solution of 250:75 of AZ 400K:H2O for $\approx 25$ seconds
g) Verify pattern under optical microscope
h) Verify pattern with $\alpha$-step (depth should be $1.4 \mu \mathrm{m}$ )

III. Ohmic Contact Deposition in E-Beam Evaporator
a) Deposit 2/100 nm of $\mathrm{Ti} / \mathrm{Al}$ or 2/80 $\mathrm{nm}$ of $\mathrm{Ni} / \mathrm{Pt}$, depending on application
b) Liftoff in acetone (Do not put in Sonic Agitator!!)
c) Clean in Acetone / Methanol / DI $\mathrm{H}_{2} \mathrm{O} /$ Dehydration Bake for 25 minutes at $120{ }^{\circ} \mathrm{C}$

IV. SAW Device Annealing in RTA

a) Anneal in $\mathrm{N}_{2}$ for 300 seconds at $300{ }^{\circ} \mathrm{C}$ 



\section{Appendix C}

\section{HEMT Fabrication Procedures}

I. Sample Preparation
a) Wash for 5 minutes in acetone, then, without letting acetone dry on surface, move to methanol and rinse for 5 minutes in the sonic agitator
b) Oxide etch in BOE for 3 minutes
c) Rinse in DI $\mathrm{H}_{2} \mathrm{O}$ for 2 minutes
d) Surface clean in a $1: 1$ solution of $\mathrm{HCl}: \mathrm{H}_{2} \mathrm{O}$ for 1.5 minutes
e) Rinse in DI $\mathrm{H}_{2} \mathrm{O}$ for 2 minutes
f) Dehydration bake for 25 minutes at $120{ }^{\circ} \mathrm{C}$

II. Mesa Etch Positive Photolithography
a) Spin AZ 5214-EIR for 40 seconds at $4000 \mathrm{rpm}$
b) Pre-Bake for 60 seconds at $90{ }^{\circ} \mathrm{C}$
c) Mask Exposure for $100 \mathrm{~mJ}$
d) Develop in undiluted $300 \mathrm{MIF}$
e) Hard bake for 20 minutes at $120^{\circ} \mathrm{C}$
f) Verify pattern under optical microscope
g) Verify pattern with $\alpha$-step (height should be $1.4 \mu \mathrm{m}$ )

III. Mesa Etch in ICP
a) $\mathrm{O}_{2}$ clean for 10 minutes
b) Chamber condition for 10 minutes
c) Etch for at least $50 \mathrm{~nm}$
i. Rate $3.5 \mathrm{~nm} / \mathrm{s}(9 / 28 / 12)$
ii. Need to verify etch rate 
iii. Need to optimize process for $\mathrm{Al}_{x} \mathrm{Ga}_{1-x} \mathrm{~N}$

d) Remove etch mask in acetone bath

e) Verify mesa height with $\alpha$-step

f) Clean in acetone / methanol / DI $\mathrm{H}_{2} \mathrm{O} /$ dehydration bake for 25 minutes at $120{ }^{\circ} \mathrm{C}$

IV. Ohmic Contact Image Reversal Photolithography

a) Spin AZ 5214-EIR for 40 seconds at $4000 \mathrm{rpm}$

b) Pre-bake for 60 seconds at $90{ }^{\circ} \mathrm{C}$

c) Mask exposure for $50 \mathrm{~mJ}$

d) Post-bake for 120 seconds at $120{ }^{\circ} \mathrm{C}$ (Most Crucial Step)

e) Flood exposure for $2000 \mathrm{~mJ}$

f) Develop in a solution of 250:75 of $\mathrm{AZ} 400 \mathrm{~K}: \mathrm{H} 2 \mathrm{O}$ for $\approx 25$ seconds

g) Verify pattern under optical microscope

h) Verify pattern with $\alpha$-step (depth should be $1.4 \mu \mathrm{m}$ )

V. Ohmic Contact Evaporation in E-Beam Evaporator

\begin{tabular}{cccccc}
\hline Metal & $\begin{array}{c}\text { Thickness } \\
(\mathrm{nm})\end{array}$ & $\begin{array}{c}\text { Dep.Rate } \\
(\AA / \mathrm{s})\end{array}$ & TF & Density & $Z$-Ratio \\
\hline $\mathrm{Ti}$ & 30 & 0.1 & 46.5 & 4.5 & 0.628 \\
$\mathrm{Al}$ & 225 & 3 & 50 & 2.7 & 1.080 \\
$N i$ & 80 & 2 & 55 & 8.91 & 0.331 \\
$A u$ & 40 & 3 & 34 & 19.3 & 0.381 \\
\hline
\end{tabular}

b) Liftoff in acetone (Do not put in Sonic Agitator!!)

c) Clean in Acetone / Methanol / DI $\mathrm{H}_{2} \mathrm{O} /$ Dehydration Bake for 25 minutes at $120^{\circ} \mathrm{C}$

VI. Ohmic Contact Annealing in RTA

a) Anneal in $\mathrm{N}_{2}$ for 30 seconds at $850{ }^{\circ} \mathrm{C}$

VII. Schottky Contact Image Reversal Photolithography

a) Spin AZ 5214-EIR for 40 seconds at $4000 \mathrm{rpm}$ 
b) Pre-bake for 60 seconds at $90{ }^{\circ} \mathrm{C}$

c) Mask exposure for $50 \mathrm{~mJ}$

d) Post-bake for 120 seconds at $120{ }^{\circ} \mathrm{C}$ (Most Crucial Step)

e) Flood exposure for $2000 \mathrm{~mJ}$

f) Develop in a solution of 250:75 of AZ 400K:H2O for $\approx 25$ seconds

g) Verify pattern under optical microscope

h) Verify pattern with $\alpha$-step (depth should be $1.4 \mu \mathrm{m}$ )

VIII. Schottky Contact Evaporation in E-Beam Evaporator

Metal Thickness Dep.Rate TF Density Z-Ratio

a) \begin{tabular}{rccccc}
\multicolumn{7}{c}{$(\mathrm{nm})$} & $(\AA / s)$ \\
\hline$N i$ & 30 & 2 & 55 & 8.91 & 0.331 \\
$A u$ & 40 & 3 & 34 & 19.3 & 0.381 \\
\hline
\end{tabular}

b) Liftoff in acetone (Do not put in Sonic Agitator!!)

c) Clean in acetone / methanol / DI $\mathrm{H}_{2} \mathrm{O} /$ dehydration bake for 25 minutes at $120{ }^{\circ} \mathrm{C}$

IX. Bonding Pad Image Reversal Photolithography

a) Spin AZ 5214-EIR for 40 seconds at $4000 \mathrm{rpm}$

b) Pre-bake for 60 seconds at $90{ }^{\circ} \mathrm{C}$

c) Mask exposure for $50 \mathrm{~mJ}$

d) Post-bake for 120 seconds at $120{ }^{\circ} \mathrm{C}$ (Most Crucial Step)

e) Flood exposure for $2000 \mathrm{~mJ}$

f) Develop in a solution of 250:75 of AZ 400K:H2O for $\approx 25$ seconds

g) Verify pattern under Optical Microscope

h) Verify pattern with $\alpha$-step (Depth should be $1.4 \mu \mathrm{m}$ )

X. Bonding Pad Deposition in Sputtering Station

a) Sputter $600 \mathrm{~nm}$ of $\mathrm{Au}$

b) Liftoff in Acetone (Do not put in Sonic Agitator!!) 
c) Clean in Acetone / Methanol / DI $\mathrm{H}_{2} \mathrm{O} /$ Dehydration Bake for 25 min at $120{ }^{\circ} \mathrm{C}$

XI. Anneal Sample in RTA
a) Anneal in $\mathrm{N}_{2}$ for 5 minutes at $300{ }^{\circ} \mathrm{C}$

XII. Clean Sample
a) Clean with acetone in sonic agitator for 5 minutes
b) Clean with methanol 5 minutes
c) Rinse with DI $\mathrm{H}_{2} \mathrm{O}$
d) Dry with compressed $\mathrm{N}_{2}$ 


\section{Appendix D}

\section{VNA Calibration and Operation}

Systematic errors can be greatly reduced through measurement calibration, so that their effects can be considered negligible. To perform a measurement calibration on the Agilent E8362B VNA a custom calibration kit (Cal Kit) must first be created. To do this, the following procedures are outlined:

1. Click on the Calibration menu button

2. Click on Advanced Modify Cal Kit

3. Click on Insert New

4. Give your Cal Kit a name

5. Use the follow parameters for the Cascade Microtech ACP40-GSG-150 probes

a. C-open (On Substrate) $(\mathrm{fF})=3.5$

b. L-short $(\mathrm{pH})=4.8$

c. Load Impedance $(\Omega)=50$

d. Thru Delay $(\mathrm{ps})=1.0$

6. Select the SOLT calibration type

\section{Click Finish}

To perform the measurement calibration, place the Cascade Microtech Impedance Standard Substrate (ISS) on the probe station chuck. Hook up probes and cables to the VNA and click on the Calibration button. Click on Calibration Wizard, select SmartCal, click Next, and follow the on screen instructions. To ensure a proper calibration, a certain amount of overtravel is necessary when contacting the probes to the ISS. Once you have finished making the measurement calibration, be sure automatic correction is turned on and the correct calibration is selected before making measurements. 
Random errors can be reduced by performing a time average during data collection. Random errors will always add up to zero over a long enough period of time. If they dont, they are not truly random. By averaging results over a period of time, the net effect of random errors can be reduced to a negligible amount. The VNA easily does this and all that is needed is to select Average under the Channel button and set the desired number of iterations before averaging will finish.

The following steps are followed whenever collecting data with the VNA. The only step that changes with different measurement types is step $4 \mathrm{~b}$. In this step, it is simply necessary to select the appropriate measurement type.

1. Preset the analyzer (Set the frequency span).

2. Connect the device under test (DUT) to the VNA.

3. Select the $\mathrm{S}_{21}$ measurement.

4. Set the following settings:

a. Number of measurement points. (Typically 16001)

b. Measurement format. (Phase, $-\mathrm{S}_{21}-$, Group Delay, etc.)

c. Scale.

5. Remove the DUT and perform a measurement calibration.

6. Reconnect the DUT.

7. Scale the displayed measurement.

8. Turn on the VNAs smoothing function and set the aperture.

9. Set markers and save data. 


\section{Bibliography}

[1] L. Rayleigh, "On Waves Propagated along the Plane Surface of an Elastic Solid," Proc. London Math. Soc., vol. s1-17, no. 1, pp. 4-11, 1885. 5

[2] J. L. Rose. Cambridge University Press, 1st ed., Sep 2004. 5

[3] J. D. N. Cheeke, Fundamentals and Applications of Ultrasonic Waves. CRC Press, 2nd ed., Jun 2012. 5

[4] T. M. Gronewold, "Surface acoustic wave sensors in the bioanalytical field: Recent trends and challenges," Anal. Chim. Acta, vol. 603, no. 2, pp. 119-128, 2007. 5, 6

[5] D. S. B. Jr., R. M. White, S. J. Martin, E. T. Zellers, G. C. Frye, and H. Wohltjen. Academic Press, 1st ed., Oct 1996. 5, 6, 28, 45

[6] R. M. White and F. W. Voltmer, "Direct piezoelectric coupling to surface elastic waves," Appl. Phys. Lett., vol. 7, pp. 314-316, Dec 1965. 6

[7] F. Hickernell, "Surface acoustic wave devices: a rewarding past, a significant present, and a promising future," in 12th International Conference on Microwaves and Radar, MIKON '98., vol. 4, pp. 159-168, 1998. 6, 8

[8] C. Campbell, "Applications of surface acoustic and shallow bulk acoustic wave devices," Proc. IEEE, vol. 77, no. 10, pp. 1453-1484, 1989. 7

[9] E. E. Boudouti, B. Djafari-Rouhani, A. Akjouj, and L. Dobrzynski, "Acoustic waves in solid and fluid layered materials," Sur. Sci. Rep., vol. 64, no. 11, pp. 471-594, 2009. 6

[10] W. A. Groves and E. T. Zellers, "Analysis of solvent vapors in breath and ambient air with a surface acoustic wave sensor array," The Annals of Occupational Hygiene, vol. 45, no. 8, pp. 609-623, 2001. 6

[11] K.-S. Chang, C.-K. Chang, and C.-Y. Chen, "A surface acoustic wave sensor modified from a wireless transmitter for the monitoring of the growth of bacteria," Sens. Act. $B$, vol. 125, no. 1, pp. 207-213, 2007. 6

[12] F. D. Pietrantonio, M. Benetti, D. Cannatà, E. Verona, A. Palla-Papavlu, V. Dinca, M. Dinescu, T. Mattle, and T. Lippert, "Volatile toxic compound detection by surface acoustic wave sensor array coated with chemoselective polymers deposited by laser induced forward transfer: Application to sarin," Sens. Act. B, vol. 174, no. 0 , pp. $158-167,2012.6$ 
[13] O. Onen and R. Guldiken, "Investigation of Guided Surface Acoustic Wave Sensors by Analytical Modeling and Perturbation Analysis," Sens. Act. A, no. 0, pp.--, 2013. 6

[14] G. N. Ferreira, A.-C. da Silva, and B. Tomé, "Acoustic wave biosensors: physical models and biological applications of quartz crystal microbalance," Trends in Biotechnology, vol. 27, no. 12, pp. 689-697, 2009. 6

[15] Y. W. Kim, S. E. Sardari, M. T. Meyer, A. A. Iliadis, H. C. Wu, W. E. Bentley, and R. Ghodssi, "An $\{$ ALD $\}$ aluminum oxide passivated Surface Acoustic Wave sensor for early biofilm detection," Sens. Act. B, vol. 163, no. 1, pp. 136-145, 2012. 6

[16] F. Yam and Z. Hassan, "Ingan: An overview of the growth kinetics, physical properties and emission mechanisms," Superlattices and Microstructures, vol. 43, no. 1, pp. $1-23,2008.8$

[17] Z. Qin, Z. Chen, Y. Tong, S. Lu, and G. Zhang, "Estimation of inn phase inclusion in ingan films grown by movpe," Applied Physics A, vol. 74, no. 5, pp. 655-658, 2002. 8

[18] D. of Energy, "Solid state lighting research and development: Multi year program plan," March 2012. 8, 9

[19] "Index," in Nitride Semiconductor Light-Emitting Diodes (LEDs) (J. Huang, H.-C. Kuo, and S.-C. Shen, eds.), pp. 607 - 624, Woodhead Publishing, 2014. 9

[20] O. Ambacher, "Growth and applications of group iii-nitrides," Journal of Physics D: Applied Physics, vol. 31, no. 20, p. 2653, 1998. 9, 10

[21] U. K. Mishra, P. Parikh, and Y.-F. Wu, "Algan/gan hemts-an overview of device operation and applications," Proceedings of the IEEE, vol. 90, pp. 1022-1031, Jun 2002. 10

[22] V. Cimalla, J. Pezoldt, and O. Ambacher, "Group iii nitride and sic based mems and nems: materials properties, technology and applications," Journal of Physics D: Applied Physics, vol. 40, no. 20, p. 6386, 2007. 10

[23] V. Kumbham, "Synthesis and Characterization of electrodes for III-Nitride Resonant Cavity Light Emitting Diode Applications," PhD. Thesis, West Virginia University, 2012. 20

[24] L. Turlapati, "Developing UV Photodetector and Ohmic Contact Techniques on GaN," PhD. Thesis, West Virginia University, 2004. 20

[25] A. Technologies, Agilent Network Analyzer Basics, vol. 1. Agilent, 2004. 22, 23 
[26] J. Justice, L. E. Rodak, K. Lee, L. A. Hornak, and D. Korakakis, "Harmonic Excitation of Surface Acoustic Waves on Gallium Nitride Thin Films for Biological and Chemical Sensor Applications," Proc. Mater. Res. Soc., vol. 1415, 1 2012. 26, $28,34,51$

[27] C. K. Campbell. Academic Press, 1st ed., Apr 1989. 28

[28] R. McGill, R. Chung, D. Chrisey, P. Dorsey, P. Matthews, A. Pique, T. Mlsna, and J. Stepnowski, "Performance optimization of surface acoustic wave chemical sensors," IEEE Trans. on Ultrason., Ferroelec., and Freq. Cont., vol. 45, no. 5, pp. $1370-1380,1998.28$

[29] A. Afzal, N. Iqbal, A. Mujahid, and R. Schirhagl, "Advanced vapor recognition materials for selective and fast responsive surface acoustic wave sensors: A review," Anal. Chim. Acta, vol. 787, no. 0, pp. 36-49, 2013. 29

[30] M. Assouar, M. E. Hakiki, O. Elmazria, P. Alnot, and C. Tiusan, "Synthesis and microstructural characterisation of reactive $\{\mathrm{RF}\}$ magnetron sputtering AlN films for surface acoustic wave filters," Diamond and Related Materials, vol. 13, no. 48, pp. 1111-1115, 2004. 14th European Conference on Diamond, Diamond-Like Materials, Carbon Nanotubes, Nitrides and Silicon Carbide. 29

[31] M. Assouar, O. Elmazria, L. L. Brizoual, and P. Alnot, "Reactive $\{$ DC $\}$ magnetron sputtering of aluminum nitride films for surface acoustic wave devices," Diamond and Related Materials, vol. 11, no. 3-6, pp. 413-417, 2002. 12th European Conference on Diamond, Diamond- Like Materials, Carbon Nanotubes, Nitrides \&amp; Silicon Carbide. 29

[32] V. Mortet, O. Elmazria, M. Nesladek, J. D'Haen, G. Vanhoyland, M. Elhakiki, A. Tajani, E. Bustarret, E. Gheeraert, M. D'Olieslaeger, and P. Alnot, "Study of aluminium nitride/freestanding diamond surface acoustic waves filters," Diamond and Related Materials, vol. 12, no. 3-7, pp. 723-727, 2003. 13th European Conference on Diamond, Diamond-Like Materials, Carbon Nanotubes, Nitrides and Silicon Carbide. 29

[33] K. Kao, C. Cheng, Y. Chen, and C. Chen, "The dispersion properties of surface acoustic wave devices on AlN/LiNbO3 film/substrate structure," Appl. Sur. Sci., vol. 230, no. 1-4, pp. 334-339, 2004. 29

[34] D.-T. Phan and G.-S. Chung, "The effect of geometry and post-annealing on surface acoustic wave characteristics of AlN thin films prepared by magnetron sputtering," Appl. Sur. Sci., vol. 257, no. 20, pp. 8696-8701, 2011. 29

[35] F. Bénédic, M. Assouar, F. Mohasseb, O. Elmazria, P. Alnot, and A. Gicquel, "Surface acoustic wave devices based on nanocrystalline diamond and aluminium 
nitride," Diamond and Related Materials, vol. 13, no. 2, pp. 347-353, 2004. Carbon Materials for Active Electronics. Proceedings of Symposium L, E-MRS Spring Meeting 2003. 29

[36] M. Benetti, D. Cannatà, F. D. Pietrantonio, E. Verona, A. Generosi, B. Paci, and V. R. Albertini, "Growth and characterization of piezoelectric AlN thin films for diamond-based surface acoustic wave devices," Thin Solid Films, vol. 497, no. 1-2, pp. 304-308, 2006. 29

[37] J.-H. Song, J.-L. Huang, T. Omori, J. C. Sung, S. Wu, H.-H. Lu, and D.-F. Lii, "Growth of highly c-axis oriented $(\mathrm{B}, \mathrm{Al}) \mathrm{N}$ film on diamond for high frequency surface acoustic wave devices," Thin Solid Films, vol. 520, no. 6, pp. 2247-2250, 2012. 29

[38] P. Chalker, T. Joyce, C. Johnston, J. Crossley, J. Huddlestone, M. Whitfield, and R. Jackman, "Fabrication of aluminium nitride/diamond and gallium nitride/diamond $\{$ SAW $\}$ devices," Diamond and Related Materials, vol. 8, no. 2-5, pp. 309-313, 1999. 29

[39] T. Lalinský, L. Rufer, G. Vanko, S. Mir, v. Haščík, v. Mozolová, A. Vincze, and F. Uherek, "AlGaN/GaN heterostructure-based surface acoustic wave-structures for chemical sensors," Appl. Sur. Sci., vol. 255, no. 3, pp. 712-714, 2008. 11th International Conference on the Formation of Semiconductor Interfaces (ICFSI-11). 29

[40] F. Semond, D. Schenck, M. Jibard, S. Camou, T. Pastureaud, A. Soufyane, and S. Ballandras, "Epitaxy of $\{$ AIN $\}$ and GaN thin films on silicon or sapphire for the development of high frequency saw devices," Annales de Chimie Science des Matériaux, vol. 26, no. 1, pp. 177-182, 2001. 29

[41] I. Rýger, G. Vanko, T. Lalinský, M. Vallo, M. Tomáška, and A. Ritomský, "AlGaN/GaN Based SAW-HEMT Devices for Chemical Gas Sensors Operating in \{GHz\} Range," Proc. Eng., vol. 25, no. 0, pp. 1101-1104, 2011. EurosensorsXXV. 29,33

[42] J. Justice, K. Lee, and D. Korakakis, "Harmonic surface acoustic waves on gallium nitride thin films," IEEE Trans. on Ultrason., Ferroelec., and Freq. Cont., vol. 59, no. 8, pp. 1806-1811, 2012. 32, 36, 37, 39

[43] M. Levy, H. Bass, and R. Stern, eds. Academic Press, 1st ed., Oct 2001. 32

[44] K. H. Choi, H. Joon, S. J. Chung, J. Y. Kim, T. K. Lee, and Y. J. Kim, "Experimental and theoretical characterization of the surface acoustic wave propagation properties of GaN epitaxial layers on c-plane sapphire," J. Mater. Res., vol. 18, pp. 1157-1161, 5 2003. Ref C for GaN Dispersion Figure. 33, 38 
[45] J. Rodríguez-Madrid, G. Iriarte, O. Williams, and F. Calle, "High precision pressure sensors based on $\{\mathrm{SAW}\}$ devices in the $\{\mathrm{GHz}\}$ range," Sens. Act. A, vol. 189 , no. 0 , pp. 364-369, 2013. 33

[46] J. Rodríguez-Madrid, G. Iriarte, D. Araujo, M. Villar, O. Williams, W. MüllerSebert, and F. Calle, "Optimization of AlN thin layers on diamond substrates for high frequency SAW resonators," Mater. Lett., vol. 66, no. 1, pp. 339-342, 2012. 33

[47] C.-L. Wei, Y.-C. Chen, C.-C. Cheng, K.-S. Kao, D.-L. Cheng, and P.-S. Cheng, "Highly sensitive ultraviolet detector using a ZnO/Si layered \{SAW oscillator," Thin Solid Films, vol. 518, no. 11, pp. 3059-3062, 2010. Transparent Oxides for Electronics and Optiocs. 33

[48] R. Ro, R. Lee, Z.-X. Lin, C.-C. Sung, Y.-F. Chiang, and S. Wu, "Surface acoustic wave characteristics of a (100) $\mathrm{ZnO} /(100)$ AlN/diamond structure," Thin Solid Films, vol. 529, no. 0, pp. 470-474, 2013. TACT2011 International Thin Films Conference. 33

[49] L. L. Brizoual, O. Elmazria, F. Sarry, M. E. Hakiki, A. Talbi, and P. Alnot, "High frequency SAW devices based on third harmonic generation," Ultrasonics, vol. 45, no. 1-4, pp. 100-103, 2006. 33

[50] P. Naraine, C. K. Campbell, and Y. Ye, "A SAW Step-Type Delay Line for Efficient High Order Harmonic Mode Excitation," Proc. IEEE Ultrason. Symp., vol. 10, pp. 322-325, 1980. 33

[51] C. Campbell and P. Edmonson, "An empirical method for obtaining the harmonic response coefficients of a SAW interdigital transducer," in Proc. IEEE Ultrason. Symp., vol. 1, pp. 283-287 vol.1, 2002. 34, 37

[52] S.-H. Lee, H.-H. Jeong, S.-B. Bae, H.-C. Choi, J.-H. Lee, and Y. Lee, "Epitaxially grown GaN thin-film SAW filter with high velocity and low insertion loss," IEEE Trans. Electron Devices, vol. 48, no. 3, pp. 524-529, 2001. Ref A for GaN Dispersion Figure. 38

[53] S. Petroni, G. Tripoli, C. Combi, B. Vigna, M. D. Vittorio, M. Todaro, G. Epifani, R. Cingolani, and A. Passaseo, "GaN-based surface acoustic wave filters for wireless communications," Superlattices and Microstructures, vol. 36, no. 4-6, pp. 825-831, 2004. Ref B for GaN Dispersion Figure. 38

[54] J. Pedrós, F. Calle, J. Grajal, R. J. Riobóo, C. Prieto, J. Pau, J. Pereiro, M. Hermann, M. Eickhoff, and Z. Bougrioua, "Anisotropic propagation of surface acoustic waves on nitride layers," Superlattices and Microstructures, vol. 36, no. 4-6, pp. 815823, 2004. 38, 39

[55] S. Sakharov, D. Roshchupkin, E. Emelin, D. Irzhak, O. Buzanov, and A. Zabelin, "X-ray diffraction investigation of high-temperature SAW-sensor based on LGS crystal," Proc. Eng., vol. 25, no. 0, pp. 1020-1023, 2011. EurosensorsXXV. 40 
[56] G. Tortissier, L. Blanc, A. Tetelin, J.-L. Lachaud, M. Benoit, V. Conédéra, C. Dejous, and D. Rebière, "Langasite based surface acoustic wave sensors for high temperature chemical detection in harsh environment: Design of the transducers and packaging," Sens. Act. B, vol. 156, no. 2, pp. 510-516, 2011. 40

[57] J. Thiele and M. P. da Cunha, "High temperature LGS SAW gas sensor," Sens. Act. B, vol. 113, no. 2, pp. 816-822, 2006. Special Issue - In honour of Professor Karl Cammann. 40

[58] E. Ansorge, S. Schimpf, S. Hirsch, J. Sauerwald, H. Fritze, and B. Schmidt, "Evaluation of langasite (La3Ga5SiO14) as a material for high temperature microsystems," Sens. Act. A, vol. 130-131, no. 0, pp. 393-396, 2006. 40

[59] G. Tortissier, L. Blanc, A. Tetelin, J.-L. Lachaud, M. Benoit, V. Conédéra, C. Dejous, and D. Rebière, "Langasite Based Surface Acoustic Wave Sensors for High Temperature Chemical Detection in Harsh Environment," Proc. Chem., vol. 1, no. 1, pp. 963-966, 2009. Proceedings of the Eurosensors $\{$ XXIII $\}$ conference;. 40

[60] G. Bruckner, T. Aubert, J. Bardong, D. Eisele, R. Fachberger, E. Mayer, and S. Salzmann, "Investigations of several langasite cuts by high temperature in situ characterizations of surface acoustic waves delay lines," Proc. Eng., vol. 25, no. 0, pp. 317-320, 2011. EurosensorsXXV. 40

[61] M. E. Hakiki, O. Elmazria, F. Bénédic, P. Nicolay, D. Monéger, and R. Azouani, "Diamond film on Langasite substrate for surface acoustic wave devices operating in high frequency and high temperature," Diamond and Related Materials, vol. 16, no. 4-7, pp. 966-969, 2007. Proceedings of Diamond 2006, the 17th European Conference on Diamond, Diamond-Like Materials, Carbon Nanotubes, Nitrides and Silicon Carbide Diamond 2006. 40

[62] J. Sauerwald, D. Richter, E. Ansorge, B. Schmidt, and H. Fritze, "Doped monolithic langasite structures for high temperature MEMS," Solid State Ionics, vol. 179, no. 21-26, pp. 928-931, 2008. Solid State Ionics 16: Proceedings of the 16th International Conference on Solid State Ionics (SSI-16), Part I. 40

[63] D. Richter, S. Sakharov, E. Forsén, E. Mayer, L. Reindl, and H. Fritze, "Thin Film Electrodes for High Temperature Surface Acoustic Wave Devices," Proc. Eng., vol. 25, no. 0, pp. 168-171, 2011. EurosensorsXXV. 40

[64] H. Seh, H. L. Tuller, and H. Fritze, "Defect properties of langasite and effects on BAW gas sensor performance at high temperatures," J. of the Euro. Ceramic Soc., vol. 24, no. 6, pp. 1425-1429, 2004. Electroceramics VIII. 40

[65] J. Justice, L. Rodak, V. Narang, K. Lee, L. Hornak, and D. Korakakis, "Characterization of Group III-Nitride Based Surface Acoustic Wave Devices for High Temperature Applications," Proc. Mater. Res. Soc., vol. 1299, 1 2011. 41, 42, 44 
[66] J. A. Jahnke. Wiley, 2nd ed., April 2000. 44

[67] U. E. P. Agency, Gaseous Continuous Emission Monitoring Systems: Performance Specification Guidelines for SO2, NOx, CO2, O2 and TRS. BiblioGov, Feb 2013. 44

[68] N. Miura, K. Akisada, J. Wang, S. Zhuiykov, and T. Ono, "Mixed-potential-type NOx sensor based on YSZ and zinc oxide sensing electrode," Ionics, vol. 10, no. 1-2, pp. 1-9, 2004. 45

[69] H. Zheng and T. Guo, "Technical Study on Flue gas Continuous Emission Monitoring Systems for power plant," (Piscataway, NJ, USA), pp. 168-70, 2012//. 45

[70] B. Shi, Z. Ma, J. Jiang, and S. Duan, "Research on continuous emission monitoring system in thermal power plant," vol. 427-429, (Chongqing, China), pp. 1117-1120, 2013. 45

[71] L. Heyun, W. Feng, L. Yuping, Z. Weidong, and G. Chaochun, "Continuous emission monitoring system of power plant," Chin. Autom. Electr. Power Syst., vol. 25, no. 5, pp. 53-5, 2001/03/10. 45

[72] H. Zheng and G. Tang, "Developing data acquisition and handling system for continuous emission monitoring system from coal-fired power plant," (Piscataway, NJ, USA), pp. 3616-19, 2008/07/02. 45

[73] R. Roberson, "Continuous emission monitoring and quality assurance requirements for new power plants," vol. 1, (Philadelphia, PA, USA), pp. 13-15, 1988. 45

[74] J. Wang, B. Liao, X. Li, H. Ye, and Z. Yu, "A new continuous emission monitoring system by UV differential optical absorption spectroscopy," No. 529 CP, (Dublin, Ireland), pp. 667-671, 2007. 45

[75] H. Zheng, "Experiment study of continuous emission monitoring system based on differential optical absorption spectroscopy," vol. 1, (Shanghai, China), pp. 175177, 2009. 45

[76] Z. Xue-dian and X. Ke-xin, "Design a flue continuous emission monitoring system based on differential optical absorption spectroscopy," Chin. J. Sens. Actuators, vol. 20, no. 9, pp. 1963-6, Sept. 2007. 45

[77] C. Kamme, S. Viswanathan, and D. Sands, "A novel continuous emission monitoring system based on differential optical absorption spectroscopy," (Chicago, IL, USA), pp. $710-16,1992 / / .45$

[78] J. Justice, M. Elbaz, L. E. Rodak, and D. Korakakis, "High Harmonic Surface Acoustic Wave Devices for Harsh Environment Sensor Applications," Proc. Mater. Res. Soc., vol. 1519, 1 2013. 49 
[79] R. Dingle, H. L. Störmer, A. C. Gossard, and W. Wiegmann, "Electron mobilities in modulationdoped semiconductor heterojunction superlattices," Appl. Phys. Lett., vol. 33, no. 7, pp. 665-667, 1978. HEMT Reference 05. 53, 54

[80] L. Pfeiffer, K. W. West, H. L. Stormer, and K. W. Baldwin, "Electron mobilities exceeding $107 \mathrm{~cm} 2 / \mathrm{V}$ s in modulationdoped GaAs," Appl. Phys. Lett., vol. 55, no. 18, pp. 1888-1890, 1989. HEMT Reference 01. 53, 54

[81] K. B. Wong, J. P. Hagon, and M. Jaros, "The effects of modulation doping, static electric field and alloy ordering upon the electronic structure of GaAs-GaAlAs superlattices and heterojunctions," Semicon. Sci. and Techol., vol. 1, no. 43, p. 256, 1986. HEMT Reference 02. 53, 54, 55

[82] I. Buyanova, W. Chen, W. Bi, Y. Zeng, and C. Tu, "Intrinsic modulation doping in InP-based structures: properties relevant to device applications," J. Cryst. Growth, vol. 201-202, no. 0, pp. 786-789, 1999. HEMT Reference 03. 53

[83] H. Morkoç, R. Cingolani, and B. Gil, "Polarization effects in nitride semiconductor device structures and performance of modulation doped field effect transistors," Solid-State Electron., vol. 43, no. 10, pp. 1909-1927, 1999. HEMT Reference 06. 54

[84] G. Bastard, Wave Mechanics Applied to Semiconductor Heterostructures. WileyInterscience, Jan 1991. HEMT Reference 07. 54

[85] R. Oberhuber, G. Zandler, and P. Vogl, "Mobility of two-dimensional electrons in AlGaN/GaN modulation-doped field-effect transistors," Appl. Phys. Lett., vol. 73, no. 6, pp. 818-820, 1998. HEMT Reference 08. 54

[86] A. D. Carlo, S. Pescetelli, M. Paciotti, P. Lugli, and M. Graf, "Self-consistent tightbinding calculations of electronic and optical properties of semiconductor nanostructures," Solid State Commun., vol. 98, no. 9, pp. 803-806, 1996. HEMT Reference 09. 54

[87] F. Della Sala, A. Di Carlo, P. Lugli, F. Bernardini, V. Fiorentini, R. Scholz, and J. Jancu, "Free-carrier screening of polarization fields in wurtzite GaN/InGaN laser structures," Appl. Phys. Lett., vol. 74, no. 14, pp. 2002-2004, 1999. HEMT Reference 10. 54

[88] R. Cingolani, A. Botchkarev, H. Tang, H. Morkoç, G. Traetta, G. Coli, M. Lomascolo, A. Di Carlo, F. Della Sala, and P. Lugli, "Spontaneous polarization and piezoelectric field in $\mathrm{GaN} / \mathrm{Al}_{0.15} \mathrm{Ga}_{0.85} \mathrm{~N}$ quantum wells: Impact on the optical spectra," Phys. Rev. B, vol. 61, pp. 2711-2715, Jan 2000. HEMT Reference 11. 54

[89] H. Morkoç, A. D. Carlo, and R. Cingolani, "GaN-based modulation doped FETs and UV detectors," Solid-State Electron., vol. 46, no. 2, pp. 157-202, 2002. HEMT Reference 04. 55, 56 
[90] B. Monemar and G. Pozina, "Group III-nitride based hetero and quantum structures," Prog. Quan. Electron., vol. 24, no. 6, pp. 239-290, 2000. HEMT Reference 13. 55

[91] I. M. Watson, "Metal organic vapour phase epitaxy of AlN, GaN, InN and their alloys: A key chemical technology for advanced device applications," Coord. Chem. Rev., vol. 257, no. 13-14, pp. 2120-2141, 2013. HEMT Reference 14. 55

[92] O. Ambacher, J. Smart, J. R. Shealy, N. G. Weimann, K. Chu, M. Murphy, W. J. Schaff, L. F. Eastman, R. Dimitrov, L. Wittmer, M. Stutzmann, W. Rieger, and J. Hilsenbeck, "Two-dimensional electron gases induced by spontaneous and piezoelectric polarization charges in N- and Ga-face AlGaN/GaN heterostructures," J. of Appl. Phys., vol. 85, no. 6, pp. 3222-3233, 1999. HEMT Reference 16. 57, 58 